TRANSACTIONS OF THE

AMERICAN MATHEMATICAL SOCIETY

Volume 358 , Number 4 , Pages 1469-1510

S 0002-9947(05)03660-3

Article electronically published on March 25, 2005

\title{
ATOMIC AND MOLECULAR DECOMPOSITIONS OF ANISOTROPIC TRIEBEL-LIZORKIN SPACES
}

\author{
MARCIN BOWNIK AND KWOK-PUN HO
}

\begin{abstract}
Weighted anisotropic Triebel-Lizorkin spaces are introduced and studied with the use of discrete wavelet transforms. This study extends the isotropic methods of dyadic $\varphi$-transforms of Frazier and Jawerth $(1985,1989)$ to non-isotropic settings associated with general expansive matrix dilations and $A_{\infty}$ weights.

In close analogy with the isotropic theory, we show that weighted anisotropic Triebel-Lizorkin spaces are characterized by the magnitude of the $\varphi$-transforms in appropriate sequence spaces. We also introduce non-isotropic analogues of the class of almost diagonal operators and we obtain atomic and molecular decompositions of these spaces, thus extending isotropic results of Frazier and Jawerth.
\end{abstract}

\section{INTRODUCTION AND STATEMENTS OF MAIN RESULTS}

Many function spaces arising in harmonic analysis admit decompositions into simpler building blocks, often called atoms or molecules, that have some additional desirable properties. Perhaps the best known is the atomic decomposition of the Hardy spaces $H^{p}\left(\mathbb{R}^{n}\right), 0<p \leq 1$, which was first shown by Coifman [17] and was later extended to many other directions by a number of authors [19, 24, 29, 38.

One of the possible directions, where decomposition techniques are very useful, is the study of a large class of general Triebel-Lizorkin spaces $\dot{\mathbf{F}}_{p}^{\alpha, q}$ (homogeneous) and $\mathbf{F}_{p}^{\alpha, q}$ (inhomogeneous), $\alpha \in \mathbb{R}, 0<p, q \leq \infty$, which includes many well-known classical function spaces. In particular, $L^{p} \approx \dot{\mathbf{F}}_{p}^{0,2} \approx \mathbf{F}_{p}^{0,2}$ when $1<p<\infty$ and $H^{p} \approx \dot{\mathbf{F}}_{p}^{0,2}$ when $0<p \leq 1$. The atomic and molecular decomposition results for isotropic Triebel-Lizorkin spaces were first obtained by Frazier and Jawerth [27] with the help of the $\varphi$-transforms, which are now more often called discrete wavelet transforms.

The other possible direction of extending classical function spaces arising in harmonic analysis is the study of Euclidean spaces equipped with non-isotropic dilation structures. One of the first studies of this sort was accomplished by Calderón and

Received by the editors April 16, 2003 and, in revised form, March 8, 2004.

2000 Mathematics Subject Classification. Primary 42B25, 42B35, 42C40; Secondary 46E35, 47B37, 47B38.

Key words and phrases. Anisotropic Triebel-Lizorkin space, smooth atomic decomposition, smooth molecular decomposition, almost diagonal operators, wavelets, $\varphi$-transform, discrete wavelet transform.

The first author was partially supported by NSF grant DMS-0200080.

The authors thank Michael Frazier for careful reading and several suggestions for improvement of the paper, and Guido Weiss for making this joint work possible.

(C)2005 American Mathematical Society 
Torchinsky [14, 15] who introduced and studied parabolic Hardy spaces associated with certain one parameter groups of dilations on $\mathbb{R}^{n}$. Folland and Stein [24] extended this study to Hardy spaces defined on a class of homogeneous groups, which includes an important example of the Heisenberg group, where non-isotropic characteristics come naturally due to non-commutativity of a group action.

The non-isotropic variants of Triebel-Lizorkin and Besov spaces have been studied by Besov, Il'in, and Nikol'skiu [4 and Schmeisser and Triebel 39, 42. These studies were concerned mostly with Euclidean spaces associated with one-parameter groups of diagonal dilations of the form

$$
\left(\begin{array}{cccc}
2^{t a_{1}} & 0 & \cdots & 0 \\
0 & 2^{t a_{2}} & \cdots & 0 \\
\ldots \ldots & \ldots & \ldots & \ldots \\
0 & \cdots & 0 & 2^{t a_{n}}
\end{array}\right)
$$

where $t \in \mathbb{R}$ and $a=\left(a_{1}, \ldots, a_{n}\right)$ is a given anisotropy. More recently, Farkas 21] obtained atomic decomposition for Besov and Triebel-Lizorkin spaces associated with the above anisotropy. Furthermore, Berkolalko and Novikov [2, 3] constructed interesting Meyer-type wavelets adapted to any given anisotropy $a=\left(a_{1}, \ldots, a_{n}\right)$ and showed that the constructed wavelets form unconditional bases for the corresponding classes of Besov and Triebel-Lizorkin spaces.

Finally, there is also significant interest in the study of weighted function spaces associated with general $A_{\infty}$ weights. This direction of research was carried over by Bui, Paluszyński, and Taibleson [8, 9, 11, 12, for Besov and Triebel-Lizorkin spaces. The weighted Hardy spaces were studied by Strömberg and Torchinsky [40].

The goal of this work is to combine these developments into one coherent theory of weighted anisotropic Triebel-Lizorkin spaces on Euclidean spaces with the use of the discrete $\varphi$-transforms of Frazier and Jawerth. The main novelty of our approach is that we allow a fairly general discrete group of dilations, motivated by their role in the multidimensional theory of wavelets. More precisely, we introduce and study Triebel-Lizorkin spaces associated with an expansive dilation $A$, that is, an $n \times n$ real matrix all of whose eigenvalues $\lambda$ satisfy $|\lambda|>1$. Our formulation includes the previously-studied classes of Triebel-Lizorkin spaces that corresponded to diagonal dilations. In what follows we summarize the results obtained in this work.

The starting point in the theory of discrete $\varphi$-transforms of Frazier and Jawerth is a basic representation formula for tempered distributions $f=\sum_{Q}\left\langle f, \varphi_{Q}\right\rangle \psi_{Q}$, where the sum runs over all dyadic cubes $Q$ in $\mathbb{R}^{n}$, and $\varphi_{Q}$ and $\psi_{Q}$ are translates and dilates of $\varphi$ and $\psi$ localized to a dyadic cube $Q$. Lemma 2.8 generalizes this formula to a non-isotropic setting, where the dyadic cubes $Q$ are replaced by a collection of dilated cubes

$$
\mathcal{Q}=\left\{Q=A^{j}\left([0,1]^{n}+k\right): j \in \mathbb{Z}, k \in \mathbb{Z}^{n}\right\}
$$

adapted to the action of a dilation $A$. Naturally, the functions $\varphi$ and $\psi$ have to satisfy support conditions (2.9) and the Calderón reproducing formula (2.10). In particular, $\hat{\varphi}$ and $\hat{\psi}$ have to be smooth and compactly supported.

Following Frazier and Jawerth, we then define the $\varphi$-transform, which maps the distribution $f$ to the sequence of its wavelet coefficients $S_{\varphi} f=\left\{\left\langle f, \varphi_{Q}\right\rangle\right\}_{Q \in \mathcal{Q}}$. For any sequence $s=\left\{s_{Q}\right\}_{Q \in \mathcal{Q}}$ of complex numbers, we formally define the inverse $\varphi$-transform, which maps $s$ to a distribution $T_{\psi} s=\sum_{Q \in \mathcal{Q}} s_{Q} \psi_{Q}$. To guarantee 
meaningfulness and boundedness of these transforms, we need to introduce quantitative assumptions on distributions $f$ and sequences $s$. We will assume that $f$ belongs to anisotropic Triebel-Lizorkin space $\dot{\mathbf{F}}_{p}^{\alpha, q}$ and $s$ belongs to its discrete variant $\dot{\mathbf{f}}_{p}^{\alpha, q}$.

Given $\alpha \in \mathbb{R}, 0<p<\infty, 0<q \leq \infty$, and a dilation $A$, we introduce the anisotropic Triebel-Lizorkin space $\dot{\mathbf{F}}_{p}^{\alpha, q}$ as the collection of all tempered distributions $f$ (modulo polynomials) such that

$$
\|f\|_{\dot{\mathbf{F}}_{p}^{\alpha, q}}=\left\|\left(\sum_{j \in \mathbb{Z}}\left(|\operatorname{det} A|^{j \alpha}\left|f * \varphi_{j}\right|\right)^{q}\right)^{1 / q}\right\|_{L^{p}}<\infty,
$$

where $\varphi \in \mathcal{S}\left(\mathbb{R}^{n}\right)$ satisfies certain support conditions (3.2) and (3.3), and $\varphi_{j}(x)=$ $|\operatorname{det} A|^{j} \varphi\left(A^{j} x\right)$. In Corollary 3.7 we show that this definition is independent of the choice of $\varphi$ in a more general weighted setting, where $L^{p}\left(\mathbb{R}^{n}\right)$ is replaced by $L^{p}\left(\mathbb{R}^{n}, w d x\right)$ with a weight $w$ in the Muckenhoupt $A_{\infty}$ class.

In the standard dyadic case $A=2 I d$ we have $\operatorname{det} A=2^{n}$ and the factor $|\operatorname{det} A|^{j \alpha}$ in the above definition would be $2^{j \alpha n}$ instead of the usual $2^{j \alpha}$. Thus, our convention amounts to rescaling the smoothness index $\alpha$, which in the traditional case is thought of as the number of derivatives.

The discrete Triebel-Lizorkin sequence space, $\dot{\mathbf{f}}_{p}^{\alpha, q}$, is defined as the collection of all complex-valued sequences $s=\left\{s_{Q}\right\}_{Q \in \mathcal{Q}}$ such that

$$
\|s\|_{\dot{\mathbf{f}}_{p}^{\alpha, q}}=\left\|\left(\sum_{Q \in \mathcal{Q}}\left(|Q|^{-\alpha}\left|s_{Q}\right| \tilde{\chi}_{Q}\right)^{q}\right)^{1 / q}\right\|_{L^{p}}<\infty
$$

where $\tilde{\chi}_{Q}=|Q|^{-1 / 2} \chi_{Q}$ is the $L^{2}$-normalized characteristic function of the dilated cube $Q$.

Our basic result, Theorem 3.5 is the following generalization of the fundamental result of Frazier and Jawerth [27, Theorem 2.2].

Theorem 1.1. The $\varphi$-transform $S_{\varphi}: \dot{\mathbf{F}}_{p}^{\alpha, q} \rightarrow \dot{\mathbf{f}}_{p}^{\alpha, q}$ and the inverse $\varphi$-transform $T_{\psi}: \dot{\mathbf{f}}_{p}^{\alpha, q} \rightarrow \dot{\mathbf{F}}_{p}^{\alpha, q}$ are bounded, and $T_{\psi} \circ S_{\varphi}$ is the identity on $\dot{\mathbf{F}}_{p}^{\alpha, q}$.

Theorem 1.1 can then be exploited to obtain applications for the $\dot{\mathbf{F}}_{p}^{\alpha, q}$ spaces by proving corresponding assertions for $\dot{\mathbf{f}}_{p}^{\alpha, q}$ as it was done in [27]. This is because the $\dot{\mathbf{f}}_{p}^{\alpha, q}$ norm is generally easier to work with, since it is discrete and depends only on the magnitude of the sequence elements. Therefore, following the approach of Frazier and Jawerth, we will study operators on $\dot{\mathbf{F}}_{p}^{\alpha, q}$ by considering corresponding operators on $\dot{\mathbf{f}}_{p}^{\alpha, q}$. One of the most useful sufficient conditions for the boundedness of operators on $\dot{\mathbf{f}}_{p}^{\alpha, q}$ is the almost diagonal condition studied in great detail in [27]. We extend this notion to a non-isotropic setting and we show that the expected boundedness result, Theorem 4.1, holds for anisotropic Triebel-Lizorkin spaces.

In Section 5 we introduce the notion of smooth molecules for anisotropic TriebelLizorkin spaces extending familiar isotropic molecules introduced in [27. A smooth molecule supported near the dilated cube $Q \in \mathcal{Q}$ must satisfy appropriate smoothness, decay, and vanishing moments properties. In Theorem5.5, we establish the estimate $\left\|\sum_{Q} s_{Q} \Psi_{Q}\right\|_{\dot{\mathbf{F}}_{p}^{\alpha, q}} \leq C\|s\|_{\dot{\mathbf{f}}_{p}^{\alpha, q}}$, where $\left\{\Psi_{Q}\right\}_{Q}$ are smooth synthesis molecules. Likewise, in Theorem 5.6. we show $\left\|\left\{\left\langle f, \Phi_{Q}\right\rangle\right\}_{Q}\right\|_{\dot{\mathbf{f}}_{p}^{\alpha, q}} \leq C\|f\|_{\dot{\mathbf{F}}_{p}^{\alpha, q}}$, where $\left\{\Phi_{Q}\right\}_{Q}$ are smooth analysis molecules. Both of these results generalize the boundedness of the 
$\varphi$-transform and the inverse $\varphi$-transform in Theorem 1.1 to situations when neither $\left\{\Phi_{Q}\right\}_{Q}$ nor $\left\{\Psi_{Q}\right\}_{Q}$ are necessarily obtained by translates and dilates of a particular function in $\mathcal{S}$. These results are then used to show the smooth atomic decomposition of the anisotropic $\dot{\mathbf{F}}_{p}^{\alpha, q}$ spaces. In Theorem 5.8 we show that every $f \in \dot{\mathbf{F}}_{p}^{\alpha, q}$ can be decomposed as $f=\sum_{Q} s_{Q} a_{Q}$ with $\left\|\left\{s_{Q}\right\}_{Q}\right\|_{\dot{\mathbf{f}}_{p}^{\alpha, q}} \leq C\|f\|_{\dot{\mathbf{F}}_{p}^{\alpha, q}}$, where $a_{Q}$ 's are smooth atoms. That is, each $a_{Q}$ is compactly supported near the dilated cube $Q$ and satisfies appropriate smoothness and vanishing moments conditions. This result is again an extension of 27] to the weighted anisotropic Triebel-Lizorkin spaces.

Finally, we also study inhomogeneous anisotropic Triebel-Lizorkin spaces $\mathbf{F}_{p}^{\alpha, q}$ and we outline analogous decomposition results for these spaces. Section 6 includes the proofs of some of more technical and longer auxiliary results needed in this work.

\section{Some BACKGround TOOLS}

We start by recalling basic definitions and properties of non-isotropic spaces associated with general expansive dilations.

2.1. Basic facts about expansive dilations and $A_{p}$ weights. A real $n \times n$ matrix $A$ is an expansive matrix, sometimes called shortly a dilation, if $\min _{\lambda \in \sigma(A)}|\lambda|>1$, where $\sigma(A)$ is the set of all eigenvalues (the spectrum) of $A$. A basic notion in our study is a quasi-norm $\rho_{A}$ associated with $A$, which induces a quasi-distance making $\mathbb{R}^{n}$ a space of homogeneous type. For rudimentary facts about spaces of homogeneous type we refer the reader to [18, 19, 29].

Definition 2.1. A quasi-norm associated with an expansive matrix $A$ is a measurable mapping $\rho_{A}: \mathbb{R}^{n} \rightarrow[0, \infty)$ satisfying

$$
\begin{aligned}
\rho_{A}(x) & >0, & & \text { for } x \neq 0, \\
\rho_{A}(A x) & =|\operatorname{det} A| \rho_{A}(x) & & \text { for } x \in \mathbb{R}^{n}, \\
\rho_{A}(x+y) & \leq H\left(\rho_{A}(x)+\rho_{A}(y)\right) & & \text { for } x, y \in \mathbb{R}^{n},
\end{aligned}
$$

where $H \geq 1$ is a constant.

In the standard dyadic case $A=2 I d$, a quasi-norm $\rho_{A}$ satisfies $\rho_{A}(2 x)=2^{n} \rho_{A}(x)$ instead of the usual scalar homogeneity. In particular, $\rho_{A}(x)=|x|^{n}$ is an example for a quasi-norm for $A=2 I d$, where $|\cdot|$ represent the Euclidean norm in $\mathbb{R}^{n}$.

For a list of properties of quasi-norms associated with expansive dilations we refer the reader to 6, 32. Here, we only recall a few basic facts needed in this work. One can show that all quasi-norms associated to a fixed dilation $A$ are equivalent; see [6, Lemma 2.4]. Moreover, there always exists a quasi-norm $\rho_{A}$, which is $C^{\infty}$ on $\mathbb{R}^{n}$ except the origin; see [33. However, for our purposes it is enough to restrict to a quasi-norm $\rho_{A}$ given by

$$
\rho_{A}(x)=\sum_{k=-\infty}^{\infty}|\operatorname{det} A|^{k} \chi_{O_{k}}(x),
$$

where $O_{k}=A^{k}(B(0,1)) \backslash \bigcup_{j=-\infty}^{k-1} A^{j}(B(0,1))$, and $B(0,1)=\left\{x \in \mathbb{R}^{n}:|x|<1\right\}$ is the unit ball. Equivalently,

$$
\rho_{A}(x)=|\operatorname{det} A|^{k}, \quad \text { where } k=\inf \left\{j \in \mathbb{Z}: A^{-j} x \in B(0,1)\right\}
$$


for $x \neq 0$ and $\rho_{A}(x)=0$ for $x=0$. It is then clear that $\rho_{A}$ given by (2.2) satisfies (2.1) with the constant $H=|\operatorname{det} A|^{j_{0}}$, where $j_{0}$ is the smallest integer such that $\bigcup_{j \leq 0} A^{j}(B(0,2)) \subset A^{j_{0}}(B(0,1))$. Moreover, one can show that the above quasinorm satisfies

$$
\left|\left\{x \in \mathbb{R}^{n}: \rho_{A}(x)<r\right\}\right| \approx r \quad \text { for any } r>0 .
$$

Since all quasi-norms associated to a fixed dilation $A$ are equivalent, (2.4) holds for any quasi-norm $\rho_{A}$ associated with $A$.

It should be remarked that the quasi-norm $\rho_{A}$ given by (2.2) might produce $\rho_{A}$-balls $\left\{x \in \mathbb{R}^{n}: \rho_{A}(x)<r\right\}$, which are not convex. Despite this, it is possible to modify the above construction to guarantee that $\rho_{A}$-balls are convex. To achieve this, one must replace the ball $B(0,1)$ in $(2.3)$ by an appropriate ellipsoid $\Delta$ satisfying $\Delta \subset r \Delta \subset A \Delta$ for some $r>1$. For more details, we refer to [6, p. 5]. Therefore, we will simply assume that $\rho_{A}$-balls are convex.

We also need the following basic facts about the quasi-norm $\rho_{A}$; see [6, 33].

Proposition 2.1. For any expansive matrix A, there exists a constant $c>0$ such that $\left|O_{k}\right|=c|\operatorname{det} A|^{k}$ for any $k \in \mathbb{Z}$. Consequently, for any $\epsilon>0$,

$$
\int_{B(0,1)} \rho_{A}(x)^{\epsilon-1} d x<\infty \quad \text { and } \quad \int_{\mathbb{R}^{n} \backslash B(0,1)} \rho_{A}(x)^{-1-\epsilon} d x<\infty .
$$

Lemma 2.2. Suppose $A$ is expansive matrix, and $\lambda_{-}$and $\lambda_{+}$are any positive real numbers such that $1<\lambda_{-}<\min _{\lambda \in \sigma(A)}|\lambda|$ and $\lambda_{+}>\max _{\lambda \in \sigma(A)}|\lambda|$. Let

$$
\zeta_{+}:=\frac{\ln \lambda_{+}}{\ln |\operatorname{det} A|}, \quad \zeta_{-}:=\frac{\ln \lambda_{-}}{\ln |\operatorname{det} A|} .
$$

Then for any quasi-norm $\rho_{A}$ there exists a constant $C$ such that

$$
C^{-1} \rho_{A}(x)^{\zeta_{-}} \leq|x| \leq C \rho_{A}(x)^{\zeta_{+}} \quad \text { if } \quad \rho_{A}(x) \geq 1
$$

and

$$
C^{-1} \rho_{A}(x)^{\zeta_{+}} \leq|x| \leq C \rho_{A}(x)^{\zeta_{-}} \quad \text { if } \quad \rho_{A}(x) \leq 1 .
$$

Furthermore, if $A$ is diagonalizable over $\mathbb{C}$, then we may take $\lambda_{-}=\min _{\lambda \in \sigma(A)}|\lambda|$ and $\lambda_{+}=\max _{\lambda \in \sigma(A)}|\lambda|$.

We will also need the following easy estimates

$$
\begin{array}{ll}
(1 / c) \lambda_{-}^{j}|x| \leq\left|A^{j} x\right| \leq c \lambda_{+}^{j}|x| & \text { for } j \geq 0, \\
(1 / c) \lambda_{+}^{j}|x| \leq\left|A^{j} x\right| \leq c \lambda_{-}^{j}|x| & \text { for } j \leq 0,
\end{array}
$$

for some constant $c>0$, where $\lambda_{-}$and $\lambda_{+}$are the same as in Lemma 2.2, see [41, p. 40]. It is also easy to verify that we have the following proposition.

Proposition 2.3. $\left(\mathbb{R}^{n}, \rho_{A},|\cdot|\right)$ is a space of homogeneous type, where $\rho_{A}$ is a quasi-norm associated with an expansive dilation $A$, and $|\cdot|$ is Lebesgue measure on $\mathbb{R}^{n}$.

We also need some basic results about Muckenhoupt $A_{p}$ weights on spaces of homogeneous type. The main tool needed in this work is the weighted vectorvalued Fefferman-Stein inequality for the Hardy-Littlewood maximal operator. For basic facts about weighted norm inequalities, we refer to [29, 38. 
Definition 2.2. We say that a function $w: \mathbb{R}^{n} \rightarrow(0, \infty)$ belongs to the Muckenhoupt class $A_{p}=A_{p}\left(\mathbb{R}^{n}, \rho_{A}\right), p>1$, if there is a constant $C>0$ such that

$$
\left(\frac{1}{|B|} \int_{B} w(y) d y\right)\left(\frac{1}{|B|} \int_{B} w^{-\frac{1}{(p-1)}}(y) d y\right)^{p-1} \leq C,
$$

for any $\rho_{A}$-ball $B \in \mathcal{B}$. Here, $\mathcal{B}=\mathcal{B}\left(\rho_{A}\right)$ is the collection of all $\rho_{A}$-balls

$$
B_{\rho_{A}}(x, r)=\left\{y \in \mathbb{R}^{n}: \rho_{A}(y-x)<r\right\} \quad x \in \mathbb{R}^{n}, r>0 .
$$

For $p=1$, we say $w \in A_{1}$ if

$$
\left(\frac{1}{|B|} \int_{B} w(y) d y\right)\left(\sup _{B} w^{-1}\right) \leq C,
$$

for any $\rho_{A}$-ball $B \in \mathcal{B}$. Finally, $A_{\infty}=\bigcup_{p>1} A_{p}$.

For any locally integrable function $f$ on $\mathbb{R}^{n}$, we define the Hardy-Littlewood maximal operator $M_{\rho_{A}}$ to be

$$
M_{\rho_{A}} f(x)=\sup _{x \in B \in \mathcal{B}} \frac{1}{|B|} \int_{B}|f(y)| d y,
$$

where $\mathcal{B}$ is the collection of all $\rho_{A}$-balls $B$.

We will use the following two standard results on weighted norm inequalities.

Theorem 2.4. The Hardy-Littlewood maximal operator $M_{\rho_{A}}$ is of weak type $(1,1)$ on $L^{1}(w)$ if and only if $w \in A_{1}$ and $M_{\rho_{A}}$ is bounded on $L^{p}(w)$ for $1<p<\infty$ if and only if $w \in A_{p}$.

Theorem 2.4 is an immediate consequence of Proposition2.3 and standard results on weighted norm inequalities; see [29, Chapter IV] or [38, Chapter V]. In fact, Theorem 2.4 is a special case of a more general Theorem 2.5 which is the FeffermanStein vector-valued inequality [22] in the weighted setting.

Theorem 2.5. Suppose that $1<p<\infty, 1<q \leq \infty$, and $w \in A_{p}$. Then there exists a constant $C$ such that

$$
\left\|\left(\sum_{i}\left|M_{\rho_{A}} f_{i}\right|^{q}\right)^{1 / q}\right\|_{L^{p}(w)} \leq C\left\|\left(\sum_{i}\left|f_{i}\right|^{q}\right)^{1 / q}\right\|_{L^{p}(w)}
$$

holds for any $\left(f_{i}\right)_{i} \subset L^{p}(w)$.

For a direct proof of this result for $\mathbb{R}^{n}$ with the usual isotropic distance metric we refer to [1; see also [29, Remark V.6.5]. By Proposition 2.3 and [30, Section $6.6]$, Theorem 2.5 also holds for $\mathbb{R}^{n}$ in the anisotropic setting.

2.2. Discrete wavelet transforms. Suppose that $\varphi, \psi$ are test functions in the Schwartz class $\mathcal{S}\left(\mathbb{R}^{n}\right)$ such that

$$
\begin{gathered}
\operatorname{supp} \hat{\varphi}, \operatorname{supp} \hat{\psi} \subset[-\pi, \pi]^{n} \backslash\{0\}, \\
\sum_{j \in \mathbb{Z}} \frac{\hat{\varphi}\left(\left(A^{*}\right)^{j} \xi\right)}{\psi} \hat{\psi}\left(\left(A^{*}\right)^{j} \xi\right)=1 \quad \text { for all } \xi \in \mathbb{R}^{n} \backslash\{0\},
\end{gathered}
$$

where $A^{*}$ is the adjoint (transpose) of $A$. Here,

$$
\operatorname{supp} \hat{\varphi}=\overline{\left\{\xi \in \mathbb{R}^{n}: \hat{\varphi}(\xi) \neq 0\right\}},
$$


and the Fourier transform of $f$ is

$$
\hat{f}(\xi)=\int_{\mathbb{R}^{n}} f(x) e^{-i\langle x, \xi\rangle} d x .
$$

For any $j \in \mathbb{Z}$ and $k \in \mathbb{Z}^{n}$, let $Q_{j, k}=A^{-j}\left([0,1]^{n}+k\right)$ be the dilated cube, and $x_{Q_{j, k}}=A^{-j} k$ be its "lower-left corner". Let

$$
\mathcal{Q}=\mathcal{Q}_{A}=\left\{Q_{j, k}: j \in \mathbb{Z}, k \in \mathbb{Z}^{n}\right\}
$$

be the collection of all dilated cubes. For $\varphi \in \mathcal{S}\left(\mathbb{R}^{n}\right)$, define

$$
\begin{aligned}
& \varphi_{j}(x)=|\operatorname{det} A|^{j} \varphi\left(A^{j} x\right) \quad \text { for } j \in \mathbb{Z}, \\
& \varphi_{Q}(x)=|\operatorname{det} A|^{j / 2} \varphi\left(A^{j} x-k\right)=|Q|^{1 / 2} \varphi_{j}\left(x-x_{Q}\right) \quad \text { for } Q=Q_{j, k} \in \mathcal{Q} .
\end{aligned}
$$

It is not hard to show that the conditions (2.9), (2.10) imply that the wavelet systems $\left\{\varphi_{Q}: Q \in \mathcal{Q}\right\}$ and $\left\{\psi_{Q}: Q \in \mathcal{Q}\right\}$ form a pair of dual frames in $L^{2}\left(\mathbb{R}^{n}\right)$. This means that $\left\{\varphi_{Q}: Q \in \mathcal{Q}\right\}$ and $\left\{\psi_{Q}: Q \in \mathcal{Q}\right\}$ are Bessel sequences, i.e., there exists a constant $C>0$ such that

$$
\sum_{Q \in \mathcal{Q}}\left|\left\langle f, \varphi_{Q}\right\rangle\right|^{2}, \sum_{Q \in \mathcal{Q}}\left|\left\langle f, \psi_{Q}\right\rangle\right|^{2} \leq C|| f \|_{L^{2}}^{2} \quad \text { for all } f \in L^{2}\left(\mathbb{R}^{n}\right),
$$

and

$$
f=\sum_{Q \in \mathcal{Q}}\left\langle f, \varphi_{Q}\right\rangle \psi_{Q} \quad \text { for all } f \in L^{2}\left(\mathbb{R}^{n}\right),
$$

where the above series converges unconditionally in $L^{2}$. Indeed, using (2.9) and a standard periodization argument (see for example [5, Lemma 3.1]), it is not hard to show that

$$
\sum_{Q \in \mathcal{Q}}\left|\left\langle f, \varphi_{Q}\right\rangle\right|^{2}=\sum_{j \in \mathbb{Z}}|\hat{f}(\xi)|^{2}\left|\hat{\varphi}\left(\left(A^{*}\right)^{j} \xi\right)\right|^{2} d \xi \quad \text { for all } f \in L^{2}\left(\mathbb{R}^{n}\right),
$$

and the similar identity for $\psi$. This together with (2.9) shows (2.11). Applying analogous periodization arguments such as [5, Theorem 3.2 and Theorem 4.2] and using (2.10) yields (2.12). We remark that (2.12) also follows from more general considerations in [16].

Since our interest lies beyond $L^{2}$ theory of wavelet decompositions, we will need the following two lemmas providing basic reproducing identities (2.14) and (2.23) used subsequently in the study of $\varphi$ transform. Lemma 2.6 shows that any distribution $f$ admits a kind of Littlewood-Paley decomposition adapted to an expansive dilation $A$, whereas Lemma 2.8 provides the fundamental reproducing identity for distributions by means of discrete wavelet transforms. Both of these results are anisotropic modifications of their well-known dyadic analogues; see [25, 27, 28.

Lemma 2.6. Suppose that $A$ is an expansive matrix and $\varphi \in \mathcal{S}\left(\mathbb{R}^{n}\right)$ is such that

$$
\sum_{j \in \mathbb{Z}} \hat{\varphi}\left(\left(A^{*}\right)^{j} \xi\right)=1 \quad \text { for all } \xi \in \mathbb{R}^{n} \backslash\{0\},
$$

and $\operatorname{supp} \hat{\varphi}$ is compact and bounded away from the origin. Then for any $f \in \mathcal{S}^{\prime}\left(\mathbb{R}^{n}\right)$,

$$
f=\sum_{j \in \mathbb{Z}} \varphi_{j} * f
$$

where $\varphi_{j}(x)=|\operatorname{det} A|^{j} \varphi\left(A^{j} x\right)$, and the convergence is in $\mathcal{S}^{\prime} / \mathcal{P}$, where $\mathcal{P} \subset \mathcal{S}^{\prime}$ is the class of all polynomials in $\mathbb{R}^{n}$. 
More precisely, there exist a constant d depending only on the order of the distribution $\hat{f}$, a sequence of polynomials $\left\{P_{k}\right\}_{k=1}^{\infty} \subset \mathcal{P}$ with $\operatorname{deg} P_{k} \leq d$, and $P \in \mathcal{P}$, such that

$$
f=\lim _{k \rightarrow \infty}\left(\sum_{j=-k}^{\infty} \varphi_{j} * f+P_{k}\right)+P
$$

where the convergence is in $\mathcal{S}^{\prime}$.

To show Lemma 2.6 we will need to use the following proposition, which is of independent interest.

Proposition 2.7. Suppose $\left\{f_{i}\right\}_{i \in \mathbb{N}}$ is a sequence of distributions in $\mathcal{S}^{\prime}\left(\mathbb{R}^{n}\right)$ and $d \geq 0$ is an integer. Assume that for every multi-index $\gamma$ with $|\gamma|=d+1$ the sequence of partial derivatives $\left\{\partial^{\gamma} f_{i}\right\}$ converges in $\mathcal{S}^{\prime}$ as $i \rightarrow \infty$. Then there exists a sequence of polynomials $\left\{P_{i}\right\}_{i \in \mathbb{N}}$ with $\operatorname{deg} P_{i} \leq d$ such that $\left\{f_{i}+P_{i}\right\}$ converges to some distribution $f \in \mathcal{S}^{\prime}$ as $i \rightarrow \infty$.

Proposition 2.7 is probably a folklore fact; see [36, p. 53]. Since we could not find its proof in the literature, we include the proof of Proposition 2.7 in Section 6 ,

Proof of Lemma 2.6. Take any $f \in \mathcal{S}^{\prime}$ and suppose that $\hat{f}$ has order $\leq m$. This means that there exists an integer $l \geq 0$ and a constant $C$ such that

$$
|\langle\hat{f}, \phi\rangle| \leq C \sup _{|\alpha| \leq l,|\beta| \leq m}\|\phi\|_{\alpha, \beta} \quad \text { for all } \phi \in \mathcal{S},
$$

where $\|\phi\|_{\alpha, \beta}=\sup _{x \in \mathbb{R}^{n}}\left|x^{\alpha} \| \partial^{\beta} \phi(x)\right|$ denotes the usual semi-norm in $\mathcal{S}\left(\mathbb{R}^{n}\right)$ for multi-indices $\alpha$ and $\beta$. Suppose that $\varphi \in \mathcal{S}$ satisfies the hypothesis of Lemma 2.6. In particular, there exists a constant $c>0$ such that

$$
\operatorname{supp} \hat{\varphi} \subset\left\{\xi \in \mathbb{R}^{n}: 1 / c<|\xi|<c\right\} .
$$

We will first show that the series $\sum_{j \geq 0} \varphi_{j} * f$ converges in $\mathcal{S}^{\prime}$. Since the Fourier transform is an isomorphism of $\mathcal{S}^{\prime}$, this is equivalent to saying that $\sum_{j \geq 0} \widehat{\varphi_{j}} \hat{f}$ converges in $\mathcal{S}^{\prime}$. By (2.16)

$$
\left|\left\langle\widehat{\varphi_{j}} \hat{f}, \phi\right\rangle\right|=\left|\left\langle\hat{f}, \widehat{\varphi_{j}} \phi\right\rangle\right| \leq C \sup _{|\alpha| \leq l,|\beta| \leq m}\left\|\widehat{\varphi_{j}} \phi\right\|_{\alpha, \beta} .
$$

Since $\widehat{\varphi_{j}}(\xi)=\hat{\varphi}\left(\left(A^{*}\right)^{-j} \xi\right)$, by applying the chain rule we have

$$
\sup _{|\beta|=s}\left\|\partial^{\beta} \widehat{\varphi_{j}}\right\|_{\infty} \leq C\left\|\left(A^{*}\right)^{-j}\right\|^{s} \sup _{|\beta|=s}\left\|\partial^{\beta} \hat{\varphi}\right\|_{\infty}
$$

Since the norms $\left\|\left(A^{*}\right)^{-j}\right\|$ are uniformly bounded for all $j \geq 0$ by the expansiveness of $A^{*}$, we have by (2.17)

$$
\begin{aligned}
& \sup _{|\alpha| \leq l,|\beta| \leq m}\left\|\widehat{\varphi_{j}} \phi \mid\right\|_{\alpha, \beta} \leq C \sup _{\xi \in \mathbb{R}^{n}}\left((1+|\xi|)^{l} \sup _{|\beta| \leq m}\left|\partial^{\beta} \widehat{\varphi_{j}}(\xi)\right| \cdot \sup _{|\beta| \leq m}\left|\partial^{\beta} \phi(\xi)\right|\right) \\
& \leq C \sup _{1 / c<\left|\left(A^{*}\right)^{-j} \xi\right|<c}(1+|\xi|)^{l} \sup _{|\beta| \leq m}\left|\partial^{\beta} \phi(\xi)\right| \\
& \leq C \sup _{|\alpha| \leq l+1,|\beta| \leq m}\|\phi\|_{\alpha, \beta} \sup _{1 / c<\left|\left(A^{*}\right)^{-j} \xi\right|<c}(1+|\xi|)^{-1} \\
& \leq C \sup _{|\alpha| \leq l+1,|\beta| \leq m}\|\phi\|_{\alpha, \beta} \sup _{|\xi|>1 / c}\left(1+\left|\left(A^{*}\right)^{j} \xi\right|\right)^{-1} \leq C \lambda_{-}^{-j} \sup _{|\alpha| \leq l+1,|\beta| \leq m}\|\phi\|_{\alpha, \beta}
\end{aligned}
$$


where in the last step we used (2.7) with $\lambda_{-}$being the same as in Lemma 2.2. Since $A^{*}$ is expansive, we can choose $\lambda_{-}>1$. Combining (2.18) and (2.20) shows that $\sum_{j \geq 0} \widehat{\varphi_{j}} \hat{f}$ converges in $\mathcal{S}^{\prime}$.

Next we will show that for sufficiently large $d$, the series $\sum_{j<0} \partial^{\gamma}\left(\varphi_{j} * f\right)$ converges in $\mathcal{S}^{\prime}$ for every multi-index $|\gamma|=d+1$. Again, this is equivalent to saying that $\sum_{j<0} \xi^{\gamma} \widehat{\varphi_{j}} \hat{f}$ converges in $\mathcal{S}^{\prime}$ with $|\gamma|=d+1$.

Choose any integer $d \geq\left\lfloor m \ln \lambda_{+} / \ln \lambda_{-}\right\rfloor$, where $\lambda_{+}$and $\lambda_{-}$are the same as in Lemma 2.2. Repeating the estimates as in (2.20) we have by (2.19) for $j<0$

$$
\begin{aligned}
& \sup _{|\alpha| \leq l,|\beta| \leq m}\left\|\xi^{\gamma} \widehat{\varphi_{j}} \phi\right\|_{\alpha, \beta} \\
& \leq C \sup _{0 \leq k \leq d+1} \sup _{\xi \in \mathbb{R}^{n}}\left((1+|\xi|)^{l}|\xi|^{d+1-k} \sup _{|\beta| \leq m-k}\left|\partial^{\beta} \widehat{\varphi_{j}}(\xi)\right| \cdot \sup _{|\beta| \leq m-k}\left|\partial^{\beta} \phi(\xi)\right|\right) \\
& \leq C \sup _{0 \leq k \leq d+1}\left\|\left(A^{*}\right)^{-j}\right\|^{m-k} \sup _{|\beta| \leq m-k}\|\phi\|_{0, \beta} \sup _{1 / c<\left|\left(A^{*}\right)^{-j} \xi\right|<c}(1+|\xi|)^{l}|\xi|^{d+1-k} \\
& \leq C \sup _{0 \leq k \leq d+1}\left\|\left(A^{*}\right)^{-j}\right\|^{m-k} \sup _{|\beta| \leq m}\|\phi\|_{0, \beta} \sup _{|\xi|<c}\left(1+\left|\left(A^{*}\right)^{j} \xi\right|\right)^{l}\left|\left(A^{*}\right)^{j} \xi\right|^{d+1-k} \\
& \leq C \sup _{0 \leq k \leq d+1} \lambda_{+}^{-j(m-k)} \lambda_{-}^{j(d+1-k)} \sup _{|\beta| \leq m}\|\phi\|_{0, \beta} \leq C \lambda_{+}^{-j m} \lambda_{-}^{j(d+1)} \sup _{|\beta| \leq m}\|\phi\|_{0, \beta},
\end{aligned}
$$

where in the last two steps we used (2.8) and $\left(\lambda_{+} / \lambda_{-}\right)^{j}<1$ for $j<0$, respectively. To clarify (2.21), we note that $0 \leq k \leq d+1$ represents the number of derivatives that fall on the term $\xi^{\gamma}$ when applying the Leibniz rule for $\partial^{\beta}\left(\xi^{\gamma} \widehat{\varphi_{j}} \phi\right)$. Therefore, by (2.16) and (2.21) for any $|\gamma|=d+1$ we have

$$
\left|\left\langle\xi^{\gamma} \widehat{\varphi_{j}} \hat{f}, \phi\right\rangle\right|=\left|\left\langle\hat{f}, \xi^{\gamma} \widehat{\varphi_{j}} \phi\right\rangle\right| \leq C\left(\lambda_{+}^{-m} \lambda_{-}^{d+1}\right)^{j} \sup _{|\beta| \leq m}\|\phi\|_{0, \beta},
$$

which, by our choice of $d$, implies that $\sum_{j<0} \xi^{\gamma} \widehat{\varphi_{j}} \hat{f}$ converges in $\mathcal{S}^{\prime}$, since $\lambda_{+}^{-m} \lambda_{-}^{d+1}$ $>1$. Since $|\gamma|=d+1$ is arbitrary, Proposition 2.7 implies the existence of polynomials $\left\{P_{k}\right\}$, such that $\left\{\sum_{j=-k}^{-1} \varphi_{j} * f+P_{k}\right\}$ converges in $\mathcal{S}^{\prime}$ as $k \rightarrow \infty$.

Combining the above yields that $\left\{\sum_{j=-k}^{\infty} \varphi_{j} * f+P_{k}\right\}$ converges to some distribution $f_{0} \in \mathcal{S}^{\prime}$ as $k \rightarrow \infty$. By (2.13) it is clear that $\operatorname{supp}\left(\hat{f}-\hat{f}_{0}\right)=\{0\}$ by testing against $\phi \in \mathcal{S}$ with $0 \notin \operatorname{supp} \phi$. Therefore, there exists a polynomial $P$ such that $f=f_{0}+P$, which completes the proof of Lemma 2.6.

Lemma 2.8. Suppose that $A$ is an expansive matrix. If $g \in \mathcal{S}^{\prime}\left(\mathbb{R}^{n}\right), h \in \mathcal{S}\left(\mathbb{R}^{n}\right)$ and

$$
\operatorname{supp} \hat{g}, \hat{h} \subset\left(A^{*}\right)^{j}[-\pi, \pi]^{n} \quad \text { for some } j \in \mathbb{Z} \text {, }
$$

then

$$
(g * h)(x)=\sum_{k \in \mathbb{Z}^{n}}|\operatorname{det} A|^{-j} g\left(A^{-j} k\right) h\left(x-A^{-j} k\right),
$$

with convergence in $\mathcal{S}^{\prime}$. Consequently, if $\varphi, \psi \in \mathcal{S}^{\prime}\left(\mathbb{R}^{n}\right)$ satisfy (2.9), (2.10), then

$$
f=\sum_{Q \in \mathcal{Q}}\left\langle f, \varphi_{Q}\right\rangle \psi_{Q}, \quad \text { for any } f \in \mathcal{S}^{\prime} / \mathcal{P}
$$


where the convergence of the above series, as well as the equality, is in $\mathcal{S}^{\prime} / \mathcal{P}$. More precisely, there exists a sequence of polynomials $\left\{P_{k}\right\}_{k=1}^{\infty} \subset \mathcal{P}$ and $P \in \mathcal{P}$ such that

$$
f=\lim _{k \rightarrow \infty}\left(\sum_{Q \in \mathcal{Q},|\operatorname{det} A|^{-k} \leq|Q| \leq|\operatorname{det} A|^{k}}\left\langle f, \varphi_{Q}\right\rangle \psi_{Q}+P_{k}\right)+P,
$$

with convergence in $\mathcal{S}^{\prime}$.

Proof. The proof of Lemma 2.8 is a straightforward adaptation of [28, Lemma 6.10], which is included for completeness. Since $g \in \mathcal{S}^{\prime}\left(\mathbb{R}^{n}\right)$ has compact support in the Fourier domain, $g$ is regular. More precisely, the distribution $g$ is a slowly increasing (at most polynomially fast) and infinitely differentiable function. Hence, $g\left(A^{-j} k\right)$ is well defined for each $k \in \mathbb{Z}$, and the integral defining $(g * h)(x)$ converges absolutely since $h \in \mathcal{S}\left(\mathbb{R}^{n}\right)$.

First, we suppose that $g \in \mathcal{S}\left(\mathbb{R}^{n}\right)$ and expand $\hat{g}$ in the Fourier orthonormal basis

$$
\left\{\frac{|\operatorname{det} A|^{-j / 2}}{(2 \pi)^{n / 2}} e^{-i\left\langle A^{-j} k, \xi\right\rangle}\right\}_{k \in \mathbb{Z}^{n}}
$$

of $L^{2}\left(\left(A^{*}\right)^{j}[-\pi, \pi]^{n}\right)$,

$$
\hat{g}(\xi)=\sum_{k \in \mathbb{Z}^{n}} \frac{|\operatorname{det} A|^{-j}}{(2 \pi)^{n}}\left(\int_{\left(A^{*}\right)^{j}[-\pi, \pi]^{n}} \hat{g}(y) e^{i\left\langle A^{-j} k, y\right\rangle} d y\right) e^{-i\left\langle A^{-j} k, \xi\right\rangle} .
$$

Since $\hat{g}$ is supported in $\left(A^{*}\right)^{j}[-\pi, \pi]^{n}$, we can replace $\left(A^{*}\right)^{j}[-\pi, \pi]^{n}$ by $\mathbb{R}^{n}$ in the above integral so that we have

$$
\hat{g}(\xi)=\sum_{k \in \mathbb{Z}^{n}}|\operatorname{det} A|^{-j} g\left(A^{-j} k\right) e^{-i\left\langle A^{-j} k, \xi\right\rangle} \quad \text { for } \quad \xi \in\left(A^{*}\right)^{j}[-\pi, \pi]^{n},
$$

by the Fourier inversion formula. Since supp $\hat{h} \subset\left(A^{*}\right)^{j}[-\pi, \pi]^{n}$, we can replace $\hat{g}$ by its periodic extension without altering the product $\hat{g} \hat{h}$. Using $g * h=(\hat{g} \hat{h})$, we obtain

$$
\begin{aligned}
(g * h)(x) & =\sum_{k \in \mathbb{Z}^{n}}|\operatorname{det} A|^{-j} g\left(A^{-j} k\right)\left(e^{-i\left\langle A^{-j} k, \xi\right\rangle} \hat{h}(\xi) \check{)}(x)\right. \\
& =\sum_{k \in \mathbb{Z}^{n}}|\operatorname{det} A|^{-j} g\left(A^{-j} k\right) h\left(x-A^{-j} k\right) .
\end{aligned}
$$

The case of a general $g \in \mathcal{S}^{\prime}$ is obtained from the result just proved by the standard regularization argument. For $\delta>0$, let $g_{\delta}(x)=\gamma(\delta x) g(x)$, where $\gamma \in$ $\mathcal{S}\left(\mathbb{R}^{n}\right)$ satisfies $\gamma(0)=1$, and supp $\hat{\gamma}$ is compact. Since $g_{\delta}(x) \in \mathcal{S}\left(\mathbb{R}^{n}\right)$, we have

$$
\left(g_{\delta} * h\right)(x)=\sum_{k \in \mathbb{Z}^{n}}|\operatorname{det} A|^{-j} g_{\delta}\left(A^{-j} k\right) h\left(x-A^{-j} k\right) .
$$

Using the Lebesgue Dominated Convergence Theorem and taking the limit as $\delta \rightarrow$ 0 , we obtain (2.22). It is clear that the series (2.22) converges pointwise and in $\mathcal{S}^{\prime}$.

Finally, to show (2.23), take any $j \in \mathbb{Z}$. Let $g=f * \tilde{\varphi}_{j}, h=\psi_{j}$, and $\tilde{\varphi}(x)=$ $\overline{\varphi(-x)}$. By (2.22), $\left(f * \tilde{\varphi}_{j}\right)\left(A^{-j} k\right)=|\operatorname{det} A|^{j / 2}\left\langle f, \varphi_{Q_{j, k}}\right\rangle$, and $\psi_{j}\left(x-A^{-j} k\right)=$ $|\operatorname{det} A|^{j / 2} \psi_{Q_{j, k}}$, we have

$$
f * \tilde{\varphi}_{j} * \psi_{j}=\sum_{k \in \mathbb{Z}^{n}}\left\langle f, \varphi_{Q_{j, k}}\right\rangle \psi_{Q_{j, k}} .
$$


Combining Lemma 2.6, (2.9), (2.10), and summing the above over $j \in \mathbb{Z}$, yields (2.23).

\section{TRIEBEL-LIZORKIN SPACES}

In this section we define the weighted anisotropic Triebel-Lizorkin spaces using the Littlewood-Paley decomposition associated with general expansive dilation matrices.

3.1. Homogeneous Triebel-Lizorkin spaces. Motivated by the classical definition of Triebel-Lizorkin spaces by Triebel 42, 43, Frazier, Jawerth and Weiss 27, 28, and their weighted counterparts by Bui [8, 10], we define anisotropic Triebel-Lizorkin spaces as follows.

Definition 3.1. For $\alpha \in \mathbb{R}, 0<p<\infty, 0<q \leq \infty$, and $w \in A_{\infty}$, we define the weighted anisotropic Triebel-Lizorkin space $\dot{\mathbf{F}}_{p}^{\alpha, q}=\dot{\mathbf{F}}_{p}^{\alpha, q}\left(\mathbb{R}^{n}, A, w d x\right)$ as the collection of all $f \in \mathcal{S}^{\prime} / \mathcal{P}$ such that

$$
\|f\|_{\dot{\mathbf{F}}_{p}^{\alpha, q}}=\left\|\left(\sum_{j \in \mathbb{Z}}\left(|\operatorname{det} A|^{j \alpha}\left|f * \varphi_{j}\right|\right)^{q}\right)^{1 / q}\right\|_{L^{p}(w d x)}<\infty,
$$

where $\varphi \in \mathcal{S}\left(\mathbb{R}^{n}\right)$ satisfies (3.2) and (3.3),

$$
\begin{aligned}
& \operatorname{supp} \hat{\varphi}:=\overline{\left\{\xi \in \mathbb{R}^{n}: \hat{\varphi}(\xi) \neq 0\right\}} \subset[-\pi, \pi]^{n} \backslash\{0\}, \\
& \sup _{j \in \mathbb{Z}}\left|\hat{\varphi}\left(\left(A^{*}\right)^{j} \xi\right)\right|>0 \quad \text { for all } \xi \in \mathbb{R}^{n} \backslash\{0\} .
\end{aligned}
$$

To emphasize the dependence on $\varphi$ we will use the notation $\dot{\mathbf{F}}_{p}^{\alpha, q}\left(\mathbb{R}^{n}, A, w d x\right)(\varphi)$ for (3.1). Later we will show that this definition is independent of $\varphi$.

The sequence space $\dot{\mathbf{f}}_{p}^{\alpha, q}=\dot{\mathbf{f}}_{p}^{\alpha, q}(A, w d x)$ is the collection of all complex-valued sequences $s=\left\{s_{Q}\right\}_{Q \in \mathcal{Q}}$ such that

$$
\|s\|_{\dot{\mathbf{f}}_{p}^{\alpha, q}}=\left\|\left(\sum_{Q \in \mathcal{Q}}\left(|Q|^{-\alpha}\left|s_{Q}\right| \tilde{\chi}_{Q}\right)^{q}\right)^{1 / q}\right\|_{L^{p}(w d x)}<\infty,
$$

where $\tilde{\chi}_{Q}=|Q|^{-1 / 2} \chi_{Q}$ is the $L^{2}$-normalized characteristic function of the dilated cube $Q$.

For the basic properties of $\mathcal{S}^{\prime} / \mathcal{P}$, we refer to [42, Section 5.1]. Here, we only recall that $\mathcal{S}^{\prime} / \mathcal{P}$ can be identified with the space of all continuous functionals on the closed subspace $\mathcal{S}_{0}\left(\mathbb{R}^{n}\right)$ of the Schwartz class $\mathcal{S}\left(\mathbb{R}^{n}\right)$ given by

$$
\mathcal{S}_{0}\left(\mathbb{R}^{n}\right)=\left\{\phi \in \mathcal{S}: \int \phi(x) x^{\alpha} d x=0 \text { for all multi-indices } \alpha\right\} .
$$

Equivalently, $\mathcal{S}_{0}\left(\mathbb{R}^{n}\right)$ is defined as a collection of $\phi \in \mathcal{S}$ such that semi-norms

$$
\|\phi\|_{M}=\sup _{|\beta| \leq M} \sup _{\xi \in \mathbb{R}^{n}}\left|\partial^{\beta} \hat{\phi}(\xi)\right|\left(|\xi|^{M}+|\xi|^{-M}\right)<\infty \quad \text { for any } M \in \mathbb{N} .
$$

Moreover, semi-norms $\|\cdot\|_{M}$ generate a topology of a locally convex space on $\mathcal{S}_{0}\left(\mathbb{R}^{n}\right)$.

We will make an extensive use of the following technical lemma. The proof of Lemma 3.1 can be found in Section 6 
Lemma 3.1. Suppose $K$ is a compact subset of $\mathbb{R}^{n}, 0<p<\infty$, and $w \in A_{\infty}$. Then there exist $C, N>0$ such that

$$
\sup _{x \in \mathbb{R}^{n}} \frac{|f(x)|}{(1+|x|)^{N}} \leq C|| f \|_{L^{p}(w)} \quad \text { for all } f \in \mathcal{S}^{\prime} \text { with } \operatorname{supp} \hat{f} \subset K .
$$

As one of the consequences of Lemma 3.1 we can conclude the completeness of $\dot{\mathbf{F}}_{p}^{\alpha, q}\left(\mathbb{R}^{n}, A, w d x\right)$ spaces.

Proposition 3.2. The inclusion map $\dot{\mathbf{F}}_{p}^{\alpha, q}=\dot{\mathbf{F}}_{p}^{\alpha, q}\left(\mathbb{R}^{n}, A, w d x\right) \hookrightarrow \mathcal{S}^{\prime} / \mathcal{P}$ is continuous. Moreover, $\dot{\mathbf{F}}_{p}^{\alpha, q}$ equipped with $\|\cdot\|_{\dot{\mathbf{F}}_{p}^{\alpha, q}}$ is a quasi-Banach space, i.e., $\dot{\mathbf{F}}_{p}^{\alpha, q}$ is a complete quasi-normed space.

Proof. The continuity of the inclusion map is shown most easily for $\varphi$ satisfying (2.13) in addition to (3.2). It is a consequence of Lemma 3.1 applied for compact sets $K_{j}=\left(A^{*}\right)^{j} \operatorname{supp} \hat{\varphi}, j \in \mathbb{Z}$, together with the observation that the constants $C=C\left(K_{j}\right)$ in (3.5) are uniformly bounded for $j<0$ and they grow at most as $O\left(|\operatorname{det} A|^{j / s_{1}}\right)$ as $j \rightarrow \infty$ for some $s_{1}>0$. Therefore, there exists $N>0$ such that for any $\phi \in \mathcal{S}$,

$$
\begin{aligned}
\left|\left\langle f * \varphi_{j}, \phi\right\rangle\right| & \leq C\left(K_{j}\right)\left\|f * \varphi_{j}\right\|_{L^{p}(w)}\left\|(1+|x|)^{N} \phi(x)\right\|_{\infty} \\
& \leq C\left(K_{j}\right)|\operatorname{det} A|^{-j \alpha}|| f\left\|_{\dot{\mathbf{F}}_{p}^{\alpha, q}}||(1+|x|)^{N} \phi(x)\right\|_{\infty} \\
& \leq C|\operatorname{det} A|^{|j|\left(1 / s_{1}+|\alpha|\right)}\|f\|_{\dot{\mathbf{F}}_{p}^{\alpha, q}} \sup _{|\beta| \leq n+1,|\gamma| \leq N}\|\hat{\phi}\|_{\beta, \gamma} .
\end{aligned}
$$

Using techniques similar to the proof of Lemma 2.6, it is then not very difficult to show that there exists $M>0$ and $s_{2}>0$ such that

$$
\left|\left\langle f * \varphi_{j}, \phi\right\rangle\right| \leq\left. C|| f\right|_{\dot{\mathbf{F}}_{p}^{\alpha, q}}|\operatorname{det} A|^{-|j| s_{2}}|| \phi||_{M},
$$

where $\|\phi\|_{M}$ is given by (3.4). Indeed, the idea behind showing (3.7) is as follows. Choose $h \in \mathcal{S}\left(\mathbb{R}^{n}\right)$ such that $\hat{h}(\xi)=1$ for all $\xi \in \operatorname{supp} \hat{\varphi}$ and $\operatorname{supp} \hat{h} \subset\{\xi: 1 / r<$ $\mid \xi<r\}$ for some $r>0$. Then using $\left\langle f * \varphi_{j}, \phi\right\rangle=\left\langle f * \varphi_{j}, h_{j} * \phi\right\rangle$ one can replace the semi-norms $\|\hat{\phi}\|_{\beta, \gamma}$, where $|\beta| \leq n+1$ and $|\gamma| \leq N$, by $\left.\left\|\hat{h}\left(\left(A^{*}\right)^{-j} \cdot\right) \hat{\phi}(\cdot)\right\|\right|_{\beta, \gamma}$ in (3.6). Then techniques from the proof of Lemma 2.6 can be used to show (3.7). For more details about deriving (3.7), we refer the reader to [7, Proposition 3.3].

Thus, (3.7) and Lemma 2.6 yield

$$
|\langle f, \phi\rangle| \leq \sum_{j \in \mathbb{Z}}\left|\left\langle f * \varphi_{j}, \phi\right\rangle\right| \leq C \sum_{j \in \mathbb{Z}}\|f\|_{\dot{\mathbf{F}}_{p}^{\alpha, q}}|\operatorname{det} A|^{-|j| s_{2}}\|\phi\|_{M} \leq\left. C|| f\right|_{\dot{\mathbf{F}}_{p}^{\alpha, q}}\|\phi\|_{M},
$$

which shows that $i: \dot{\mathbf{F}}_{p}^{\alpha, q} \hookrightarrow \mathcal{S}^{\prime} / \mathcal{P}$ is continuous. Once the continuity of the inclusion map $i$ is established, the completeness of $\dot{\mathbf{F}}_{p}^{\alpha, q}\left(\mathbb{R}^{n}, A, w d x\right)$ is immediate by Fatou's Lemma and Lemma 2.6 .

In the case when $\varphi$ satisfies only (3.2) and (3.3), one must use a variant of Lemma 2.6. where (2.14) is replaced by

$$
f=\sum_{j \in \mathbb{Z}} f * \varphi_{j} * \tilde{\psi}_{j}, \quad \text { convergence in } \mathcal{S}^{\prime} / \mathcal{P},
$$

where $\psi$ is as in Lemma 3.6 and $\tilde{\psi}(x)=\overline{\psi(-x)}$. This completes the proof of Proposition 3.2 . 
3.2. Wavelet transforms for $\dot{\mathbf{F}}_{p}^{\alpha, q}\left(\mathbb{R}^{n}, A, w d x\right)$. Suppose that $\varphi, \psi \in \mathcal{S}\left(\mathbb{R}^{n}\right)$ are such that $\operatorname{supp} \hat{\varphi}, \operatorname{supp} \hat{\psi}$ are compact and bounded away from the origin.

Definition 3.2. The $\varphi$-transform $S_{\varphi}$, often called the analysis transform, is the map taking each $f \in \mathcal{S}^{\prime}\left(\mathbb{R}^{n}\right) / \mathcal{P}$ to the sequence $S_{\varphi} f=\left\{\left(S_{\varphi} f\right)_{Q}\right\}_{Q \in \mathcal{Q}}$ defined by $\left(S_{\varphi} f\right)_{Q}=\left\langle f, \varphi_{Q}\right\rangle$. This is well defined, since $\int x^{\gamma} \varphi_{Q}(x) d x=0$ for any multi-index $\gamma$. Here, we follow the pairing convention which is consistent with the usual scalar product in $L^{2}\left(\mathbb{R}^{n}\right)$, i.e., $\langle f, \varphi\rangle=f(\bar{\varphi})$ for $f \in \mathcal{S}^{\prime}$ and $\varphi \in \mathcal{S}$. The inverse $\varphi$ transform, $T_{\psi}$, often called the synthesis transform, is the map taking the sequence $s=\left\{s_{Q}\right\}_{Q \in \mathcal{Q}}$ to $T_{\psi} s=\sum_{Q \in \mathcal{Q}} s_{Q} \psi_{Q}$. We will show later that $T_{\psi} s$ is well defined for $s \in \dot{\mathbf{f}}_{p}^{\alpha, q}$.

Given a sequence $s=\left\{s_{Q}\right\}_{Q}, 0<r<\infty$, and $\lambda>0$, define the sequence $s_{r, \lambda}^{*}=\left\{\left(s_{r, \lambda}^{*}\right)_{Q}\right\}_{Q}$ by

$$
\left(s_{r, \lambda}^{*}\right)_{Q}=\left(\sum_{P \in \mathcal{Q},|P|=|Q|}\left|s_{P}\right|^{r} /\left(1+|Q|^{-1} \rho_{A}\left(x_{Q}-x_{P}\right)\right)^{\lambda}\right)^{1 / r} .
$$

Clearly, we always have $\left|s_{Q}\right| \leq\left(s_{r, \lambda}^{*}\right)_{Q}$ for any $Q \in \mathcal{Q}$.

In order to prove the boundedness of $S_{\varphi}$ and $T_{\psi}$, we need the following two lemmas.

Lemma 3.3. Suppose $\alpha \in \mathbb{R}, 0<p<\infty, 0<q \leq \infty$, and $w \in A_{p_{0}}$. Then for any $r>0$ and $\lambda>\max \left(1, r / q, r p_{0} / p\right)$, there is a constant $C>0$ such that

$$
\|s\|_{\dot{\mathbf{f}}_{p}^{\alpha, q}(A, w d x)} \leq\left\|s_{r, \lambda}^{*}\right\|_{\dot{\mathbf{f}}_{p}^{\alpha, q}(A, w d x)} \leq C\|s\|_{\dot{\mathbf{f}}_{p}^{\alpha, q}(A, w d x)} \quad \text { for all } s=\left\{s_{Q}\right\}_{Q} .
$$

Lemma 3.4 (Anisotropic Peetre's inequality). Let $K$ be a compact subset of $\mathbb{R}^{n}$ and $r>0$. Then there exist constants $C_{1}, C_{2}>0$ such that for any $g \in \mathcal{S}^{\prime}\left(\mathbb{R}^{n}\right)$ with supp $\hat{g} \subset K$, we have

$$
\begin{aligned}
\sup _{z \in \mathbb{R}^{n}} \frac{|\nabla g(x-z)|}{\left(1+\rho_{A}(z)\right)^{1 / r}} & \leq C_{1} \sup _{z \in \mathbb{R}^{n}} \frac{|g(x-z)|}{\left(1+\rho_{A}(z)\right)^{1 / r}} \\
& \leq C_{2}\left[\left(M_{\rho_{A}}|g|^{r}\right)(x)\right]^{1 / r} \quad \text { for all } x \in \mathbb{R}^{n} .
\end{aligned}
$$

The proofs of the above results can be found in Section 6. The next result is a generalization of the fundamental result of Frazier and Jawerth saying that the following diagram is commutative for $\varphi$ and $\psi$ satisfying (2.9) and (2.10):

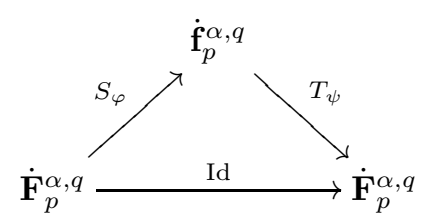

Theorem 3.5. Suppose $\alpha \in \mathbb{R}, 0<p<\infty, 0<q \leq \infty, w \in A_{\infty}$, and $\varphi, \psi \in \mathcal{S}\left(\mathbb{R}^{n}\right)$ are such that $\operatorname{supp} \hat{\varphi}$, supp $\hat{\psi}$ are compact and bounded away from the origin. Then the operators $S_{\varphi}: \dot{\mathbf{F}}_{p}^{\alpha, q}\left(\mathbb{R}^{n}, A, w d x\right)(\tilde{\varphi}) \rightarrow \dot{\mathbf{f}}_{p}^{\alpha, q}(A, w d x)$ and $T_{\psi}: \dot{\mathbf{f}}_{p}^{\alpha, q}(A, w d x) \rightarrow \dot{\mathbf{F}}_{p}^{\alpha, q}\left(\mathbb{R}^{n}, A, w d x\right)(\varphi)$ are bounded, $\tilde{\varphi}(x)=\overline{\varphi(-x)}$. In addition, if $\varphi, \psi$ satisfy (2.9), (2.10) then $T_{\psi} \circ S_{\varphi}$ is the identity on $\dot{\mathbf{F}}_{p}^{\alpha, q}\left(\mathbb{R}^{n}, A, w d x\right)(\varphi)=$ $\dot{\mathbf{F}}_{p}^{\alpha, q}\left(\mathbb{R}^{n}, A, w d x\right)(\tilde{\varphi})$. 
Proof. We will only prove the case of $q<\infty$ and leave details of the easier case $q=\infty$ to the reader.

To prove the boundedness of $T_{\psi}$, take any $s=\left\{s_{Q}\right\}_{Q} \in \dot{\mathbf{f}}_{p}^{\alpha, q}$. We will show that $f=T_{\psi} s=\sum_{Q} s_{Q} \psi_{Q}$ converges in $\dot{\mathbf{F}}_{p}^{\alpha, q}$ and consequently in $\mathcal{S}^{\prime} / \mathcal{P}$. Assume momentarily that $s=\left\{s_{Q}\right\}$ is finitely supported. Since the supports of $\hat{\varphi}$ and $\hat{\psi}$ are bounded and bounded away from the origin, there is an integer $M$ such that $\operatorname{supp} \widehat{\varphi_{j}} \cap \operatorname{supp} \widehat{\psi_{i}}=\emptyset$ for $|i-j|>M$. Therefore,

$$
\left(\varphi_{j} * f\right)(x)=\sum_{i=j-M}^{i=j+M} \sum_{|P|=|\operatorname{det} A|^{-i}} s_{P}\left(\varphi_{j} * \psi_{P}\right)(x) .
$$

Since the functions $\varphi_{l} * \psi, l=-M,-M+1, \ldots, M$ all belong to $\mathcal{S}\left(\mathbb{R}^{n}\right)$, the functions $\varphi_{j} * \psi_{P}$ are uniformly localized on the dilated cubes $P \in \mathcal{Q},|P|=|\operatorname{det} A|^{-i}$, with $|i-j| \leq M$, by the identity

$$
\varphi_{j} * \psi_{P}=\left(\varphi_{j-i} * \psi\right)_{P} \quad \text { for any } P \in \mathcal{Q},|P|=|\operatorname{det} A|^{-i} .
$$

In particular, for any $\lambda>1$, there is a constant $C=C(\lambda)>0$ such that

$$
\left|\left(\varphi_{j} * \psi_{P}\right)(x)\right| \leq C|P|^{-1 / 2}\left(1+\rho_{A}\left(A^{i}\left(x-x_{P}\right)\right)\right)^{-\lambda}
$$

for $P \in \mathcal{Q},|P|=|\operatorname{det} A|^{-i}$, and $|i-j| \leq M$. Given $x \in \mathbb{R}^{n}$ and $i \in \mathbb{Z}$, let $Q^{i}=Q^{i}(x) \in \mathcal{Q}$ be the unique cube such that $x \in Q^{i}$ and $\left|Q^{i}\right|=|\operatorname{det} A|^{-i}$. Therefore,

$$
\begin{aligned}
\left|\varphi_{j} * f(x)\right| \leq C & \sum_{i=j-M}^{i=j+M}|\operatorname{det} A|^{i / 2}\left(\sum_{|P|=|\operatorname{det} A|^{-i}} \frac{\left|s_{P}\right|}{\left(1+\rho_{A}\left(A^{i}\left(x-x_{P}\right)\right)^{\lambda}\right.}\right) \\
\leq C & \sum_{i=j-M}^{i=j+M}\left|Q^{i}\right|^{-1 / 2}\left(\sum_{|P|=\left|Q^{i}\right|} \frac{\left|s_{P}\right|}{\left(1+\left|Q^{i}\right|^{-1} \rho_{A}\left(x_{Q^{i}}-x_{P}\right)\right)^{\lambda}}\right) \\
& =C \sum_{i=j-M}^{i=j+M}\left(s_{1, \lambda}^{*}\right)_{Q^{i}} \tilde{\chi}_{Q^{i}}(x)=C \sum_{i=j-M}^{i=j+M} \sum_{|Q|=|\operatorname{det} A|^{-i}}\left(s_{1, \lambda}^{*}\right)_{Q} \tilde{\chi}_{Q}(x) .
\end{aligned}
$$

By choosing $\lambda>\max \left(1,1 / q, p_{0} / p\right)$, Lemma 3.3 yields

$$
\begin{aligned}
\left\|T_{\psi} s\right\|_{\dot{\mathbf{F}}_{p}^{\alpha, q}} & =\left\|\sum_{Q} s_{Q} \psi_{Q}\right\|_{\dot{\mathbf{F}}_{p}^{\alpha, q}}=\left\|\left(\sum_{j \in \mathbb{Z}}\left(|\operatorname{det} A|^{j \alpha}\left|f * \varphi_{j}\right|\right)^{q}\right)^{1 / q}\right\|_{L^{p}(w)} \\
\leq C & \left\|\sum_{l=-M}^{M}\left(\sum_{j \in \mathbb{Z}} \sum_{|Q|=|\operatorname{det} A|^{-j+l}}\left(|\operatorname{det} A|^{j \alpha}\left|\left(s_{1, \lambda}^{*}\right)_{Q}\right| \tilde{\chi}_{Q}\right)^{q}\right)^{1 / q}\right\| \|_{L^{p}(w)} \\
= & C\left\|\sum_{l=-M}^{M}|\operatorname{det} A|^{l \alpha}\left(\sum_{Q \in \mathcal{Q}}\left(|Q|^{-\alpha}\left|\left(s_{1, \lambda}^{*}\right)_{Q}\right| \tilde{\chi}_{Q}\right)^{q}\right)^{1 / q}\right\|_{L^{p}(w d x)} \leq C\left\|s_{1, \lambda}^{*}\right\|_{\dot{\mathbf{f}}_{p}^{\alpha, q}} \leq C\|s\|_{\dot{\mathbf{f}}_{p}^{\alpha, q}} .
\end{aligned}
$$

To show that the same estimate holds for arbitrary $s \in \dot{\mathbf{f}}_{p}^{\alpha, q}$, we apply the above argument for some special $\varphi$ additionally satisfying (2.13) and (3.2). Then, by the above estimate and Proposition 3.2. $T_{\psi} s=\sum_{Q} s_{Q} \psi_{Q}$ is a well-defined element of $\mathcal{S}^{\prime} / \mathcal{P}$, since sequences with finite support are dense in $\dot{\mathbf{f}}_{p}^{\alpha, q}$ for $p, q<\infty$. Hence, 
by a limiting argument, the above estimate must also hold for arbitrary $s \in \dot{\mathbf{f}}_{p}^{\alpha, q}$, which shows the boundedness of $T_{\psi}$.

To prove the boundedness of $S_{\varphi}$, suppose that $f \in \dot{\mathbf{F}}_{p}^{\alpha, q}\left(\mathbb{R}^{n}, A, w d x\right)(\tilde{\varphi})$. Then, for any $x \in \mathbb{R}^{n}, j \in \mathbb{Z}$, and $r>0$, we have a pointwise estimate

$$
\begin{array}{r}
\sum_{|Q|=|\operatorname{det} A|^{-j}}|Q|^{-\alpha}\left|\left(S_{\varphi} f\right)_{Q}\right| \tilde{\chi}_{Q}(x)=|\operatorname{det} A|^{j \alpha} \sum_{|Q|=|\operatorname{det} A|^{-j}}\left|\left(\tilde{\varphi}_{j} * f\right)\left(x_{Q}\right)\right| \chi_{Q}(x) \\
\leq|\operatorname{det} A|^{j \alpha} \sum_{|Q|=|\operatorname{det} A|^{-j}} \sup _{y \in Q}\left|\left(\tilde{\varphi}_{j} * f\right)(y)\right| \chi_{Q}(x) \\
\leq C|\operatorname{det} A|^{j \alpha} \sup _{z \in Q-Q} \frac{\left|\left(\tilde{\varphi}_{j} * f\right)(x-z)\right|}{\left(1+\rho_{A}\left(A^{j} z\right)\right)^{1 / r}},
\end{array}
$$

where $\tilde{\varphi}(x)=\overline{\varphi(-x)}$, since $z \in Q-Q \subset B_{\rho_{A}}\left(0,2 H|\operatorname{det} A|^{-j}\right)$. Therefore,

$$
\begin{aligned}
& \left.\sum_{|Q|=\mid \operatorname{det}}|Q|^{-j}\right|^{-\alpha}\left|\left(S_{\varphi} f\right)_{Q}\right| \tilde{\chi}_{Q}(x) \\
& \quad \leq C \sup _{z \in \mathbb{R}^{n}} \frac{\left|\left(\tilde{\varphi}_{j} * f\right)(x-z)\right|}{\left(1+\rho_{A}\left(A^{j} z\right)\right)^{1 / r}}=C \sup _{z \in \mathbb{R}^{n}} \frac{\left|g\left(A^{j} x-z\right)\right|}{\left(1+\rho_{A}(z)\right)^{1 / r}} \\
& \leq C\left(M_{\rho_{A}}|g|^{r}\left(A^{j} x\right)\right)^{1 / r}=C\left(M_{\rho_{A}}\left|\tilde{\varphi}_{j} * f\right|^{r}(x)\right)^{1 / r},
\end{aligned}
$$

where $g(x)=\left(\tilde{\varphi}_{j} * f\right)\left(A^{-j} x\right)$. We used here the dilation invariance of $M_{\rho_{A}}$,

$$
M_{\rho_{A}}(g)\left(A^{j} x\right)=M_{\rho_{A}}\left(g\left(A^{j} \cdot\right)\right)(x), \quad x \in \mathbb{R}^{n}, j \in \mathbb{Z},
$$

and Lemma 3.4 (Peetre's inequality), since supp $\hat{g}$ is compact and it is (independently of $j \in \mathbb{Z}$ ) contained in $\operatorname{supp} \widehat{\tilde{\varphi}}$.

By the Fefferman-Stein vector-valued inequality with $0<r<\min \left(p / p_{0}, q\right)$, we have

$$
\begin{gathered}
\left\|S_{\varphi} f\right\|_{\dot{\mathbf{f}}_{p}^{\alpha, q}(A, w d x)} \leq C\left\|\left(\sum_{j \in \mathbb{Z}}\left(|\operatorname{det} A|^{j \alpha} M_{\rho_{A}}^{1 / r}\left(\left|\tilde{\varphi}_{j} * f\right|^{r}\right)\right)^{q}\right)^{1 / q}\right\|_{L_{p}(w)} \\
=C\left\|\left(\sum_{j \in \mathbb{Z}}\left(M_{\rho_{A}}\left(|\operatorname{det} A|^{j \alpha r}\left|\tilde{\varphi}_{j} * f\right|^{r}\right)\right)^{q / r}\right)^{r / q}\right\|_{L_{p / r}(w)}^{1 / r} \\
\leq C\left\|\left(\sum_{j \in \mathbb{Z}}\left(|\operatorname{det} A|^{j \alpha r}\left|\tilde{\varphi}_{j} * f\right|^{r}\right)^{q / r}\right)^{r / q}\right\|_{L_{p / r}(w)}^{1 / r} \\
=C\left\|\left(\sum_{j \in \mathbb{Z}}\left(|\operatorname{det} A|^{j \alpha}\left|\tilde{\varphi}_{j} * f\right|\right)^{q}\right)^{1 / q}\right\|_{L_{p}(w)}=C\|f\|_{\dot{F}_{p}^{\alpha, q}\left(\mathbb{R}^{n}, A, w d x\right)(\tilde{\varphi})},
\end{gathered}
$$

which shows boundedness of $S_{\varphi}$.

Finally, if we assume additionally that $\varphi$ and $\psi$ satisfy (2.9) and (2.10), then by Lemma 2.8, $T_{\psi} \circ S_{\varphi}$ is the identity on $\dot{\mathbf{F}}_{p}^{\alpha, q}$. More precisely, $\dot{\mathbf{F}}_{p}^{\alpha, q}\left(\mathbb{R}^{n}, A, w d x\right)(\tilde{\varphi}) \hookrightarrow$ $\dot{\mathbf{F}}_{p}^{\alpha, q}\left(\mathbb{R}^{n}, A, w d x\right)(\varphi)$ is a bounded inclusion. Hence, by reversing the roles of $\varphi$ and $\tilde{\varphi}$ we have

$$
\dot{\mathbf{F}}_{p}^{\alpha, q}\left(\mathbb{R}^{n}, A, w d x\right)(\tilde{\varphi})=\dot{\mathbf{F}}_{p}^{\alpha, q}\left(\mathbb{R}^{n}, A, w d x\right)(\varphi),
$$

which completes the proof of Theorem 3.5 . 
Lemma 3.6. Suppose $\varphi \in \mathcal{S}$ satisfies (3.2) and (3.3). Then there exists $\psi \in \mathcal{S}$ such that (2.9) and (2.10) are satisfied.

Proof. Let $h(\xi)=\sum_{j \in \mathbb{Z}}\left|\hat{\varphi}\left(\left(A^{*}\right)^{j} \xi\right)\right|^{2}$. By (3.2) and the fact that $A^{*}$ is expansive, for any $\xi_{0} \neq 0$, there is a neighborhood $U$ of $\xi_{0}$, such that for $\xi \in U$, only a finite number of terms in the sum defining $h(\xi)$ are non-zero. Therefore, $h(\xi)$ is $C^{\infty}$ on $\mathbb{R}^{n} \backslash\{0\}$. Moreover, by a compactness argument and (3.3), there are constants $c_{1}$, $c_{2}$ such that

$$
0<c_{1} \leq h(\xi) \leq c_{2}<\infty \quad \text { for all } \xi \in \mathbb{R}^{n} \backslash\{0\} .
$$

Define $\psi$, by $\hat{\psi}(\xi)=\overline{\hat{\varphi}(\xi)} / h(\xi)$. An instant verification shows that the Calderón condition (2.10) holds and $\psi \in \mathcal{S}$, since $\hat{\psi}$ is $C^{\infty}$ on $\mathbb{R}^{n}$ and $\operatorname{supp} \hat{\psi}=\operatorname{supp} \hat{\varphi} \subset$ $[-\pi, \pi]^{n} \backslash\{0\}$.

Corollary 3.7. Suppose that $\alpha \in \mathbb{R}, 0<p<\infty, 0<q \leq \infty$, and $w \in A_{\infty}$. Then the space $\dot{\mathbf{F}}_{p}^{\alpha, q}$ is well defined in the sense that, for any $\varphi^{1}$ and $\varphi^{2}$ satisfying (3.2) and (3.3), their associated quasi-norms in $\dot{\mathbf{F}}_{p}^{\alpha, q}\left(\mathbb{R}^{n}, A, w d x\right)\left(\varphi^{i}\right), i=1,2$, are equivalent, i.e., there exist constants $C_{1}, C_{2}>0$ such that

$$
C_{1}\|f\|_{\dot{\mathbf{F}}_{p}^{\alpha, q}\left(\mathbb{R}^{n}, A, w d x\right)\left(\varphi^{1}\right)} \leq\|f\|_{\dot{\mathbf{F}}_{p}^{\alpha, q}\left(\mathbb{R}^{n}, A, w d x\right)\left(\varphi^{2}\right)} \leq C_{2}\|f\|_{\dot{\mathbf{F}}_{p}^{\alpha, q}\left(\mathbb{R}^{n}, A, w d x\right)\left(\varphi^{1}\right)} .
$$

Proof. Suppose $\varphi^{1}$ and $\varphi^{2}$ each satisfy (3.2) and (3.3). Then by Lemma 3.6, it is possible to find $\psi^{1}$ and $\psi^{2}$ so that (2.9) and (2.10) are satisfied for each pair $\varphi^{i}$, $\psi^{i}, i=1,2$. Then by Lemma 2.8

$$
\begin{array}{r}
\|f\|_{\dot{\mathbf{F}}_{p}^{\alpha, q}\left(\mathbb{R}^{n}, A, w d x\right)\left(\varphi^{1}\right)}=\left\|\left(T_{\tilde{\psi}^{2}} \circ S_{\tilde{\varphi}^{2}}\right) f\right\|_{\dot{\mathbf{F}}_{p}^{\alpha, q}\left(\mathbb{R}^{n}, A, w d x\right)\left(\varphi^{1}\right)} \leq C\left\|S_{\tilde{\varphi}^{2}} f\right\|_{\dot{\mathbf{f}}_{p}^{\alpha, q}(A, w d x)} \\
\leq C\|f\|_{\dot{\mathbf{F}}_{p}^{\alpha, q}\left(\mathbb{R}^{n}, A, w d x\right)\left(\varphi^{2}\right)},
\end{array}
$$

by the boundedness of $S_{\tilde{\varphi}^{2}}$ and $T_{\tilde{\psi}^{2}}$, since the pair $\tilde{\varphi}^{2}, \tilde{\psi}^{2}$ satisfies (2.9) and (2.10). Reversing the roles of $\varphi^{1}$ and $\varphi^{2}$ yields (3.9).

Remark 3.1. Suppose that $\varphi \in \mathcal{S}$ is such that supp $\hat{\varphi}$ is compact and bounded away from the origin. Hence, $\varphi$ may not necessarily satisfy (3.2) or (3.3) and consequently $\dot{\mathbf{F}}_{p}^{\alpha, q}\left(\mathbb{R}^{n}, A, w d x\right)(\varphi)$ may not be a complete quasi-normed space. Nevertheless, from the proof of Corollary 3.7, it follows that we still have a constant $C>0$ depending on $\varphi$ such that

$$
\|f\|_{\dot{\mathbf{F}}_{p}^{\alpha, q}\left(\mathbb{R}^{n}, A, w d x\right)(\varphi)} \leq C\|f\|_{\dot{\mathbf{F}}_{p}^{\alpha, q}} \quad \text { for all } f \in \dot{\mathbf{F}}_{p}^{\alpha, q} .
$$

We will also need the following very useful fact, which resolves all sorts of issues caused by the fact that the elements of $\dot{\mathbf{F}}_{p}^{\alpha, q}$ are equivalence classes of tempered distributions $\mathcal{S}^{\prime}$ modulo polynomials $\mathcal{P}$. This result guarantees the existence of canonical representatives of elements in $\dot{\mathbf{F}}_{p}^{\alpha, q}$ modulo polynomials of degree $\leq L=$ $\left\lfloor\alpha / \zeta_{-}\right\rfloor$. Proposition 3.8 is a generalization of [27, Remark B.4] and [36, pp. 52-56] in the unweighted case and [10, Proposition 1.1] in the weighted case.

Proposition 3.8. Suppose that $\alpha \in \mathbb{R}, 0<p<\infty, 0<q \leq \infty, w \in A_{\infty}$, and $f \in \dot{\mathbf{F}}_{p}^{\alpha, q}\left(\mathbb{R}^{n}, A, w d x\right)$. For any $\varphi^{1} \in \mathcal{S}\left(\mathbb{R}^{n}\right)$ such that $\operatorname{supp} \widehat{\varphi^{1}}$ is compact and bounded away from the origin, and (2.13) holds, there exists a sequence of polynomials $\left\{P_{k}^{1}\right\}_{k=1}^{\infty}$ with $\operatorname{deg} P_{k}^{1} \leq L=\left\lfloor\alpha / \zeta_{-}\right\rfloor$such that

$$
g^{1}:=\lim _{k \rightarrow \infty}\left(\sum_{j=-k}^{\infty}\left(\varphi^{1}\right)_{j} * f+P_{k}^{1}\right)
$$


exists in $\mathcal{S}^{\prime}$. Moreover, if $g^{2}$ is the corresponding limit in (3.10) for some other $\varphi^{2} \in \mathcal{S}\left(\mathbb{R}^{n}\right)$ such that $\operatorname{supp} \widehat{\varphi^{2}}$ is compact and bounded away from the origin, and (2.13) holds, then

$$
g^{1}-g^{2} \in \mathcal{P} \quad \text { and } \quad \operatorname{deg}\left(g^{1}-g^{2}\right) \leq L .
$$

Proof. Note that Lemma 2.6 already guarantees the existence of polynomials $\left\{P_{k}^{1}\right\}_{k=1}^{\infty}$ with $\operatorname{deg} P_{k}^{1} \leq d$ for some $d \geq 0$ such that (3.10) holds. However, it is not clear why $d$ could be chosen to be $\leq L=\left\lfloor\alpha / \zeta_{-}\right\rfloor$and why (3.11) holds. Nevertheless, by Lemma 2.6 we know that $\sum_{j=0}^{\infty}\left(\varphi^{1}\right)_{j} * f$ converges in $\mathcal{S}^{\prime}$.

Let $N>0$ be the constant guaranteed by Lemma 3.1 for $K=\bigcup_{j<0}\left(A^{*}\right)^{j}\left(\operatorname{supp} \widehat{\varphi^{1}}\right)$. Then, for any $j<0$ and a multi-index $\beta$, by Remark 3.1 and Lemma 3.1 .

$$
\begin{aligned}
\sup _{x \in \mathbb{R}^{n}} & \frac{\left|\partial^{\beta}\left(\left(\varphi^{1}\right)_{j} * f\right)(x)\right|}{(1+|x|)^{N}} \\
& \leq C\left\|\partial^{\beta}\left(\left(\varphi^{1}\right)_{j} * f\right)\right\|_{L^{p}(w)}=C\left\|\left(\partial^{\beta}\left(\varphi^{1}\right)_{j}\right) * f\right\|_{L^{p}(w)} \\
& \leq C \sum_{|\gamma|=|\beta|}\left|a_{\gamma, j}\right|\left\|\left(\partial^{\gamma} \varphi^{1}\right)_{j} * f\right\|_{L^{p}(w)} \\
& \leq C\left(\lambda_{-}\right)^{j|\beta|}|\operatorname{det} A|^{-j \alpha} \sum_{|\gamma|=|\beta|}\|f\|_{\dot{\mathbf{F}}_{p}^{\alpha, \infty}\left(\mathbb{R}^{n}, A, w\right)\left(\partial^{\gamma} \varphi^{1}\right)} \\
& \leq C|\operatorname{det} A|^{j\left(|\beta| \zeta_{-}-\alpha\right)} \sum_{|\gamma|=|\beta|}\|f\|_{\dot{\mathbf{F}}_{p}^{\alpha, q}\left(\mathbb{R}^{n}, A, w d x\right)\left(\partial^{\gamma} \varphi^{1}\right)} \\
& \leq C|\operatorname{det} A|^{j\left(|\beta| \zeta_{-}-\alpha\right)} \|\left. f\right|_{\dot{\mathbf{F}}_{p}^{\alpha, q} .}
\end{aligned}
$$

Here, we used that for any $\varphi \in \mathcal{S}$ and a multi-index $\beta$, there exists a constant $C>0$ such that for all $j \geq 0$, we have

$$
\partial^{\beta} \varphi_{j}(x)=\sum_{|\gamma|=|\beta|} a_{\gamma, j}\left(\partial^{\gamma} \varphi\right)_{j}(x), \quad \text { where }\left|a_{\gamma, j}\right| \leq C\left(\lambda_{-}\right)^{j|\gamma|} .
$$

This follows from the chain rule and the estimate $\| A^{j}|| \gamma \mid \leq C\left(\lambda_{-}\right)^{j|\gamma|}$ for $j \leq 0$; see also 6, the proof of Lemma 5.2].

Therefore, by (3.12),$\sum_{j<0} \partial^{\beta}\left(\left(\varphi^{1}\right)_{j} * f\right)$ converges in $\mathcal{S}^{\prime}$ for any $|\beta|>L$, since $|\beta| \zeta_{-}-\alpha>0$. Consequently, Proposition 2.7 yields polynomials $\left\{P_{k}^{1}\right\}_{k=1}^{\infty}$ with $\operatorname{deg} P_{k}^{1} \leq L$ and $g^{1} \in \mathcal{S}^{\prime}$ such that (3.10) holds.

To show (3.11), let $\varphi^{2}$ be another function satisfying hypotheses of Proposition 3.8 and let $g^{2} \in \mathcal{S}^{\prime}$ be the corresponding limit of (3.10) for some sequence of polynomials $\left\{P_{k}^{2}\right\}_{k=1}^{\infty}$ with $\operatorname{deg} P_{k}^{2} \leq L$. Since $g^{1}$ and $g^{2}$ represent the same equivalence class in $\mathcal{S}^{\prime} / \mathcal{P}$ of $f \in \dot{\mathbf{F}}_{p}^{\alpha, q}, g^{1}-g^{2}$ is a polynomial. Let $K=\bigcup_{j<0}\left(A^{*}\right)^{j}\left(\operatorname{supp} \widehat{\varphi^{1}} \cup \operatorname{supp} \widehat{\varphi^{2}}\right)$. Then, by a simple support argument and (2.13),

$$
\operatorname{supp}\left(\sum_{j=-k}^{\infty}\left(\left(\varphi^{1}\right)_{j} * f-\left(\varphi^{2}\right)_{j} * f\right)\right)^{\wedge} \subset\left(A^{*}\right)^{-k} K \quad \text { for any } k \in \mathbb{Z} .
$$

Let $\varphi \in \mathcal{S}$ be given by

$$
\hat{\varphi}(\xi)=\sum_{j=0}^{\infty} \widehat{\varphi^{1}}\left(\left(A^{*}\right)^{-j} \xi\right)-\widehat{\varphi^{2}}\left(\left(A^{*}\right)^{-j} \xi\right) .
$$


To check that $\varphi \in \mathcal{S}$, note that for every $\xi \in \mathbb{R}^{n}$, only a finite number of terms in (3.15) are non-zero and hence $\hat{\varphi}$ is $C^{\infty}$. The support of $\hat{\varphi}$ is bounded and bounded away from the origin, since $\hat{\varphi}(\xi)=0$ for all $\xi \notin K$, by (2.13). Equivalently, $\varphi$ can be defined as

$$
\varphi(x)=\sum_{j=0}^{\infty}\left(\varphi^{1}\right)_{j}(x)-\left(\varphi^{2}\right)_{j}(x) \quad \text { for } x \neq 0,
$$

where the series converges pointwise for all $x \in \mathbb{R}^{n} \backslash\{0\}$. However, the above series does not converge for $x=0$ (unless $\varphi^{1}(0)=\varphi^{2}(0)$ ) and (3.15) is needed to show that $\varphi \in \mathcal{S}$.

We claim that

$$
\sum_{j=-k}^{\infty}\left(\left(\varphi^{1}\right)_{j} * f-\left(\varphi^{2}\right)_{j} * f\right)=\varphi_{-k} * f
$$

where the series converges in $\mathcal{S}^{\prime}$. Indeed, by (3.15)

$$
\left(\sum_{j=-k}^{\infty}\left(\varphi^{1}\right)_{j}-\left(\varphi^{2}\right)_{j}\right) \hat{(\xi)}=\sum_{j=-k}^{\infty} \widehat{\varphi^{1}}\left(\left(A^{*}\right)^{-j} \xi\right)-\widehat{\varphi^{2}}\left(\left(A^{*}\right)^{-j} \xi\right)=\hat{\varphi}\left(\left(A^{*}\right)^{k} \xi\right)=\widehat{\varphi-k}(\xi) .
$$

Hence, by (3.12) and (3.16), for any $\phi \in \mathcal{S}$ and $|\beta|>L$,

$$
\begin{aligned}
& \left|\left\langle\partial^{\beta}\left(g^{1}-g^{2}\right), \phi\right\rangle\right|=\left|\lim _{k \rightarrow \infty}\left\langle\sum_{j=-k}^{\infty} \partial^{\beta}\left(\left(\varphi^{1}\right)_{j} * f+P_{k}^{1}-\left(\varphi^{2}\right)_{j} * f-P_{k}^{2}\right), \phi\right\rangle\right| \\
& =\lim _{k \rightarrow \infty}\left|\left\langle\sum_{j=-k}^{\infty} \partial^{\beta}\left(\left(\varphi^{1}\right)_{j} * f-\left(\varphi^{2}\right)_{j} * f\right), \phi\right\rangle\right|=\lim _{k \rightarrow \infty}\left|\left\langle\partial^{\beta}\left(\varphi_{-k} * f\right), \phi\right\rangle\right| \\
& \leq \lim _{k \rightarrow \infty} \sup _{x \in \mathbb{R}^{n}} \frac{\left|\partial^{\beta}\left(\varphi_{-k} * f\right)(x)\right|}{(1+|x|)^{N}} \int_{\mathbb{R}^{n}}(1+|x|)^{N}|\phi(x)| d x \\
& \leq\left. C \lim _{k \rightarrow \infty}|\operatorname{det} A|^{-k\left(|\beta| \zeta_{-}-\alpha\right)}|| f\right|_{\dot{\mathbf{F}}_{p}^{\alpha, q}}=0 .
\end{aligned}
$$

This shows (3.11) and completes the proof of Proposition 3.8

As an immediate corollary of Lemma 2.8 and Proposition 3.8, we have

Corollary 3.9. Suppose that $\alpha \in \mathbb{R}, 0<p<\infty, 0<q \leq \infty, w \in A_{\infty}$, and $f \in \dot{\mathbf{F}}_{p}^{\alpha, q}\left(\mathbb{R}^{n}, A, w d x\right)$. Given $\varphi^{1}, \psi^{1} \in \mathcal{S}$ satisfying (2.9) and (2.10), there exists a sequence of polynomials $\left\{P_{k}^{1}\right\}_{k=1}^{\infty}$ with $\operatorname{deg} P_{k}^{1} \leq L=\left\lfloor\alpha / \zeta_{-}\right\rfloor$such that

$$
g^{1}:=\lim _{k \rightarrow \infty}\left(\sum_{Q \in \mathcal{Q},|\operatorname{det} A|^{-k} \leq|Q| \leq|\operatorname{det} A|^{k}}\left\langle f,\left(\varphi^{1}\right)_{Q}\right\rangle\left(\psi^{1}\right)_{Q}+P_{k}^{1}\right)
$$

exists in $\mathcal{S}^{\prime}$. Moreover, if $g^{2}$ is the corresponding limit in (3.17) for some other $\varphi^{2}, \psi^{2} \in \mathcal{S}$ satisfying (2.9) and (2.10), then (3.11) holds.

3.3. Inhomogeneous Triebel-Lizorkin spaces. In this subsection we define inhomogeneous counterparts $\mathbf{F}_{p}^{\alpha, q}$ of homogeneous Triebel-Lizorkin spaces $\dot{\mathbf{F}}_{p}^{\alpha, q}$ and we briefly describe some of their basic properties. In general, it is much easier to prove results for inhomogeneous than homogeneous spaces, since all technical issues involving convergence in $\mathcal{S}^{\prime} / \mathcal{P}$, which preoccupied much of our attention, are nonexistent in the inhomogeneous case. Moreover, all results for inhomogeneous spaces considered here are generally straightforward modifications of the corresponding homogeneous results and therefore we will only outline required changes. 
Definition 3.3. For $\alpha \in \mathbb{R}, 0<p<\infty, 0<q \leq \infty$, and $w \in A_{\infty}$, we define the weighted inhomogeneous anisotropic Triebel-Lizorkin space $\mathbf{F}_{p}^{\alpha, q}=\mathbf{F}_{p}^{\alpha, q}\left(\mathbb{R}^{n}, A\right.$, wdx $)$ as the collection of all $f \in \mathcal{S}^{\prime}$ such that

$$
\|f\|_{\mathbf{F}_{p}^{\alpha, q}}=\|f * \Phi\|_{L^{p}(w)}+\left\|\left(\sum_{j=1}^{\infty}\left(|\operatorname{det} A|^{j \alpha}\left|f * \varphi_{j}\right|\right)^{q}\right)^{1 / q}\right\|_{L^{p}(w)}<\infty,
$$

where $\Phi \in \mathcal{S}\left(\mathbb{R}^{n}\right)$ and $\varphi \in \mathcal{S}\left(\mathbb{R}^{n}\right)$ satisfy (3.2), (3.18), and (3.19),

$$
\begin{aligned}
& \operatorname{supp} \hat{\Phi} \subset[-\pi, \pi]^{n}, \\
& \sup _{j \geq 1}\left\{\left|\hat{\varphi}\left(\left(A^{*}\right)^{-j} \xi\right)\right|,|\hat{\Phi}(\xi)|\right\}>0 \quad \text { for all } \xi \in \mathbb{R}^{n} .
\end{aligned}
$$

As in the homogeneous case, we will see that this definition is independent of $\Phi$ and $\varphi$ as above.

Let $\mathcal{Q}_{0}=\{Q \in \mathcal{Q}:|Q| \leq 1\}$. The sequence space, $\mathbf{f}_{p}^{\alpha, q}=\mathbf{f}_{p}^{\alpha, q}(A, w d x)$, is the collection of all complex-valued sequences $s=\left\{s_{Q}\right\}_{Q \in \mathcal{Q}_{0}}$ such that

$$
\|s\|_{\mathbf{f}_{p}^{\alpha, q}}=\left\|\left(\sum_{|Q| \leq 1}\left(|Q|^{-\alpha}\left|s_{Q}\right| \tilde{\chi}_{Q}\right)^{q}\right)^{1 / q}\right\|_{L^{p}(w)}<\infty,
$$

where $\tilde{\chi}_{Q}=|Q|^{-1 / 2} \chi_{Q}$ is the $L^{2}$-normalized characteristic function of the dilated cube $Q$.

Since $\mathbf{f}_{p}^{\alpha, q}$ is trivially isometrically imbedded in $\dot{\mathbf{f}}_{p}^{\alpha, q}$, virtually all results for $\dot{\mathbf{f}}_{p}^{\alpha, q}$ have immediate analogues for $\mathbf{f}_{p}^{\alpha, q}$. In particular, it is immediate that Lemma 3.3 holds for $\mathbf{f}_{p}^{\alpha, q}$.

We can also define $\varphi$-transform $S_{\varphi}$ and the inverse $\varphi$-transform $T_{\psi}$ corresponding to the inhomogeneous setting.

Definition 3.4. Suppose that $\Phi, \Psi \in \mathcal{S}\left(\mathbb{R}^{n}\right), \varphi, \psi \in \mathcal{S}\left(\mathbb{R}^{n}\right)$ satisfy (3.2), (3.3), and (3.19). Define the inhomogeneous $\varphi$-transform $S_{\varphi}=S_{\Phi, \varphi}$ to be the map taking each $f \in \mathcal{S}^{\prime}\left(\mathbb{R}^{n}\right)$ to the sequence $S_{\varphi} f=\left\{\left(S_{\varphi} f\right)_{Q}\right\}_{Q \in \mathcal{Q}_{0}}$ defined by

$$
\left(S_{\varphi} f\right)_{Q}=\left\langle f, \Phi_{Q}\right\rangle \quad \text { if } \quad|Q|=1, \quad\left(S_{\varphi} f\right)_{Q}=\left\langle f, \varphi_{Q}\right\rangle \quad \text { if } \quad|Q|<1 .
$$

The inhomogeneous inverse $\varphi$-transform $T_{\psi}=T_{\Psi, \psi}$ is the map taking the sequence $s=\left\{s_{Q}\right\}_{Q \in \mathcal{Q}_{0}}$ to $T_{\psi} s=\sum_{|Q|=1} s_{Q} \Psi_{Q}+\sum_{|Q|<1} s_{Q} \psi_{Q}$.

Given a pair $\Phi, \varphi \in \mathcal{S}$ satisfying (3.2), (3.18), and (3.19) one can show that there exists another pair $\Psi, \psi \in \mathcal{S}$ satisfying the same properties such that

$$
\overline{\hat{\Phi}(\xi)} \hat{\Psi}(\xi)+\sum_{j=1}^{\infty} \overline{\hat{\varphi}\left(\left(A^{*}\right)^{-j} \xi\right)} \hat{\psi}\left(\left(A^{*}\right)^{-j} \xi\right)=1 \quad \text { for all } \xi \in \mathbb{R}^{n} .
$$

Indeed, to show (3.20), notice that (3.18) and (3.19) imply that $\varphi$ satisfies (3.3). By Lemma 3.6. there exists $\psi \in \mathcal{S}$ such that (2.9) and (2.10) hold and $\overline{\hat{\varphi}(\xi)} \hat{\psi}(\xi) \geq 0$ for all $\xi \in \mathbb{R}^{n}$. Hence, by a compactness argument and (3.19), it is easy to find a required $\Psi \in \mathcal{S}$ such that (3.20) holds. Moreover, as in Lemma 2.8, we have the representation formula

$$
f=\sum_{|Q|=1}\left\langle f, \Phi_{Q}\right\rangle \Psi_{Q}+\sum_{|Q|<1}\left\langle f, \varphi_{Q}\right\rangle \psi_{Q},
$$

for any $f \in \mathcal{S}^{\prime}\left(\mathbb{R}^{n}\right)$ with convergence in $\mathcal{S}^{\prime}$. 
Finally, one can show that Theorem 3.5 also holds in the inhomogeneous setting. That is, $S_{\varphi}$ is a bounded operator from $\mathbf{F}_{p}^{\alpha, q}$ to $\mathbf{f}_{p}^{\alpha, q}$ and $T_{\psi}$ is a bounded operator from $\mathbf{f}_{p}^{\alpha, q}$ to $\mathbf{F}_{p}^{\alpha, q}$. Moreover, if $\Phi, \Psi \in \mathcal{S}\left(\mathbb{R}^{n}\right)$ and $\varphi, \psi \in \mathcal{S}\left(\mathbb{R}^{n}\right)$ satisfy (3.2), (3.18), and (3.19), then $T_{\psi} \circ S_{\varphi}$ is the identity operator on $\mathbf{F}_{p}^{\alpha, q}$. Indeed, it is not hard to verify that the proof of the boundedness of $S_{\varphi}$ and $T_{\psi}$ is a direct adaption of the proof of Theorem 3.5. It is then clear that the analogue of Corollary 3.7 also holds for $\mathbf{F}_{p}^{\alpha, q}$.

\section{Almost diagonal operators}

In this section we study the class of almost diagonal operators on $\dot{\mathbf{f}}_{p}^{\alpha, q}(A, w d x)$, which was introduced in the dyadic case by Frazier and Jawerth [27. The interest of these operators on $\dot{\mathbf{f}}_{p}^{\alpha, q}$ arises from their close connection to operators on function spaces.

For a quasi-Banach space $X$, let $\mathcal{L}(X)$ be the algebra of bounded linear operators on $X$ with the operator norm. Define the bounded operators $S_{\varphi}^{*}: \mathcal{L}\left(\dot{\mathbf{F}}_{p}^{\alpha, q}\right) \rightarrow$ $\mathcal{L}\left(\dot{\mathbf{f}}_{p}^{\alpha, q}\right)$ and $T_{\psi}^{*}: \mathcal{L}\left(\dot{\mathbf{f}}_{p}^{\alpha, q}\right) \rightarrow \mathcal{L}\left(\dot{\mathbf{F}}_{p}^{\alpha, q}\right)$ by

$$
\begin{array}{ll}
S_{\varphi}^{*}(T)=S_{\varphi} \circ T \circ T_{\psi} & \text { for } T \in \mathcal{L}\left(\dot{\mathbf{F}}_{p}^{\alpha, q}\right), \\
T_{\psi}^{*}(\mathcal{A})=T_{\psi} \circ \mathcal{A} \circ S_{\varphi} & \text { for } \mathcal{A} \in \mathcal{L}\left(\dot{\mathbf{f}}_{p}^{\alpha, q}\right) .
\end{array}
$$

Repeating verbatim the arguments in [27. Section 3] and using Theorem [3.5] we have the following commutative diagram:

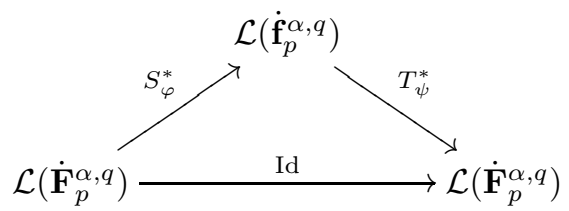

Moreover, if $q \neq \infty$, then any $\mathcal{A} \in \mathcal{L}\left(\dot{\mathbf{f}}_{p}^{\alpha, q}\right)$ is represented by a matrix $\left\{a_{Q P}\right\}_{Q, P \in \mathcal{Q}}$, where $a_{Q P}=\left(\mathcal{A} e^{P}\right)_{Q}$. Here, $e^{P}, P \in \mathcal{Q}$ denotes the standard unit vector in $\dot{\mathbf{f}}_{p}^{\alpha, q}$ defined by $\left(e^{P}\right)_{Q}=\delta_{P Q}$. If $q=\infty$, then despite the fact that not every $\mathcal{A} \in \mathcal{L}\left(\dot{\mathbf{f}}_{p}^{\alpha, q}\right)$ can be represented by a matrix, we claim that for any $B \in \mathcal{L}\left(\dot{\mathbf{F}}_{p}^{\alpha, q}\right)$, an operator $S_{\varphi}^{*} B \in \mathcal{L}\left(\dot{\mathbf{f}}_{p}^{\alpha, q}\right)$ has a matrix representation. Indeed, for any $s \in \dot{\mathbf{f}}_{p}^{\alpha, q}$,

$$
\left(\left(S_{\varphi}^{*}\right)(s)\right)_{Q}=\left(S_{\varphi} B T_{\psi} s\right)_{Q}=\left\langle B \sum_{P} s_{P} \psi_{P}, \varphi_{Q}\right\rangle=\sum_{P} s_{P}\left\langle B \psi_{P}, \varphi_{Q}\right\rangle,
$$

by Proposition 3.2 and Theorem 3.5 . We are now ready to introduce the class of almost diagonal operators on $\dot{\mathbf{f}}_{p}^{\alpha, q}$.

Definition 4.1. Suppose $\alpha \in \mathbb{R}, 0<p<\infty, 0<q \leq \infty, w \in A_{\infty}$, and $r_{0}=$ $\inf \left\{r: w \in A_{r}\right\}$. Let $J=\max \left(1, r_{0} / p, 1 / q\right)$. We say that an operator $\mathcal{A}$, with an associated matrix $\left\{a_{Q P}\right\}_{Q, P \in \mathcal{Q}}$, where $a_{Q P}=\left(\mathcal{A} e^{P}\right)_{Q}$, is an almost diagonal operator on $\dot{\mathbf{f}}_{p}^{\alpha, q}(A, w d x)$, if there exists an $\epsilon>0$ such that

$$
\sup _{Q, P \in \mathcal{Q}}\left|a_{Q P}\right| / \kappa_{Q P}(\epsilon)<\infty,
$$


where

$$
\kappa_{Q P}(\epsilon)=\left(\frac{|Q|}{|P|}\right)^{\alpha}\left(1+\frac{\rho_{A}\left(x_{Q}-x_{P}\right)}{\max (|P|,|Q|)}\right)^{-J-\epsilon} \min \left[\left(\frac{|Q|}{|P|}\right)^{\frac{1+\epsilon}{2}},\left(\frac{|P|}{|Q|}\right)^{\frac{1+\epsilon}{2}+J-1}\right] .
$$

Theorem 4.1. Suppose $\alpha \in \mathbb{R}, 0<p<\infty, 0<q \leq \infty$, and $w \in A_{\infty}$. An almost diagonal operator $\mathcal{A}$ is bounded as a linear operator on $\mathbf{f}_{p}^{\alpha, q}(A, w d x)$.

Proof. Suppose first that Theorem 4.1 is true in the case $\alpha=0$. Let $\mathcal{A}$ be an almost diagonal operator on $\dot{\mathbf{f}}_{p}^{\alpha, q}$ with matrix $\left\{a_{Q P}\right\}_{Q, P}$. Let $\mathcal{B}$ be a linear operator on $\dot{\mathbf{f}}_{p}^{0, q}$ with matrix $\left\{b_{Q P}\right\}_{Q, P}$ defined by

$$
b_{Q P}=(|P| /|Q|)^{\alpha} a_{Q P} .
$$

It is easy to see that $\mathcal{B}$ satisfies the almost diagonal condition (4.1) with $\alpha=0$.

Let $\left\{s_{P}\right\}_{P} \in \dot{\mathbf{f}}_{p}^{\alpha, q}$ and define $\left\{t_{P}\right\}_{P} \in \dot{\mathbf{f}}_{p}^{0, q}$ by $t_{P}=|P|^{-\alpha} s_{P}$. By the result for $\alpha=0$, we have

$$
\begin{array}{r}
\left\|\left\{\sum_{P} a_{Q P} s_{P}\right\}_{Q}\right\|_{\dot{\mathbf{f}}_{p}^{\alpha, q}}=\left\|\left\{\sum_{P}|Q|^{-\alpha} a_{Q P} s_{P}\right\}_{Q}\right\|_{\dot{\mathbf{f}}_{p}^{0, q}}=\left\|\left\{\sum_{P} b_{Q P} t_{P}\right\}_{Q}\right\|_{\dot{\mathbf{f}}_{p}^{0, q}} \\
\leq C\left\|\left\{t_{P}\right\}_{P}\right\|_{\dot{\mathbf{f}}_{p}^{0, q}}=C\left\|\left\{s_{P}\right\}_{P}\right\|_{\dot{\mathbf{f}}_{p}^{\alpha, q}},
\end{array}
$$

which reduces the theorem to the case $\alpha=0$.

First, we consider the case $r=\min \left(p / r_{0}, q\right)>1$, which implies that $J=1$ in Definition 4.1. Let $\mathcal{A}$ be an almost diagonal operator on $\dot{\mathbf{f}}_{p}^{0, q}$ with matrix $\left\{a_{Q P}\right\}_{Q, P}$ satisfying condition (4.1). We write $\mathcal{A}=\mathcal{A}_{0}+\mathcal{A}_{1}$, with

$$
\left(\mathcal{A}_{0} s\right)_{Q}=\sum_{P \in \mathcal{Q},|P| \geq|Q|} a_{Q P} s_{P} \quad \text { and } \quad\left(\mathcal{A}_{1} s\right)_{Q}=\sum_{P \in \mathcal{Q},|P|<|Q|} a_{Q P} s_{P}
$$

for $s=\left\{s_{P}\right\}_{P} \in \dot{\mathbf{f}}_{p}^{0, q}$. For $Q \in \mathcal{Q},|Q|=|\operatorname{det} A|^{-j}$, and $x \in Q$, we have

$$
\begin{aligned}
\left|\left(\mathcal{A}_{0} s\right)_{Q}\right| & \leq C \sum_{|P| \geq|Q|} \kappa_{Q P}(\epsilon)\left|s_{P}\right| \\
& \leq C \sum_{|P| \geq|Q|}\left(\frac{|Q|}{|P|}\right)^{(1+\epsilon) / 2} \frac{\left|s_{P}\right|}{\left(1+|P|^{-1} \rho_{A}\left(x_{P}-x_{Q}\right)\right)^{1+\epsilon}} \\
& \leq C \sum_{i \leq j|P|=|\operatorname{det} A|^{-i}}\left(\frac{|Q|}{|P|}\right)^{(1+\epsilon) / 2} \frac{\left|s_{P}\right|}{\left(1+|P|^{-1} \rho_{A}\left(x_{P}-x_{Q}\right)\right)^{1+\epsilon}} \\
& \leq C \sum_{i \leq j}|\operatorname{det} A|^{(i-j)\left(\frac{1+\epsilon}{2}\right)} M_{\rho_{A}}\left(\sum_{|P|=|\operatorname{det} A|^{-i}}\left|s_{P} \chi_{P}\right|\right)(x)
\end{aligned}
$$

using Lemma 6.2, Hence, we have

$$
\left(\sum_{|Q|=|\operatorname{det} A|^{-j}}\left|\left(\mathcal{A}_{0} s\right)_{Q} \tilde{\chi}_{Q}\right|^{q}\right) \leq C\left(\sum_{i \leq j}|\operatorname{det} A|^{(i-j)\left(\frac{\epsilon}{2}\right)} M_{\rho_{A}}\left(\sum_{|P|=|\operatorname{det} A|^{-i}}\left|s_{P} \tilde{\chi}_{P}\right|\right)\right)^{q} .
$$


Therefore,

$$
\begin{aligned}
&\left\|\mathcal{A}_{0} s\right\|_{\dot{\mathbf{f}}_{p}^{0, q}} \leq C \|\left(\sum_{j \in \mathbb{Z}}(\right.\left.\left.\sum_{i \leq j}|\operatorname{det} A|^{(i-j)\left(\frac{\epsilon}{2}\right)} M_{\rho_{A}}\left(\sum_{|P|=|\operatorname{det} A|^{-i}}\left|s_{P} \tilde{\chi}_{P}\right|\right)\right)^{q}\right)^{1 / q} \|_{L^{p}(w)} \\
& \leq C C\left\|\left(\sum_{i \in \mathbb{Z}}\left(M_{\rho_{A}}\left(\sum_{|P|=|\operatorname{det} A|^{-i}}\left|s_{P} \tilde{\chi}_{P}\right|\right)\right)^{q}\right)^{1 / q}\right\|_{L^{p}(w)},
\end{aligned}
$$

by Minkowski's inequality. By the Fefferman-Stein vector-valued inequality we conclude that

$$
\left\|\mathcal{A}_{0} s\right\|_{\dot{\mathbf{f}}_{p}^{0, q}} \leq C\left\|\left(\sum_{P}\left|s_{P} \tilde{\chi}_{P}\right|^{q}\right)^{1 / q}\right\|_{L^{p}(w)}=C\|s\|_{\dot{\mathbf{f}}_{p}^{0, q}},
$$

since $w \in A_{p}$ by $p>r_{0}$. To show the corresponding estimate for $\mathcal{A}_{1}$, we apply the same argument as for $\mathcal{A}_{0}$ using the condition

$$
\kappa_{Q P}(\epsilon) \leq C\left(\frac{|P|}{|Q|}\right)^{\frac{1+\epsilon}{2}}\left(1+|Q|^{-1} \rho_{A}\left(x_{P}-x_{Q}\right)\right)^{-1-\epsilon} .
$$

Therefore, both $\mathcal{A}_{0}$ and $\mathcal{A}_{1}$ are bounded on $\dot{\mathrm{f}}_{p}^{0, q}$ and, hence, $\mathcal{A}$ is also bounded.

The case $r=\min \left(p / r_{0}, q\right) \leq 1$ is a simple consequence of the case $r>1$. Indeed, we remark that $\mathcal{A}=\left\{a_{Q P}\right\}_{Q, P}$ being almost diagonal on $\dot{\mathbf{f}}_{p}^{0, q}$, i.e., (4.1) holds for some $\epsilon>0$, is equivalent to

$$
\mathcal{A}^{\prime}=\left\{a_{Q P}^{\prime}\right\}_{Q, P}=\left\{\left|a_{Q P}\right|^{r}(|Q| /|P|)^{1 / 2-r / 2}\right\}_{Q, P}
$$

being almost diagonal on $\dot{\mathbf{f}}_{p / r}^{0, q}$, i.e., (4.1) holds for $\left\{a_{Q P}^{\prime}\right\}_{Q, P}$ and $\epsilon^{\prime}=r \epsilon$. Hence, we can pick an $\tilde{r}<r$ so close to $r$ that the almost diagonal condition (4.1) still holds with $r=\min \left(p / r_{0}, q\right)$ replaced by $\tilde{r}$. This means that $p /\left(r_{0} \tilde{r}\right)>1, q / \tilde{r}>1$, and that the matrix

$$
\tilde{\mathcal{A}}=\left\{\tilde{a}_{Q P}\right\}_{Q, P}=\left\{\left|a_{Q P}\right|^{\tilde{r}}\left(\frac{|Q|}{|P|}\right)^{1 / 2-\tilde{r} / 2}\right\}_{Q, P}
$$

satisfies the almost diagonal condition (4.1) on $\dot{\mathbf{f}}_{p / \tilde{r}}^{0, q / \tilde{r}}$ for a smaller value of $\tilde{\epsilon}$ than $\epsilon^{\prime}=r \epsilon$, since $\tilde{J}=\max \left(1, r_{0} \tilde{r} / p, \tilde{r} / q\right)=1$. Indeed, we have

$$
\left|\tilde{a}_{Q P}\right| \leq C\left(1+\frac{\rho_{A}\left(x_{Q}-x_{P}\right)}{\max (|Q|,|P|)}\right)^{-\tilde{r} / r-\tilde{r} \epsilon} \min \left[\left(\frac{|Q|}{|P|}\right)^{1 / 2+\tilde{r} \epsilon / 2},\left(\frac{|P|}{|Q|}\right)^{\tilde{r} / r-1 / 2+\tilde{r} \epsilon / 2}\right] .
$$

Given $s \in \dot{\mathbf{f}}_{p}^{0, q}$, define $t=\left\{t_{Q}\right\}_{Q}$ by $t_{Q}=|Q|^{1 / 2-\tilde{r} / 2}\left|s_{Q}\right|^{\tilde{r}}$. Then

$$
\|t\|_{\dot{\mathbf{f}}_{p / \tilde{r}}^{0, q} \tilde{r}}^{1 / \tilde{r}}=\left\|\left(\sum_{Q \in \mathcal{Q}}\left(|Q|^{1 / 2-\tilde{r} / 2}\left|s_{Q}\right|^{\tilde{r}} \tilde{\chi}_{Q}\right)^{q / \tilde{r}}\right)^{\tilde{r} / q}\right\|_{L^{p / \tilde{r}}(w)}^{1 / \tilde{r}}=\|s\|_{\dot{\mathbf{f}}_{p}^{0, q}} .
$$

By the $\tilde{r}$-inequality, we have

$$
\left|(\mathcal{A} s)_{Q}\right| \leq\left(\sum_{P}\left|a_{Q P}\right|^{\tilde{r}}\left|s_{P}\right|^{\tilde{r}}\right)^{1 / \tilde{r}}=\left(|Q|^{\tilde{r} / 2-1 / 2}\right)^{1 / \tilde{r}}\left(\sum_{P}\left|\tilde{a}_{Q P}\right|\left|t_{P}\right|\right)^{1 / \tilde{r}} .
$$

Hence,

$$
\|\mathcal{A} s\|_{\dot{\mathbf{f}}_{p}^{0, q}} \leq\left\|\tilde{\mathcal{A}}\left(\left\{\left|t_{P}\right|\right\}_{P}\right)_{Q}\right\|_{\dot{\mathbf{f}}_{p / \tilde{r}}^{0, q}}^{1 / \tilde{r}} \leq C\|t\|_{\dot{\mathbf{f}}_{p / \tilde{r}}^{0, q / \tilde{r}}}^{1 / \tilde{r}}=C\|s\|_{\dot{\mathbf{f}}_{p}^{0, q}}
$$


since $\tilde{\mathcal{A}}$ is bounded on $\dot{\mathbf{f}}_{p / \tilde{r}}^{0, q} \tilde{r}(A, w d x)$, by Theorem 4.1 in the already shown case $p / r_{0}, q>1$. This completes the proof of Theorem 4.1.

\section{Atomic And molecular decompositions}

5.1. Smooth molecules. We are ready to introduce the notions of smooth molecules adapted to anisotropic setting of expansive dilation matrices considered in this work. Our definition is motivated by smooth molecules associated with the usual dyadic dilations and studied in [27, Section 3]. However, for the sake of clarity, we have decided to use a slightly simplified version of smooth molecules, where the condition on differences of partial derivatives of the highest orders is incorporated into the corresponding decay conditions on partial derivatives of one higher order. This enables us to reduce the number of conditions defining smooth molecules in 27 from 4 to 3 . Moreover, this reduction is further justified by the observation that in the non-isotropic setting one generally needs to assume the appropriate decay and smoothness conditions of higher orders than in the isotropic setting. Indeed, in the usual dyadic situation $\zeta_{-}=\zeta_{+}=1 / n$ and Definition 5.1 is equivalent (modulo the above-mentioned reduction) to the Frazier and Jawerth notion of smooth molecules. However, if a dilation matrix $A$ is non-isotropic, then $0<\zeta_{-} \leq 1 / n \leq \zeta_{+}<1$ and we have different growth rates of a quasi-norm $\rho_{A}(x)$ in different directions. To compensate for this we ought to require more smoothness and decay conditions to have meaningful notion of smooth molecules. These are reflected in conditions (5.1) $-(5.6)$.

Definition 5.1. Suppose $\alpha \in \mathbb{R}, 0<p<\infty, 0<q \leq \infty, w \in A_{\infty}$, and $r_{0}=$ $\inf \left\{r: w \in A_{r}\right\}$. Let $J=\max \left(1, r_{0} / p, 1 / q\right)$ and $N=\max \left(\left\lfloor(J-\alpha-1) / \zeta_{-}\right\rfloor,-1\right)$.

We say that $\Psi_{Q}(x)$ is a smooth synthesis molecule for $\dot{\mathbf{F}}_{p}^{\alpha, q}\left(\mathbb{R}^{n}, A, w d x\right)$ supported near $Q \in \mathcal{Q}$ with $|Q|=|\operatorname{det} A|^{-j}$ and $j \in \mathbb{Z}$, if there exist $M>J$ such that

$$
\begin{aligned}
\left|\partial^{\gamma}\left[\Psi_{Q}\left(A^{-j} \cdot\right)\right](x)\right| & \leq \frac{|\operatorname{det} A|^{j / 2}}{\left(1+\rho_{A}\left(x-A^{j} x_{Q}\right)\right)^{M}} \quad \text { for }|\gamma| \leq\left\lfloor\alpha / \zeta_{-}\right\rfloor+1, \\
\left|\Psi_{Q}(x)\right| & \leq \frac{|\operatorname{det} A|^{j / 2}}{\left(1+\rho_{A}\left(A^{j}\left(x-x_{Q}\right)\right)\right)^{\max \left(M,(M-\alpha) \zeta_{+} / \zeta_{-}\right)}}, \\
\int x^{\gamma} \Psi_{Q}(x) d x & =0 \quad \text { for }|\gamma| \leq N .
\end{aligned}
$$

We say that a collection $\left\{\Psi_{Q}\right\}_{Q \in \mathcal{Q}}$ is a family of smooth synthesis molecules, if each $\Psi_{Q}$ is a smooth synthesis molecule supported near $Q$.

We say that $\Phi_{Q}(x)$ is a smooth analysis molecule for $\dot{\mathbf{F}}_{p}^{\alpha, q}\left(\mathbb{R}^{n}, A, w d x\right)$ supported near $Q \in \mathcal{Q}$ with $|Q|=|\operatorname{det} A|^{-j}$ and $j \in \mathbb{Z}$, if there exists $M>J$ such that

$$
\begin{aligned}
\left|\partial^{\gamma}\left[\Phi_{Q}\left(A^{-j} \cdot\right)\right](x)\right| & \leq \frac{|\operatorname{det} A|^{j / 2}}{\left(1+\rho_{A}\left(x-A^{j} x_{Q}\right)\right)^{M}} \quad \text { for }|\gamma| \leq N+1, \\
\left|\Phi_{Q}(x)\right| & \leq \frac{|\operatorname{det} A|^{j / 2}}{\left(1+\rho_{A}\left(A^{j}\left(x-x_{Q}\right)\right)\right)^{\max \left(M, 1+\alpha \zeta_{+} / \zeta_{-}+M-J\right)},} \\
\int x^{\gamma} \Phi_{Q}(x) d x & =0 \quad \text { for }|\gamma| \leq\left\lfloor\alpha / \zeta_{-}\right\rfloor .
\end{aligned}
$$

We say that $\left\{\Phi_{Q}\right\}_{Q \in \mathcal{Q}}$ is a family of smooth analysis molecules if each $\Phi_{Q}$ is a smooth analysis molecule supported near $Q$. 
The following comments may clarify the above definitions.

Remark 5.1. Whenever we talk about smooth molecules or atoms, $\Phi_{Q}$ and $\Psi_{Q}$ should be understood as some function indexed by $Q=A^{-j}\left([0,1]^{n}+k\right) \in \mathcal{Q}$, which is not necessarily equal to the usual convention $\Psi_{Q}(x)=|\operatorname{det} A|^{j / 2} \Psi\left(A^{j} x-k\right)$ used throughout Section 3. Conditions (5.1) and (5.4) should be understood as follows. Let $D_{A}$ be the dilation operator given by $D_{A} f(x)=f(A x)$. Then the left-hand side of (5.1) is simply $\left|\partial^{\gamma}\left(D_{A^{-j}} \Psi_{Q}\right)(x)\right|$ and similarly for (5.4). Moreover, to avoid any ambiguity, (5.1) and (5.4) require that $\Psi_{Q}$ and $\Phi_{Q}$ have continuous partial derivatives of order $\left\lfloor\alpha / \zeta_{-}\right\rfloor+1$ and $N+1$, respectively.

Remark 5.2. If $\alpha<0$, then the smoothness condition (5.1) is void. If $\alpha \geq 0$ is sufficiently large, say $\alpha \geq M\left(1-\zeta_{-} / \zeta_{+}\right)$, then (5.2) follows from (5.1). Additionally, if $\alpha>J-1$, then $N=-1$ and the vanishing moment condition (5.3) is void. Furthermore, if $\alpha=0$, then $N=\left\lfloor(J-1) / \zeta_{-}\right\rfloor$. If also $0<p \leq 1$ and $q \geq p / r_{0}$, then $N=\left\lfloor(1 / p-1) / \zeta_{-}\right\rfloor$, and (5.3) coincides with the vanishing moment condition for atoms in the anisotropic Hardy space $H_{A}^{p}\left(\mathbb{R}^{n}\right)$; see [6, Section 4]. On the other hand, if $\alpha=0$ and $\min \left(p / r_{0}, q\right) \geq 1$, then the conditions for smooth synthesis molecules reduce to (5.1) for $|\gamma| \leq 1$, (5.2), and (5.3) for $\gamma=0$. Similar comments are applicable for smooth analysis molecules.

The main motivation behind somewhat non-obvious orders of decay and smoothness imposed on smooth molecules is revealed in the following lemma, which is a non-isotropic variant of [27, Corollary B.3].

Lemma 5.1. Suppose $\left\{\Phi_{Q}\right\}_{Q}$ and $\left\{\Psi_{Q}\right\}_{Q}$ are families of smooth analysis and synthesis molecules for $\dot{\mathbf{F}}_{p}^{\alpha, q}$, respectively. Then the matrix $\left\{a_{Q P}\right\}$, given by $a_{Q P}=$ $\left\langle\Psi_{P}, \Phi_{Q}\right\rangle$, is almost diagonal. More precisely, there exist $C>0$ and $\epsilon>0$, such that

$$
\left|\left\langle\Psi_{P}, \Phi_{Q}\right\rangle\right| \leq C \kappa_{Q P}(\epsilon) \quad \text { for all } Q, P \in \mathcal{Q} .
$$

The elementary, but tedious proof of Lemma 5.1 can be found in Section 6 . As an immediate consequence we obtain the following two corollaries.

Corollary 5.2. Suppose $\left\{\Psi_{Q}\right\}_{Q}$ is a family of smooth synthesis molecules for $\dot{\mathbf{F}}_{p}^{\alpha, q}$ and $\varphi \in \mathcal{S}\left(\mathbb{R}^{n}\right)$ with $0 \notin \operatorname{supp} \hat{\varphi}$. Then the matrix $\left\{a_{Q P}\right\}$, given by $a_{Q P}=\left\langle\Psi_{P}, \varphi_{Q}\right\rangle$, is almost diagonal.

Corollary 5.3. Suppose $\left\{\Phi_{Q}\right\}_{Q}$ is a family of smooth analysis molecules for $\dot{\mathbf{F}}_{p}^{\alpha, q}$ and $\psi \in \mathcal{S}\left(\mathbb{R}^{n}\right)$ with $0 \notin \operatorname{supp} \hat{\psi}$. Then the matrix $\left\{a_{Q P}\right\}$, given by $a_{Q P}=\left\langle\psi_{P}, \Phi_{Q}\right\rangle$, is almost diagonal.

We will also need the following elementary result, which provides an approximation of smooth molecules by elements of the Schwartz class $\mathcal{S}$.

Lemma 5.4. Suppose that $\Phi$ is a smooth analysis (or synthesis) molecule supported near $Q \in \mathcal{Q}$. Then there exists a sequence $\left\{\phi_{k}\right\}_{k=1}^{\infty} \subset \mathcal{S}$ and $c>0$ such that $c \phi_{k}$ is a smooth analysis (or synthesis) molecule supported near $Q$ for every $k$, and $\phi_{k}(x) \rightarrow \Phi(x)$ uniformly on $\mathbb{R}^{n}$ as $k \rightarrow \infty$.

Proof. Using translations and dilations, we may assume that $Q=[0,1]^{n}$. Indeed, if $\Phi$ is a smooth molecule supported near $Q=A^{-j}\left([0,1]^{n}+k\right)$, then it is not hard to check that $|\operatorname{det} A|^{-j / 2} T_{-k} D_{A^{-j}} \Phi$ is a smooth molecule supported near $[0,1]^{n}$. 
We will present the argument for a smooth analysis molecule $\Phi$; the other case is identical.

First, we will show that $\Phi$ can be approximated by a sequence $\left\{\phi_{k}\right\}_{k=1}^{\infty} \subset C^{\infty}$. Let $h$ be a $C^{\infty}$ function such that $h(x) \geq 0$ for all $x \in \mathbb{R}^{n}$, supp $h \subset B(0,1)$, and $\int h=1$. Suppose that $\Phi$ is a smooth analysis molecule for some $Q \in \mathcal{Q}$; the other case is analogous. Then, we claim that $\phi_{k}=\Phi * h_{k}$, where $h_{k}(x)=2^{n k} h\left(2^{k} x\right)$, does the job. Indeed, using $\partial^{\gamma} \phi_{k}=\left(\partial^{\gamma} \Phi\right) * h_{k}$, supp $h_{k} \subset A^{-k} B(0,1)$, and $\int h_{k}=1$, it is not hard to check that $c \phi_{k}$ satisfies (5.4) and (5.5) for sufficiently small $c>0$. It is also obvious that $\phi_{k} \in C^{\infty}$ satisfies (5.6) and $\phi_{k}(x) \rightarrow \Phi(x)$ uniformly as $k \rightarrow \infty$.

Therefore, by the diagonal argument, we can assume that $\Phi \in C^{\infty}$. Let $g$ be a $C^{\infty}$ function such that $g(x)=1$ for $x \in B(0,1)$, and $\operatorname{supp} g \subset B(0,2)$. For every multi-index $\beta$, let $\phi^{\beta} \in \mathcal{S}$ be such that $\int x^{\gamma} \phi^{\beta}(x) d x=\delta_{\beta, \gamma}$. For example, take $\widehat{\phi^{\beta}}(\xi)=(-i)^{|\beta|} \xi^{\beta} g(\xi)$. Then, we claim that

$$
\phi_{k}=\Phi g_{-k}-\sum_{|\beta| \leq L} c_{k}^{\beta} \phi^{\beta}, \quad \text { where } c_{k}^{\beta}=\int x^{\beta} \Phi(x) g_{-k}(x) d x,
$$

$L=\left\lfloor\alpha / \zeta_{-}\right\rfloor$and $g_{-k}(x)=2^{-n k} g\left(2^{-k} x\right)$ does the job. Indeed, it is clear that $c_{k}^{\beta} \rightarrow 0$ as $k \rightarrow \infty$. Since each $\phi^{\beta} \in \mathcal{S}$, then by the product and chain rules $c \phi_{k}$ satisfies (5.4) and (5.5) for sufficiently small $c>0$. Moreover, by our choice, $\phi_{k}$ has vanishing moments up to order $L, \phi_{k} \in \mathcal{S}$, and $\phi_{k}(x) \rightarrow \Phi(x)$ uniformly as $k \rightarrow \infty$. This completes the proof of Lemma 5.4 .

5.2. Smooth molecular decompositions. We are now ready to show generalizations of Theorem 3.5 in the situation when the usual wavelet families of translates and dilates $\left\{\varphi_{Q}\right\}_{Q \in \mathcal{Q}}$ and $\left\{\psi_{Q}\right\}_{Q \in \mathcal{Q}}$ are replaced by families of smooth analysis $\left\{\Phi_{Q}\right\}_{Q \in \mathcal{Q}}$ and synthesis molecules $\left\{\Psi_{Q}\right\}_{Q \in \mathcal{Q}}$.

Theorem 5.5 (Smooth molecular synthesis). Suppose $A$ is an expansive matrix and $w \in A_{\infty}$. There exists a constant $C>0$, such that if $f=\sum_{Q \in \mathcal{Q}} s_{Q} \Psi_{Q}$, where $\left\{\Psi_{Q}\right\}_{Q}$ is a family of smooth synthesis molecules for $\dot{\mathbf{F}}_{p}^{\alpha, q}\left(\mathbb{R}^{n}, A, w d x\right)$, then

$$
\|f\|_{\dot{\mathbf{F}}_{p}^{\alpha, q}} \leq C\left\|\left\{s_{Q}\right\}_{Q}\right\|_{\dot{\mathbf{f}}_{p}^{\alpha, q}} \quad \text { for all }\left\{s_{Q}\right\}_{Q} \in \dot{\mathbf{f}}_{p}^{\alpha, q} .
$$

Proof. By Lemma 2.8, we can write $\Psi_{P}=\sum_{Q}\left\langle\Psi_{P}, \varphi_{Q}\right\rangle \psi_{Q}$ with the convergence in $\mathcal{S}^{\prime} / \mathcal{P}$. By Theorem 4.1 and Corollary 5.2, $\mathcal{A}$ given by the matrix $\left\{a_{Q P}\right\}_{Q, P}=$ $\left\{\left\langle\Psi_{P}, \varphi_{Q}\right\rangle\right\}_{Q, P}$ is a bounded operator on $\dot{\mathbf{f}}_{p}^{\alpha, q}(A, w d x)$. Since

$$
\mathrm{T}_{\psi} \mathcal{A} s=\sum_{Q} \sum_{P} a_{Q P} s_{P} \psi_{Q}=\sum_{P} s_{P} \sum_{Q}\left\langle\Psi_{P}, \varphi_{Q}\right\rangle \psi_{Q}=\sum_{P} s_{P} \Psi_{P}=f,
$$

then by Theorem 3.5 ,

$$
\|f\|_{\dot{\mathbf{F}}_{p}^{\alpha, q}\left(\mathbb{R}^{n}, A, w d x\right)}=\left\|\mathrm{T}_{\psi} \mathcal{A} s\right\|_{\dot{\mathbf{F}}_{p}^{\alpha, q}\left(\mathbb{R}^{n}, A, w d x\right)} \leq C\|\mathcal{A} s\|_{\dot{\mathbf{f}}_{p}^{\alpha, q}(A, w d x)} \leq C\|s\|_{\dot{\mathbf{f}}_{p}^{\alpha, q}(A, w d x)} .
$$

Theorem 5.6 (Smooth molecular analysis). Suppose $A$ is an expansive matrix and $w \in A_{\infty}$. There exists a constant $C>0$, such that if $\left\{\Phi_{Q}\right\}_{Q}$ is a family of smooth analysis molecules, then

$$
\left\|\left\{\left\langle f, \Phi_{Q}\right\rangle\right\}_{Q}\right\|_{\dot{\mathbf{f}}_{p}^{\alpha, q}} \leq C\|f\|_{\dot{\mathbf{F}}_{p}^{\alpha, q}} \quad \text { for all } f \in \dot{\mathbf{F}}_{p}^{\alpha, q}\left(\mathbb{R}^{n}, A, w d x\right) .
$$


The main technical difficulty in the proof of the above theorem is to justify the meaningfulness of the pairing $\left\langle f, \Phi_{Q}\right\rangle$. Indeed, $f \in \dot{\mathbf{F}}_{p}^{\alpha, q}$ is an equivalence class in $\mathcal{S}^{\prime} / \mathcal{P}$, and $\Phi_{Q}$ may not even belong to $\mathcal{S}$, and consequently, it may not be clear how to understand $\left\langle f, \Phi_{Q}\right\rangle$. More than that, even when $\Phi_{Q}$ happens to be in $\mathcal{S}$, then $\left\langle f, \Phi_{Q}\right\rangle$ may still not be well defined, since it is necessary to choose an appropriate representative of $f$ in $\mathcal{S}^{\prime}$ in its equivalence class in $\mathcal{S}^{\prime} / \mathcal{P}$ for $\left\langle f, \Phi_{Q}\right\rangle$ to be understood as the usual pairing of a tempered distribution $f \in \mathcal{S}^{\prime}$ with a test function $\Phi_{Q} \in \mathcal{S}$. Therefore, we need a precise pairing procedure provided by Lemma 5.7

Lemma 5.7. Suppose $f \in \dot{\mathbf{F}}_{p}^{\alpha, q}\left(\mathbb{R}^{n}, A, w d x\right)$ and $\Phi_{Q}$ is a smooth analysis molecule for $\dot{\mathbf{F}}_{p}^{\alpha, q}\left(\mathbb{R}^{n}, A, w d x\right)$ supported near $Q \in \mathcal{Q}$. Then for any $\varphi, \psi \in \mathcal{S}\left(\mathbb{R}^{n}\right)$ satisfying (2.9) and (2.10), the series

$$
\left\langle f, \Phi_{Q}\right\rangle:=\sum_{j \in \mathbb{Z}}\left\langle\tilde{\varphi}_{j} * \psi_{j} * f, \Phi_{Q}\right\rangle=\sum_{P \in \mathcal{Q}}\left\langle f, \varphi_{P}\right\rangle\left\langle\psi_{P}, \Phi_{Q}\right\rangle
$$

converges absolutely and its value is independent of the choice of $\varphi$ and $\psi$ satisfying (2.9) and (2.10).

Proof. First, note that for any $f \in \dot{\mathbf{F}}_{p}^{\alpha, q}$, there exists a matrix $\left\{b_{Q P}\right\}_{Q, P \in \mathcal{Q}}$ such that $b_{Q P} \geq 0$ and

$$
\left|\left\langle f, \varphi_{P}\right\rangle\right|\left|\left\langle\psi_{P}, \phi\right\rangle\right| \leq b_{Q P} \quad \text { and } \quad \sum_{P} b_{Q P}<\infty,
$$

whenever $\phi$ is a smooth analysis molecule supported near $Q$. Indeed, by Corollary 5.3, there exist $C>0$ and $\epsilon>0$ such that $\left|\left\langle\psi_{P}, \phi\right\rangle\right| \leq C \kappa_{Q P}(\epsilon)$, and hence $b_{Q P}=$ $C\left|\left\langle f, \varphi_{P}\right\rangle\right| \kappa_{Q P}(\epsilon)$ does the job. Moreover, by Theorem 3.5. $\left\{\left\langle f, \varphi_{P}\right\rangle\right\}_{P} \in \dot{\mathbf{f}}_{p}^{\alpha, q}$, and hence by Theorem $4.1 \sum_{P} b_{Q P}<\infty$. This shows the absolute convergence of the series in (5.7).

To show independence of the choice of $\varphi$ and $\psi$, let $\left\{\phi_{l}\right\}_{l=1}^{\infty} \subset \mathcal{S}$ be the sequence of (constant multiples of) smooth analysis molecules supported near $Q$ and converging uniformly to $\Phi_{Q}$ guaranteed by Lemma 5.4. By Proposition 3.8 and Corollary 3.9. there exists a sequence of polynomials $\left\{P_{k}\right\}_{k=1}^{\infty}$ with $\operatorname{deg} P_{k} \leq L=\left\lfloor\alpha / \zeta_{-}\right\rfloor$such that $\sum_{j=-k}^{\infty} \tilde{\varphi}_{j} * \psi_{j} * f+P_{k}$ converges in $\mathcal{S}^{\prime}$ as $k \rightarrow \infty$. Therefore, for each $l$, we can define

$$
\begin{array}{r}
\left\langle f, \phi_{l}\right\rangle:=\left\langle\lim _{k \rightarrow \infty} \sum_{j=-k}^{\infty} \tilde{\varphi}_{j} * \psi_{j} * f+P_{k}, \phi_{l}\right\rangle=\lim _{k \rightarrow \infty} \sum_{j=-k}^{\infty}\left\langle\tilde{\varphi}_{j} * \psi_{j} * f, \phi_{l}\right\rangle \\
=\lim _{k \rightarrow \infty} \sum_{P \in \mathcal{Q},|P| \geq|\operatorname{det} A|^{-k}}\left\langle f, \varphi_{P}\right\rangle\left\langle\psi_{P}, \phi_{l}\right\rangle=\sum_{P \in \mathcal{Q}}\left\langle f, \varphi_{P}\right\rangle\left\langle\psi_{P}, \phi_{l}\right\rangle,
\end{array}
$$

since the above series converges absolutely by (5.8). Moreover, by (3.11) in Proposition 3.8 and (5.6), this definition does not depend on the choice of $\varphi$ and $\psi$. Since $\left\langle\psi_{P}, \phi_{l}\right\rangle \rightarrow\left\langle\psi_{P}, \Phi_{Q}\right\rangle$ as $l \rightarrow \infty$, by (5.7) and the Lebesgue Dominated Convergence Theorem,

$$
\sum_{P \in \mathcal{Q}}\left\langle f, \varphi_{P}\right\rangle\left\langle\psi_{P}, \phi_{l}\right\rangle \rightarrow \sum_{P \in \mathcal{Q}}\left\langle f, \varphi_{P}\right\rangle\left\langle\psi_{P}, \Phi_{Q}\right\rangle \quad \text { as } l \rightarrow \infty .
$$

By the above reasoning, this limit is independent of $\varphi$ and $\psi$ satisfying (2.9) and (2.10). This shows that $\left\langle f, \Phi_{Q}\right\rangle$ is well defined by (5.7) and completes the proof of Lemma 5.7 
Proof of Theorem [5.6. After hard work in Lemma 5.7, the proof is now completely trivial. Recall that by Lemma 5.7 .

$$
\left\langle f, \Phi_{Q}\right\rangle:=\sum_{P}\left\langle f, \varphi_{P}\right\rangle\left\langle\psi_{P}, \Phi_{Q}\right\rangle .
$$

By Theorem 4.1 and Corollary 5.2 , the operator $\mathcal{A}$ given by the matrix $\left\{a_{Q P}\right\}_{Q, P}=$ $\left\{\left\langle\psi_{P}, \Phi_{Q}\right\rangle\right\}_{Q, P}$ is bounded on $\dot{\mathbf{f}}_{p}^{\alpha, q}(A, w d x)$. Since $\left\langle f, \Phi_{Q}\right\rangle=\sum_{P}\left\langle f, \varphi_{P}\right\rangle a_{Q P}$, by Theorem 3.5, we have

$$
\left\|\left\{\left\langle f, \Phi_{Q}\right\rangle\right\}\right\|_{\dot{\mathbf{f}}_{p}^{\alpha, q}(A, w d x)}=\left\|\mathcal{A} S_{\varphi} f\right\|_{\dot{\mathbf{f}}_{p}^{\alpha, q}(A, w d x)} \leq C\|f\|_{\dot{\mathbf{F}}_{p}^{\alpha, q}\left(\mathbb{R}^{n}, A, w d x\right)} .
$$

5.3. Smooth atomic decompositions. In this subsection we show that the elements of $\dot{\mathbf{F}}_{p}^{\alpha, q}\left(\mathbb{R}^{n}, A, w d x\right)$ admit smooth atomic decompositions.

Definition 5.2. A function $a_{Q}(x)$ is said to be a smooth atom supported near a cube $Q=Q_{j, k}=A^{-j}\left([0,1]^{n}+k\right) \in \mathcal{Q}$ if it satisfies

$$
\operatorname{supp} a_{Q} \subset A^{-j}\left(\left[-\delta_{0}, 1+\delta_{0}\right]^{n}+k\right),
$$

where $\delta_{0}>0$ is some fixed constant, and

$$
\begin{aligned}
\left|\partial^{\gamma}\left[a_{Q}\left(A^{-j} \cdot\right)\right](x)\right| \leq|Q|^{-1 / 2} & \text { for }|\gamma| \leq \tilde{K}, \\
\int_{\mathbb{R}^{n}} x^{\gamma} a_{Q}(x) d x=0 & \text { for }|\gamma| \leq \tilde{N},
\end{aligned}
$$

where $\tilde{N} \geq N$ is the same as in Definition 5.1 and $\tilde{K} \geq \max \left(\left\lfloor\alpha / \zeta_{-}\right\rfloor+1,0\right)$. Recall that

$N=\max \left(\left\lfloor(J-\alpha-1) / \zeta_{-}\right\rfloor,-1\right) \quad$ where $J=\max \left(1, r_{0} / p, 1 / q\right), r_{0}=\inf \left\{r: w \in A_{r}\right\}$.

When more emphasis is needed, we say that $a_{Q}$ is a $(\tilde{K}, \tilde{N})$-smooth atom.

We say that $\left\{a_{Q}\right\}_{Q \in \mathcal{Q}}$ is a family of smooth atoms, if each function $a_{Q}$ is a smooth atom supported near $Q$.

Remark 5.3. We remark that every smooth atom $a_{Q}$ is always some fixed constant multiple of a smooth synthesis molecule supported near $Q$. Moreover, this constant multiple depends only on $\delta_{0}>0$, which controls the relative size of the support of $a_{Q}$. Indeed, it is clear that the support condition (5.9) together with (5.10) imply the decay condition (5.2) of $a_{Q}$ and its partial derivatives (5.1) for any value of $M>J$.

Theorem 5.8 (Smooth atomic decomposition). Suppose $A$ is an expansive matrix, $\alpha \in \mathbb{R}, 0<p<\infty, 0<q \leq \infty$, and $w \in A_{\infty}$. For any $f \in \dot{\mathbf{F}}_{p}^{\alpha, q}$ there exists a family of smooth atoms $\left\{a_{Q}\right\}$ and a sequence of coefficients $s=\left\{s_{Q}\right\} \in \dot{\mathbf{f}}_{p}^{\alpha, q}$, such that

$$
f=\sum_{Q \in \mathcal{Q}} s_{Q} a_{Q} \quad \text { and } \quad\|s\|_{\dot{\mathbf{f}}_{p}^{\alpha, q}} \leq C\|f\|_{\dot{\mathbf{F}}_{p}^{\alpha, q}}
$$

where the above series converges unconditionally in $\dot{\mathbf{F}}_{p}^{\alpha, q}$. Conversely, for any family of smooth atoms $\left\{a_{Q}\right\}$,

$$
\left\|\sum_{Q} s_{Q} a_{Q}\right\|_{\dot{\mathbf{F}}_{p}^{\alpha, q}} \leq C\|s\|_{\dot{\mathbf{f}}_{p}^{\alpha, q}}
$$


Proof. The converse direction (5.13) follows immediately from Theorem 5.5 and Remark 5.3. Let $\theta \in \mathcal{S}$ be such that $\operatorname{supp} \theta \subset B\left(0, \delta_{0}\right)$, and

$$
\begin{aligned}
\int x^{\gamma} \theta(x) d x=0 & \text { for all }|\gamma| \leq \tilde{N}, \\
|\hat{\theta}(\xi)| \geq c>0 & \text { for all }(2|| A||)^{-1} \leq|\xi| \leq 1 .
\end{aligned}
$$

It is easy to construct such $\theta$; see [25, Theorem 2.6]. Indeed, let $\Theta \in \mathcal{S}$ be a radial function satisfying supp $\Theta \subset B(0,1)$ and $\hat{\Theta}(0)=1$. Then for sufficiently small $0<$ $\delta<\delta_{0}, \theta=(-\Delta)^{\tilde{N}}\left(\delta^{-n} \Theta(\cdot / \delta)\right)$ satisfies (5.14) and (5.15), since $\hat{\theta}(\xi)=|\xi|^{2 \tilde{N}} \hat{\Theta}(\delta \xi)$. Let $\eta \in \mathcal{S}$ be such that $\hat{\eta}(\xi) \neq 0 \Longleftrightarrow(2|| A||)^{-1}<|\xi|<1$. In addition, by (5.15) we can modify the phase of $\hat{\eta}(\xi)$ such that $\hat{\eta}(\xi) \hat{\theta}(\xi) \geq 0$ for all $\xi \in \mathbb{R}^{n}$. Let $h(\xi)=\sum_{j \in \mathbb{Z}} \hat{\eta}\left(\left(A^{*}\right)^{j} \xi\right) \hat{\theta}\left(\left(A^{*}\right)^{j} \xi\right)$. Finally, let $\varphi$ be given by $\hat{\varphi}(\xi)=\hat{\eta}(\xi) / h(\xi)$. Then, as in the proof of Lemma 3.6. one can show that $\varphi \in \mathcal{S}$ satisfies (3.2), (3.3), and

$$
\sum_{j \in \mathbb{Z}} \hat{\varphi}\left(\left(A^{*}\right)^{j} \xi\right) \hat{\theta}\left(\left(A^{*}\right)^{j} \xi\right)=1 \quad \text { for all } \xi \in \mathbb{R}^{n} \backslash\{0\} .
$$

Therefore, by Lemma 2.6 we can expand $f \in \dot{\mathbf{F}}_{p}^{\alpha, q}$ as

$$
f=\sum_{j \in \mathbb{Z}} \theta_{j} * \varphi_{j} * f
$$

where the equality and convergence is in $\mathcal{S}^{\prime} / \mathcal{P}$. Then, we can decompose the first convolution to obtain the expansion of $f$

$$
f(x)=\sum_{j \in \mathbb{Z}} \sum_{Q \in \mathcal{Q},|Q|=\mid \operatorname{det}} \int_{\left.A\right|^{-j}} \theta_{Q}(x-y)\left(\varphi_{j} * f\right)(y) d y .
$$

At this moment, it may not be obvious that the above series converges in $\mathcal{S}^{\prime} / \mathcal{P}$. However, if it does, then clearly this series must converge to $f$ in $\mathcal{S}^{\prime} / \mathcal{P}$. Next, for $Q \in \mathcal{Q}$ with $|Q|=|\operatorname{det} A|^{-j}$, we define

$$
s_{Q}=|Q|^{1 / 2} \sup _{y \in Q}\left|\left(\varphi_{j} * f\right)(y)\right| .
$$

It follows from the proof of the boundedness of $S_{\varphi}$ in Theorem 3.5 that $s=\left\{s_{Q}\right\}_{Q} \in$ $\dot{\mathbf{f}}_{p}^{\alpha, q}$ and $\|s\|_{\dot{\mathbf{f}}_{p}^{\alpha, q}} \leq C\|f\|_{\dot{\mathbf{F}}_{p}^{\alpha, q}}$. We also define

$$
a_{Q}(x)=s_{Q}^{-1} \int_{Q} \theta_{j}(x-y)\left(\varphi_{j} * f\right)(y) d y, \quad \text { if } s_{Q} \neq 0,
$$

and $a_{Q} \equiv 0$ if $s_{Q}=0$. Therefore, we can rewrite (5.16) as $f=\sum_{Q \in \mathcal{Q}} s_{Q} a_{Q}$.

To guarantee that this series converges in $\dot{\mathbf{F}}_{p}^{\alpha, q}$, by Theorem 5.5 and Remark 5.3 . it suffices to verify that each $a_{Q}$ is a smooth atom. It is immediate that $a_{Q}$ satisfies (5.9) and (5.11). Finally, to check (5.10), for any multi-index $\gamma$ and $|Q|=|\operatorname{det} A|^{-j}$, we have

$$
\begin{aligned}
\left|\partial^{\gamma}\left[a_{Q}\left(A^{-j} \cdot\right)\right](x)\right| & \leq s_{Q}^{-1}|\operatorname{det} A|^{j} \int_{Q}\left|\partial^{\gamma} \theta\left(x-A^{j} y\right)\right|\left|\left(\varphi_{j} * f\right)(y)\right| d y \\
& \leq|\operatorname{det} A|^{j} s_{Q}^{-1} \sup _{y \in Q}\left|\left(\varphi_{j} * f\right)(y)\right| \int_{Q}\left|\partial^{\gamma} \theta\left(x-A^{j} y\right)\right| d y \\
& \leq|Q|^{-1 / 2} \int_{\mathbb{R}^{n}}\left|\partial^{\gamma} \theta(y)\right| d y \leq C_{\gamma}|Q|^{-1 / 2} .
\end{aligned}
$$


Finally, by re-normalizing $\left\{a_{Q}\right\}$ and $\left\{s_{Q}\right\}$ by the constant factor $\sup _{|\gamma| \leq \tilde{K}} C_{\gamma},\left\{a_{Q}\right\}$ is the required family of smooth atoms. This completes the proof of Theorem 5.8 .

5.4. Atomic and molecular decompositions of $\mathbf{F}_{p}^{\alpha, q}$. After some necessary modifications, all results about smooth atomic and molecular decompositions for the homogeneous case can be generalized to the inhomogeneous case. For the sake of completeness, we present the inhomogeneous versions of Theorem 5.5. Theorem 5.6. and Theorem 5.8 ,

Definition 5.3. Suppose $\alpha \in \mathbb{R}, 0<p<\infty, 0<q \leq \infty, w \in A_{\infty}$, and $r_{0}=$ $\inf \left\{r: w \in A_{r}\right\}$. Let $\mathcal{Q}_{0}=\{Q \in \mathcal{Q}:|Q| \leq 1\}$.

We say that $\Psi_{Q}(x)$ is an inhomogeneous smooth synthesis molecule for $\mathbf{F}_{p}^{\alpha, q}$ supported near $Q \in \mathcal{Q}_{0}$ if it satisfies (5.1) (5.3) if $|Q|<1$, and (5.1) only if $|Q|=1$. Hence, we do not assume that $\Psi_{Q}$ has any vanishing moments if $|Q|=1$. A collection $\left\{\Psi_{Q}\right\}_{Q \in \mathcal{Q}_{0}}$ is a family of inhomogeneous smooth synthesis molecules if each $\Psi_{Q}$ is a smooth synthesis molecule supported near $Q$.

We say that $\Phi_{Q}(x)$ is an inhomogeneous smooth analysis molecule for $\mathbf{F}_{p}^{\alpha, q}$ supported near $Q \in \mathcal{Q}_{0}$ if it satisfies (5.4) (5.6) if $|Q|<1$, and (5.4) only if $|Q|=1$. A collection $\left\{\Phi_{Q}\right\}_{Q \in \mathcal{Q}_{0}}$ is a family of inhomogeneous smooth analysis molecules if each $\Phi_{Q}$ is a smooth analysis molecule supported near $Q$.

A matrix $\left\{a_{P Q}\right\}_{P, Q \in \mathcal{Q}_{0}}$ is an inhomogeneous almost diagonal matrix for $\mathbf{f}_{p}^{\alpha, q}$ if there exists an $\epsilon>0$ such that

$$
\sup _{P, Q \in \mathcal{Q}_{0}}\left|a_{Q P}\right| / \kappa_{Q P}(\epsilon)<\infty .
$$

It is clear that an operator $\mathcal{A}$ on $\mathbf{f}_{p}^{\alpha, q}$ given by an almost diagonal matrix is bounded on $\mathbf{f}_{p}^{\alpha, q}$. This follows immediately from Theorem 4.1 and the observation that $\mathbf{f}_{p}^{\alpha, q} \hookrightarrow \dot{\mathbf{f}}_{p}^{\alpha, q}$ is an isometric embedding.

Suppose $\left\{\Psi_{Q}\right\}$ and $\left\{\Phi_{Q}\right\}$ are families of inhomogeneous smooth synthesis and analysis molecules, respectively. Then the inhomogeneous analogue of Lemma 5.1 holds, i.e., the matrix $\left\{a_{P Q}\right\}_{P, Q}=\left\{\left\langle\Psi_{P}, \Phi_{Q}\right\rangle\right\}_{P, Q}$ is almost diagonal on $\mathbf{f}_{p}^{\alpha, q}$. The proof of this fact is a slight modification of the homogeneous case. Indeed, the vanishing conditions for $\Psi_{Q}$ and the additional decay in the case $\alpha<0$ are only used in cases III and IV of Lemma 5.1, i.e., when applying Lemma 6.3 for $h=\Psi_{P}$ and $|P|<|Q|$. This never happens in the inhomogeneous case if $|P|=1$. Similar observation holds for $\Phi_{Q}$ if $|Q|=1$. The remaining estimate for $\left\langle\Psi_{P}, \Phi_{Q}\right\rangle$ for $|P|=|Q|=1$ is a consequence of Lemma 6.4. Hence, the inhomogeneous analogue of Lemma 5.1 is true.

As a consequence, we have the following analogues for the corresponding results for the homogeneous case.

Theorem 5.9 (Inhomogeneous smooth molecular synthesis). Suppose $A$ is an expansive matrix and $w \in A_{\infty}$. There exists a constant $C>0$, such that if $f=\sum_{Q \in \mathcal{Q}_{0}} s_{Q} \Psi_{Q}$, where $\left\{\Psi_{Q}\right\}_{Q}$ is a family of inhomogeneous smooth synthesis molecules for $\mathbf{F}_{p}^{\alpha, q}\left(\mathbb{R}^{n}, A\right.$, wdx $)$, then

$$
\|f\|_{\mathbf{F}_{p}^{\alpha, q}} \leq C\left\|\left\{s_{Q}\right\}_{Q}\right\|_{\mathbf{f}_{p}^{\alpha, q}} \quad \text { for all }\left\{s_{Q}\right\}_{Q} \in \mathbf{f}_{p}^{\alpha, q} .
$$

Theorem 5.10 (Inhomogeneous smooth molecular analysis). Suppose $A$ is an expansive matrix and $w \in A_{\infty}$. There exists a constant $C>0$, such that if $\left\{\Phi_{Q}\right\}_{Q}$ is 
a family of inhomogeneous smooth analysis molecules, then

$$
\left\|\left\{\left\langle f, \Phi_{Q}\right\rangle\right\}_{Q \in \mathcal{Q}_{0}}\right\|_{\mathbf{f}_{p}^{\alpha, q}} \leq C\|f\|_{\mathbf{F}_{p}^{\alpha, q}} \quad \text { for all } f \in \mathbf{F}_{p}^{\alpha, q}\left(\mathbb{R}^{n}, A, w d x\right) .
$$

For the analogue of smooth atomic decomposition we need the following definition.

Definition 5.4. A function $a_{Q}(x)$ is said to be an inhomogeneous smooth atom supported near a cube $Q \in \mathcal{Q}_{0}$ if it satisfies (5.9), (5.10), and (5.11) if $|Q|<1$ and (5.9) and (5.10) only if $|Q|=1$. We say that $\left\{a_{Q}\right\}_{Q \in \mathcal{Q}_{0}}$ is a family of inhomogeneous smooth atoms if each function $a_{Q}$ is a smooth atom supported near $Q$.

Theorem 5.11 (Inhomogeneous smooth atomic decomposition). Suppose $A$ is an expansive matrix and $w \in A_{\infty}$. For any $f \in \mathbf{F}_{p}^{\alpha, q}$ there exists a family of inhomogeneous smooth atoms $\left\{a_{Q}\right\}$ and a sequence of coefficients $s=\left\{s_{Q}\right\} \in \mathbf{f}_{p}^{\alpha, q}$, such that

$$
f=\sum_{Q \in \mathcal{Q}_{0}} s_{Q} a_{Q} \quad \text { and } \quad\|s\|_{\mathbf{f}_{p}^{\alpha, q}} \leq C\|f\|_{\mathbf{F}_{p}^{\alpha, q}},
$$

where the above series converges unconditionally in $\mathbf{F}_{p}^{\alpha, q}$. Conversely, for any family of inhomogeneous smooth atoms $\left\{a_{Q}\right\}$,

$$
\left\|\sum_{Q \in \mathcal{Q}_{0}} s_{Q} a_{Q}\right\|_{\mathbf{F}_{p}^{\alpha, q}} \leq C\|s\|_{\mathbf{f}_{p}^{\alpha, q}}
$$

The proof of Theorem 5.11 is a direct modification of Theorem 5.8 Indeed, let $\theta, \Theta \in \mathcal{S}$ be such that $\operatorname{supp} \theta, \operatorname{supp} \Theta \subset B\left(0, \delta_{0}\right),|\hat{\Theta}(\xi)| \geq c>0$ for $|\xi| \leq 1$, and $\theta$ satisfies (5.14) and (5.15). Then, one can show that there exists a pair $\Phi, \varphi \in \mathcal{S}$ satisfying (3.2), (3.18), (3.19) and

$$
\hat{\Phi}(\xi) \hat{\Theta}(\xi)+\sum_{j=1}^{\infty} \hat{\varphi}\left(\left(A^{*}\right)^{-j} \xi\right) \hat{\theta}\left(\left(A^{*}\right)^{-j} \xi\right)=1 \quad \text { for all } \xi \in \mathbb{R}^{n} .
$$

Using the representation formula

$$
f=\Theta * \Phi * f+\sum_{j \geq 1} \theta_{j} * \varphi_{j} * f,
$$

the rest of the proof of Theorem 5.11 is analogous to that of Theorem 5.8

\section{Proofs of auXiLiary Results}

6.1. Proof of Proposition 2.7. To show Proposition 2.7 we will need the following lemma.

Lemma 6.1. Given an integer $d \geq 0$, define

$$
\mathcal{S}_{d}\left(\mathbb{R}^{n}\right)=\left\{\phi \in \mathcal{S}\left(\mathbb{R}^{n}\right): \partial^{\alpha} \phi(0)=0 \quad \text { for }|\alpha| \leq d\right\} .
$$

Then there exists a collection $\left\{T_{\gamma}\right\}_{|\gamma|=d+1}$ of continuous linear maps $T_{\gamma}: \mathcal{S}_{d}\left(\mathbb{R}^{n}\right) \rightarrow$ $\mathcal{S}\left(\mathbb{R}^{n}\right)$ such that any $\phi \in \mathcal{S}_{d}\left(\mathbb{R}^{n}\right)$ can be decomposed as

$$
\phi(x)=\sum_{|\gamma|=d+1} x^{\gamma} T_{\gamma} \phi(x) \quad \text { for all } x \in \mathbb{R}^{n} .
$$


Sketch of the proof. Lemma 6.1 is trivial in one dimension, since $T_{d+1}: \mathcal{S}_{d}(\mathbb{R}) \rightarrow$ $\mathcal{S}(\mathbb{R})$ given by

$$
T_{d} \phi(x)= \begin{cases}\frac{\phi(x)}{x^{d+1}}, & x \neq 0, \\ \frac{\phi^{(d+1)}(0)}{(d+1) !}, & x=0,\end{cases}
$$

is a continuous linear map. Indeed, this is a consequence of the Taylor remainder theorem.

To show Lemma 6.1 in higher dimensions we will proceed by induction. Assume it is true in all dimension $<n$ for all $d \geq 0$.

Pick any $\phi \in \mathcal{S}_{d}\left(\mathbb{R}^{n}\right)$. For any $\left(x_{2}, \ldots, x_{n}\right) \in \mathbb{R}^{n}$ we expand $\phi$ in the Taylor polynomial of order $d$ at the point $\left(0, x_{2}, \ldots, x_{n}\right)$, but only in $x_{1}$ variable

$$
\phi\left(x_{1}, \ldots, x_{n}\right)=\sum_{j=0}^{d} \frac{\partial_{x_{1}}^{j} \phi\left(0, x_{2}, \ldots, x_{n}\right)}{j !}\left(x_{1}\right)^{j}+R_{\phi}\left(x_{1}, x_{2}, \ldots, x_{n}\right),
$$

where the remainder satisfies $R_{\phi}\left(x_{1}, x_{2}, \ldots, x_{n}\right)=O\left(\left|x_{1}\right|^{d+1}\right)$ as $\left|x_{1}\right| \rightarrow 0$. Let $g$ be a fixed $C^{\infty}$ function on $\mathbb{R}$ such that $g(x)=1$ for all $x \in[-1,1]$ and supp $g \subset(-2,2)$. We define the map $T_{\gamma_{0}}: \mathcal{S}_{d}\left(\mathbb{R}^{n}\right) \rightarrow \mathcal{S}\left(\mathbb{R}^{n}\right)$, where $\gamma_{0}=(d+1,0, \ldots, 0)$, by

$$
T_{\gamma_{0}} \phi\left(x_{1}, \ldots, x_{n}\right)= \begin{cases}\frac{g\left(x_{1}\right) R_{\phi}\left(x_{1}, \ldots, x_{n}\right)+\left(1-g\left(x_{1}\right)\right) \phi\left(x_{1}, \ldots, x_{n}\right)}{\left(x_{1}\right)^{d+1}}, & x_{1} \neq 0, \\ \frac{\partial_{x_{1}}^{d+1} \phi\left(0, x_{2}, \ldots, x_{n}\right)}{(d+1) !}, & x_{1}=0 .\end{cases}
$$

It is clear that $T_{\gamma_{0}} \phi$ is $C^{\infty}$ on $\left\{\left(x_{1}, \ldots, x_{n}\right): x_{1} \neq 0\right\}$ with all its partial derivatives decaying polynomially fast at $\infty$.

Moreover, since $T_{\gamma_{0}} \phi\left(x_{1}, \ldots, x_{n}\right)=R_{\phi}\left(x_{1}, \ldots, x_{n}\right) /\left(x_{1}\right)^{d+1}$ in the neighborhood $U=\left\{\left(x_{1}, \ldots, x_{n}\right):-1<x_{1}<1\right\}$ of the hyperplane $x_{1}=0$, by the Taylor remainder theorem it follows that $T_{\gamma_{0}} \phi$ is also $C^{\infty}$ in $U$ with all its partial derivatives decaying polynomially fast as $\left|\left(x_{2}, \ldots, x_{n}\right)\right| \rightarrow \infty$. Furthermore, it is not hard to see that the map $T_{\gamma_{0}}$ is continuous. Therefore, by (6.3) and (6.4)

$$
\phi\left(x_{1}, \ldots, x_{n}\right)=\left(x_{1}\right)^{d+1} T_{\gamma_{0}} \phi\left(x_{1}, \ldots, x_{n}\right)+g\left(x_{1}\right) \sum_{j=0}^{d} \frac{\partial_{x_{1}}^{j} \phi\left(0, x_{2}, \ldots, x_{n}\right)}{j !}\left(x_{1}\right)^{j} .
$$

Applying the induction hypothesis, for every $0 \leq j \leq d$ we can decompose the function $\partial_{x_{1}}^{j} \phi\left(0, x_{2}, \ldots, x_{n}\right)$ as in (6.1). Combining these decompositions into (6.5) in the obvious manner we can define the operators $T_{\gamma}$ with $|\gamma|=d+1$ and $\gamma \neq \gamma_{0}$ fulfilling (6.1). Since the trace operator $T: \mathcal{S}\left(\mathbb{R}^{n}\right) \rightarrow \mathcal{S}\left(\mathbb{R}^{n-1}\right)$ given by $T \phi\left(x_{2}, \ldots, x_{n}\right)=\phi\left(0, x_{2}, \ldots, x_{n}\right)$ is continuous and since the differential operators $\partial_{x_{1}}^{j}: \mathcal{S}\left(\mathbb{R}^{n}\right) \rightarrow \mathcal{S}\left(\mathbb{R}^{n}\right)$ are also continuous, the operators $T_{\gamma}$ are continuous as well. This completes the proof of Lemma 6.1.

Proof of Proposition 2.7. Recall that the Fourier transform $\mathcal{F}$ isomorphically maps $\mathcal{S}^{\prime}\left(\mathbb{R}^{n}\right)$ into itself. Moreover, $\mathcal{F}$ maps polynomials into linear combinations of derivatives of the point mass $\delta$ at 0 , i.e., $\mathcal{F}\left(x^{\alpha}\right)=(-i)^{-|\alpha|} \partial^{\alpha} \delta$. Therefore, Proposition 2.7 is equivalent to the following statement:

Assume that for every multi-index $\gamma$ with $|\gamma|=d+1$ the sequence $\left\{x^{\gamma} f_{i}\right\}$ converges in $\mathcal{S}^{\prime}$ as $i \rightarrow \infty$. Then there exists a sequence $\left\{a_{i, \alpha}\right\}_{i \in \mathbb{N},|\alpha| \leq d}$ such that the sequence of distributions $\left\{f_{i}-\sum_{|\alpha| \leq d} a_{i, \alpha} \partial^{\alpha} \delta\right\}$ converges in $\mathcal{S}^{\prime}$ as $i \rightarrow \infty$. 
Suppose then that $x^{\gamma} f_{i} \rightarrow f_{\gamma}$ in $\mathcal{S}^{\prime}$ as $i \rightarrow \infty$. Let $g$ be a fixed function in $\mathcal{S}\left(\mathbb{R}^{n}\right)$ such that $g(x)=1$ for all $|x| \leq 1$. For any $\varphi \in \mathcal{S}$, consider the function

$$
\phi(x)=\varphi(x)-g(x) \sum_{|\alpha| \leq d} \frac{\partial^{\alpha} \varphi(0)}{\alpha !} x^{\alpha} .
$$

Since $\phi \in \mathcal{S}_{d}\left(\mathbb{R}^{n}\right)$, it admits the decomposition (6.1). Therefore,

$$
\left\langle f_{i}, \phi\right\rangle=\left\langle f_{i}, \sum_{|\gamma|=d+1} x^{\gamma} T_{\gamma} \phi\right\rangle=\sum_{|\gamma|=d+1}\left\langle x^{\gamma} f_{i}, T_{\gamma} \phi\right\rangle \rightarrow \sum_{|\gamma|=d+1}\left\langle f_{\gamma}, T_{\gamma} \phi\right\rangle \quad \text { as } i \rightarrow \infty .
$$

On the other hand,

$$
\begin{aligned}
\left\langle f_{i}, \phi\right\rangle & =\left\langle f_{i}, \varphi\right\rangle-\sum_{|\alpha| \leq d} \frac{\partial^{\alpha} \varphi(0)}{\alpha !}\left\langle f_{i}, g(x) x^{\alpha}\right\rangle=\left\langle f_{i}, \varphi\right\rangle-\sum_{|\alpha| \leq d} \frac{\left\langle f_{i}, g(x) x^{\alpha}\right\rangle}{\alpha !}\left\langle\delta, \partial^{\alpha} \varphi\right\rangle \\
& =\left\langle f_{i}-\sum_{|\alpha| \leq d} a_{i, \alpha} \partial^{\alpha} \delta, \varphi\right\rangle \quad \text { where } a_{i, \alpha}=\frac{(-1)^{|\alpha|}\left\langle f_{i}, g(x) x^{\alpha}\right\rangle}{\alpha !} .
\end{aligned}
$$

Since $\varphi \in \mathcal{S}$ is arbitrary and the operators $T_{\gamma},|\gamma|=d+1$, are continuous by Lemma 6.1. the modified sequence $\left\{f_{i}-\sum_{|\alpha| \leq d} a_{i, \alpha} \partial^{\alpha} \delta\right\}$ converges in $\mathcal{S}^{\prime}$ as $i \rightarrow \infty$.

\subsection{Proof of Lemma 3.1.}

Proof of Lemma 3.1. Lemma 3.1 holds under a weaker hypothesis than $w \in A_{\infty}$. We only need to require that there is $N_{0}>0$ and $s_{0}>0$ such that

$$
\int_{\mathbb{R}^{n}} \frac{w^{-s_{0}}(x)}{(1+|x|)^{N_{0}}} d x<\infty .
$$

It is not hard to see that $w \in A_{\infty}$ implies (6.6). Indeed, since $w \in A_{\infty}$, we can find $1<p_{0}<\infty$ such that $w \in A_{p_{0}}$, and by duality, $w^{-p_{0}^{\prime} / p_{0}} \in A_{p_{0}^{\prime}}$, where $1 / p_{0}^{\prime}+1 / p_{0}=1$. Hence, if we let $s_{0}=p_{0}^{\prime} / p_{0}$, then $w^{-s_{0}}$ is in $A_{\infty}$ and thus it satisfies the doubling condition with respect to $\rho_{A}$. That is, there is a constant $D>0$ such that for all $x \in \mathbb{R}^{n}$ and $r>0$

$$
\int_{B_{\rho_{A}}(x,|\operatorname{det} A| r)} w^{-s_{0}}(y) d y \leq D \int_{B_{\rho_{A}}(x, r)} w^{-s_{0}}(y) d y .
$$

Choose $N_{0}>0$ such that $D|\operatorname{det} A|^{-N_{0} \zeta_{-}}<1$. Then, by (2.5) and (6.7)

$$
\begin{aligned}
& \int_{\mathbb{R}^{n}} \frac{w^{-s_{0}}(x)}{(1+|x|)^{N_{0}}} d x \leq C \int_{\mathbb{R}^{n}} \frac{w^{-s_{0}}(x)}{\left(1+\rho_{A}(x)\right)^{N_{0} \zeta_{-}}} d x \\
& =C\left[\int_{B_{\rho_{A}}(0,1)}+\sum_{j=0}^{\infty} \int_{B_{\rho_{A}}\left(0,|\operatorname{det} A|^{j+1}\right) \backslash B_{\rho_{A}}\left(0,|\operatorname{det} A|^{j}\right)}\right] \frac{w^{-s_{0}}(x)}{\left(1+\rho_{A}(x)\right)^{N_{0} \zeta_{-}}} d x \\
& \leq C \int_{B_{\rho_{A}}(0,1)} w^{-s_{0}}(x) d x \cdot\left(1+\sum_{j=0}^{\infty} \frac{D^{j+1}}{|\operatorname{det} A|^{j N_{0} \zeta_{-}}}\right)<\infty
\end{aligned}
$$

which shows (6.6). 
Suppose that $K \subset \mathbb{R}^{n}$ is compact, $0<p<\infty$ and $w$ satisfies (6.7). Let $\eta \in \mathcal{S}$ be such that $\hat{\eta}(\xi)=1$ for all $\xi \in K$. Initially, we will show that (3.5) holds for all $f \in \mathcal{S}$ with $\operatorname{supp} \hat{f} \subset K$. For any $N_{1}>0$ and $0<s_{1} \leq 1$, by $f=f * \eta$, we have

$$
\begin{aligned}
|f(x)| & \leq \int_{\mathbb{R}^{n}}|f(y)||\eta(x-y)| d y \\
& \leq \sup _{y \in \mathbb{R}^{n}} \frac{|f(y)|^{1-s_{1}}}{(1+|y|)^{N_{1}\left(1-s_{1}\right)}} \int_{\mathbb{R}^{n}}|f(y)|^{s_{1}}(1+|y|)^{N_{1}\left(1-s_{1}\right)}|\eta(x-y)| d y \\
& \leq|| f(\cdot)(1+|\cdot|)^{-N_{1}} \|_{\infty}^{1-s_{1}} \sup _{y \in \mathbb{R}^{n}}|\eta(x-y)|(1+|y|)^{N_{1}} \int_{\mathbb{R}^{n}} \frac{|f(y)|^{s_{1}}}{(1+|y|)^{N_{1} s_{1}}} d y \\
& \leq C(1+|x|)^{N_{1}}|| f(\cdot)(1+|\cdot|)^{-N_{1}} \|_{\infty}^{1-s_{1}} \int_{\mathbb{R}^{n}} \frac{|f(y)|^{s_{1}}}{(1+|y|)^{N_{1} s_{1}}} d y,
\end{aligned}
$$

since $\eta$ is in $\mathcal{S}$. Therefore,

$$
\sup _{x \in \mathbb{R}^{n}} \frac{|f(x)|}{(1+|x|)^{N_{1}}} \leq C\left(\int_{\mathbb{R}^{n}} \frac{|f(y)|^{s_{1}}}{(1+|y|)^{N_{1} s_{1}}} d y\right)^{1 / s_{1}} .
$$

If $0<s_{1}<p$, then by Hölder's inequality,

$$
\begin{aligned}
\int \frac{|f(y)|^{s_{1}}}{(1+|y|)^{N_{1} s_{1}}} & \frac{w^{s_{1} / p}(y)}{w^{s_{1} / p}(y)} d y \\
& \leq\left(\int|f(y)|^{p} w(y) d y\right)^{\frac{s_{1}}{p}}\left(\int \frac{w^{-s_{1} /\left(p-s_{1}\right)}(y)}{(1+|y|)^{N_{1} p s_{1} /\left(p-s_{1}\right)}} d y\right)^{\frac{p-s_{1}}{p}} .
\end{aligned}
$$

Therefore, if we choose $0<s_{1}<p$ small enough so that $s_{1} /\left(p-s_{1}\right)<s_{0}$ and $N_{1}>0$ large enough so that $N_{1} p s_{1} /\left(p-s_{1}\right)>N_{0}$, where $N_{0}$ and $s_{0}$ are the same as in (6.6), then combining (6.6), (6.8), and (6.9) yields (3.5) with $N=N_{1}$.

Finally, to remove the assumption that $f \in \mathcal{S}$, we use the standard regularization technique as in Lemma 3.4. Let $h \in \mathcal{S}$ satisfy $\operatorname{supp} \hat{h} \subset B(0,1), \hat{h}(\xi) \geq 0$, and $h(0)=1$. By the Fourier Inversion Formula, $|h(x)| \leq 1$ for all $x \in \mathbb{R}^{n}$. Given an arbitrary $f \in \mathcal{S}^{\prime}$ with supp $\hat{f} \subset K$, we let $f_{\delta}(x)=f(x) h(\delta x)$ for $0<\delta<1$. Then $\operatorname{supp} \hat{f}_{\delta} \subset K+B(0,1), f_{\delta} \in \mathcal{S},\left|f_{\delta}(x)\right| \leq|f(x)|$ for all $x$, and $f_{\delta}(x) \rightarrow f(x)$ uniformly on compact sets as $\delta \rightarrow 0$. Applying (3.5) to $f_{\delta}$ and letting $\delta \rightarrow 0$, we obtain (3.5) for a general $f \in \mathcal{S}^{\prime}$ with supp $\hat{f} \subset K$. This completes the proof of Lemma 3.1

Finally, we remark that an alternative proof of Lemma 3.1, which also works for doubling measures with respect to $\rho_{A}$ (hence, a larger class than $A_{\infty}$ weights), can be found in [7].

6.3. Proof of Lemma 3.3. We need an auxiliary lemma to show Lemma 3.3 .

Lemma 6.2. Suppose $0<a \leq r<\infty, \lambda>r / a$, and $i, j \in \mathbb{Z}$. Then for any sequence $s=\left\{s_{P}\right\}_{P}$ and for each cube $Q \in \mathcal{Q}$ with $|Q|=|\operatorname{det} A|^{-j}$ and each 
$x \in Q$, we have

$$
\begin{aligned}
& \left(\sum_{|P|=|\operatorname{det} A|^{-i}}\left|s_{P}\right|^{r} /\left(1+\frac{\rho_{A}\left(x_{Q}-x_{P}\right)}{\max (|P|,|Q|)}\right)^{\lambda}\right)^{1 / r} \\
& \quad \leq C|\operatorname{det} A|^{(i-j)_{+} / a}\left(M_{\rho_{A}}\left(\sum_{|P|=|\operatorname{det} A|^{-i}}\left|s_{P}\right|^{a} \chi_{P}\right)(x)\right)^{1 / a},
\end{aligned}
$$

where the constant $C$ depends only on $\lambda-r / a$. In particular, if $i=j$, then

$$
\sum_{|Q|=|\operatorname{det} A|^{-j}}\left(s_{r, \lambda}^{*}\right)_{Q} \tilde{\chi}_{Q} \leq C\left(M_{\rho_{A}}\left(\sum_{|Q|=|\operatorname{det} A|^{-j}}\left|s_{Q}\right| \tilde{\chi}_{Q}\right)^{a}\right)^{1 / a}
$$

with the same constant $C$.

Proof. We may assume $x_{Q}=0$. Consider first the case when $i \leq j$. For $k \geq 1$, let

$$
\begin{aligned}
& A_{0}=\left\{P \in \mathcal{Q}:|P|=|\operatorname{det} A|^{-i} \quad \text { and } \quad \rho_{A}\left(x_{P}\right) /|P| \leq 1\right\}, \\
& A_{k}=\left\{P \in \mathcal{Q}:|P|=|\operatorname{det} A|^{-i} \quad \text { and } \quad|\operatorname{det} A|^{k-1}<\rho_{A}\left(x_{P}\right) /|P| \leq|\operatorname{det} A|^{k}\right\} .
\end{aligned}
$$

Then

$$
\begin{aligned}
& \sum_{P \in A_{k}} \frac{\left|s_{P}\right|^{r}}{\left(1+\rho_{A}\left(x_{P}\right) /|P|\right)^{\lambda}} \\
& \quad \leq C|\operatorname{det} A|^{-k \lambda} \sum_{P \in A_{k}}\left|s_{P}\right|^{r} \leq C|\operatorname{det} A|^{-k \lambda}\left(\sum_{P \in A_{k}}\left|s_{P}\right|^{a}\right)^{r / a} \\
& \quad \leq C|\operatorname{det} A|^{-k \lambda}|\operatorname{det} A|^{r i / a}\left(\int_{\tilde{B}} \sum_{P \in A_{k}}\left|s_{P}\right|^{a} \chi_{P}\right)^{r / a},
\end{aligned}
$$

since $\left(\int \chi_{P}\right)^{r / a}=|\operatorname{det} A|^{-r i / a}$ and $\bigcup_{P \in A_{k}} P \subset \tilde{B}:=B_{\rho_{A}}\left(0,2 H|\operatorname{det} A|^{k-i}\right)$. Hence, by the definition of the maximal operator, we have

$$
\begin{aligned}
& \sum_{P \in A_{k}} \frac{\left|s_{P}\right|^{r}}{\left(1+\rho_{A}\left(x_{P}\right) /|P|\right)^{\lambda}} \\
& \quad \leq C|\operatorname{det} A|^{-k \lambda}|\operatorname{det} A|^{r i / a}|\tilde{B}|^{r / a}\left(\frac{1}{|\tilde{B}|} \int_{\tilde{B}} \sum_{P \in A_{k}}\left|s_{P}\right|^{a} \chi_{P}\right)^{r / a} \\
& \quad \leq C|\operatorname{det} A|^{-k(\lambda-r / a)}\left(M_{\rho_{A}}\left(\sum_{|P|=|\operatorname{det} A|^{-i}}\left|s_{P}\right|^{a} \chi_{P}\right)(x)\right)^{r / a}
\end{aligned}
$$

for any $x \in Q \subset \tilde{B}$. Summing over $k \geq 0$, yields (6.10).

In the second case $i>j$, we redefine for $k \geq 1$,

$$
\begin{aligned}
& A_{0}=\left\{P \in \mathcal{Q}:|P|=|\operatorname{det} A|^{-i} \quad \text { and } \quad \rho_{A}\left(x_{P}\right) /|Q| \leq 1\right\}, \\
& A_{k}=\left\{P \in \mathcal{Q}:|P|=|\operatorname{det} A|^{-i} \quad \text { and } \quad|\operatorname{det} A|^{k-1}<\rho_{A}\left(x_{P}\right) /|Q| \leq|\operatorname{det} A|^{k}\right\} .
\end{aligned}
$$


Then as before

$$
\begin{gathered}
\sum_{P \in A_{k}} \frac{\left|s_{P}\right|^{r}}{\left(1+\rho_{A}\left(x_{P}\right) /|Q|\right)^{\lambda}} \leq C|\operatorname{det} A|^{-k \lambda}|\operatorname{det} A|^{r i / a}\left(\int_{\tilde{B}} \sum_{P \in A_{k}}\left|s_{P}\right|^{a} \chi_{P}\right)^{r / a} \\
\leq C|\operatorname{det} A|^{(i-j) r / a-k(\lambda-r / a)}\left(M_{\rho_{A}}\left(\sum_{|P|=|\operatorname{det} A|^{-i}}\left|s_{P}\right|^{a} \chi_{P}\right)(x)\right)^{r / a}
\end{gathered}
$$

for any $x \in Q \subset \tilde{B}:=B_{\rho_{A}}\left(0,2 H|\operatorname{det} A|^{k-j}\right)$. Summing over $k \geq 0$, yields (6.10).

To see (6.11), multiply both sides of (6.10) by $\tilde{\chi}_{Q}$, and sum over all $Q \in \mathcal{Q}$ with $|Q|=|\operatorname{det} A|^{-j}$,

$$
\begin{array}{r}
\sum_{|Q|=|\operatorname{det} A|^{-j}}\left(s_{r, \lambda}^{*}\right)_{Q} \tilde{\chi}_{Q} \leq C \sum_{|Q|=|\operatorname{det} A|^{-j}}\left(M_{\rho_{A}}\left(\sum_{|P|=|\operatorname{det} A|^{-j}}\left|s_{P}\right| \tilde{\chi}_{P}\right)^{a}\right)^{1 / a} \chi_{Q} \\
=C\left(M_{\rho_{A}}\left(\sum_{|P|=|\operatorname{det} A|^{-j}}\left|s_{P}\right| \tilde{\chi}_{P}\right)^{a}\right)^{1 / a},
\end{array}
$$

since $\left\{Q \in \mathcal{Q}:|Q|=|\operatorname{det} A|^{-j}\right\}$ is a partition of $\mathbb{R}^{n}$.

Proof of Lemma 3.3. Suppose $r>0$ and $\lambda>\max \left(1, r / q, r p_{0} / p\right)$. If $r<\min \left(q, p / p_{0}\right)$, then set $a=r$. Otherwise, if $r \geq \min \left(q, p / p_{0}\right)$, then take $a$ such that $r / \lambda<a<$ $\min \left(r, q, p / p_{0}\right)$. It is possible to choose such an $a$, since $\lambda>\max \left(1, r / q, r p_{0} / p\right)$ implies $r / \lambda<\min \left(r, q, p / p_{0}\right)$. In both cases we have that

$$
0<a \leq r<\infty, \quad \lambda>r / a, \quad q / a>1, \quad p / a>p_{0} .
$$

Therefore, Lemma 6.2 yields (6.11), and consequently we can estimate $\dot{\mathbf{f}}_{p}^{\alpha, q}(A, w d x)$ quasi-norm of $s_{r, \lambda}^{*}$ by

$$
\left\|s_{r, \lambda}^{*}\right\|_{\dot{\mathbf{f}}_{p}^{\alpha, q}(A, w d x)} \leq C\left\|\left(\sum_{j \in \mathbb{Z}}\left(M_{\rho_{A}}\left(\sum_{|Q|=|\operatorname{det} A|^{-j}}|Q|^{-\alpha}\left|s_{Q}\right| \tilde{\chi}_{Q}\right)^{a}\right)^{q / a}\right)^{a / q}\right\|_{L^{p / a}(w)}^{1 / a} .
$$

Since $q / a>1$ and $p / a>p_{0}$, by the Stein-Fefferman vector-valued maximal inequality we can remove $M_{\rho_{A}}$ from the above estimate (by increasing a constant $C$ ) to obtain

$$
\left\|s_{r, \lambda}^{*}\right\|_{\dot{\mathbf{f}}_{p}^{\alpha, q}(A, w d x)} \leq C\|s\|_{\dot{\mathbf{f}}_{p}^{\alpha, q}(A, w d x)} .
$$

\subsection{Proof of Lemma 3.4.}

Proof of Lemma 3.4. Since $g \in \mathcal{S}^{\prime}\left(\mathbb{R}^{n}\right)$ has compact support in the Fourier domain, $g$ is regular. More precisely, the distribution $g$ is a slowly increasing (at most polynomially fast) and infinitely differentiable function. Let $\gamma \in \mathcal{S}\left(\mathbb{R}^{n}\right)$ be such that $\hat{\gamma}(\xi)=1$ for all $\xi \in K$. By $g=\gamma * g$, we have for $i=1,2, \ldots, n$,

$$
\begin{aligned}
& \left|\partial_{i} g(x-y)\right|=\left|\left(\partial_{i} \gamma * g\right)(x-y)\right| \leq \int_{\mathbb{R}^{n}}\left|\partial_{i} \gamma(z-y)\right||g(x-z)| d z \\
& \quad \leq C \int_{\mathbb{R}^{n}}\left|\partial_{i} \gamma(z-y)\right|\left(1+\rho_{A}(z-y)\right)^{1 / r}\left(1+\rho_{A}(y)\right)^{1 / r} \frac{|g(x-z)|}{\left(1+\rho_{A}(z)\right)^{1 / r}} d z,
\end{aligned}
$$

since

$$
1+\rho_{A}(z) \leq H\left(1+\rho_{A}(z-y)+\rho_{A}(y)\right) \leq H\left(1+\rho_{A}(z-y)\right)\left(1+\rho_{A}(y)\right) .
$$


Hence,

$$
\begin{aligned}
& \left|\partial_{i} g(x-y)\right| \\
& \quad \leq H \sup _{z \in \mathbb{R}^{n}} \frac{|g(x-z)|}{\left(1+\rho_{A}(z)\right)^{1 / r}}\left(1+\rho_{A}(y)\right)^{1 / r} \int_{\mathbb{R}^{n}}\left|\partial_{i} \gamma(z-y)\right|\left(1+\rho_{A}(z-y)\right)^{1 / r} d z .
\end{aligned}
$$

Since $\partial_{i} \gamma \in \mathcal{S}$, there exists $C_{1}>0$ such that

$$
\sup _{y \in \mathbb{R}^{n}} \frac{|\nabla g(x-y)|}{\left(1+\rho_{A}(y)\right)^{1 / r}} \leq C_{1} \sup _{z \in \mathbb{R}^{n}} \frac{|g(x-z)|}{\left(1+\rho_{A}(z)\right)^{1 / r}} \quad \text { for all } x \in \mathbb{R}^{n} .
$$

To show the second inequality in (3.8), take $\delta$ such that $0<\delta<1$. By the Mean-Value Theorem we have

$$
\begin{aligned}
|g(x-y)| \leq & \min _{z \in B_{\rho_{A}}(x-y, \delta)}|g(z)|+\operatorname{diam}\left(B_{\rho_{A}}(x-y, \delta)\right) \sup _{\tilde{z} \in B_{\rho_{A}}(x-y, \delta)}|\nabla g(\tilde{z})|, \\
& \leq C \delta^{-1 / r}\left(\int_{B_{\rho_{A}}(x-y, \delta)}|g(z)|^{r} d z\right)^{1 / r}+C \delta^{\zeta-} \sup _{\tilde{z} \in B_{\rho_{A}}(x-y, \delta)}|\nabla g(\tilde{z})|
\end{aligned}
$$

by Lemma2.2. where $\zeta_{-}$is the same as in (2.6). Here, we also used that $B_{\rho_{A}}(x-y, \delta)$ is convex, since by remarks preceding Proposition 2.1] it is possible to find a quasinorm $\rho_{A}$, which produces convex $\rho_{A}$-balls. Since

$$
B_{\rho_{A}}(x-y, \delta) \subset B_{\rho_{A}}\left(x, H\left(\delta+\rho_{A}(y)\right)\right),
$$

we have

$$
\begin{gathered}
\int_{B_{\rho_{A}}(x-y, \delta)}|g(z)|^{r} d z \leq \int_{B_{\rho_{A}}\left(x, H\left(\delta+\rho_{A}(y)\right)\right)}|g(z)|^{r} d z \leq C\left(\delta+\rho_{A}(y)\right) M_{\rho_{A}}\left(|g|^{r}\right)(x), \\
\sup _{\tilde{z} \in \mathbb{B}_{\rho_{A}}(x-y, \delta)}|\nabla g(\tilde{z})| \leq \sup _{z \in B_{\rho_{A}}\left(x, H\left(\delta+\rho_{A}(y)\right)\right)}|\nabla g(x-z)| \\
\quad \leq H^{1 / r}\left(1+\delta+\rho_{A}(y)\right)^{1 / r} \sup _{z \in \mathbb{R}^{n}} \frac{|\nabla g(x-z)|}{\left(1+\rho_{A}(z)\right)^{1 / r}} .
\end{gathered}
$$

Combining the above estimates we have

$$
|g(x-y)| \leq C\left(1+\rho_{A}(y)\right)^{1 / r}\left[\delta^{-1 / r}\left(M_{\rho_{A}}\left(|g|^{r}\right)(x)\right)^{1 / r}+\delta^{\zeta-} \sup _{z \in \mathbb{R}^{n}} \frac{|\nabla g(x-z)|}{\left(1+\rho_{A}(z)\right)^{1 / r}}\right] .
$$

Therefore,

$$
\sup _{z \in \mathbb{R}^{n}} \frac{|g(x-z)|}{\left(1+\rho_{A}(z)\right)^{1 / r}} \leq C\left[\delta^{-1 / r}\left(M_{\rho_{A}}\left(|g|^{r}\right)(x)\right)^{1 / r}+\delta^{\zeta_{-}} \sup _{z \in \mathbb{R}^{n}} \frac{|\nabla g(x-z)|}{\left(1+\rho_{A}(z)\right)^{1 / r}}\right] .
$$

Assume for the moment that $g \in \mathcal{S}$, and choose $\delta$ sufficiently small in order to have $C_{1} C \delta^{\zeta_{-}}<1 / 2$. Combining the above with (6.12) and $\sup _{z \in \mathbb{R}^{n}} \frac{|g(x-z)|}{\left(1+\rho_{A}(z)\right)^{1 / r}}<\infty$, we obtain

$$
\sup _{z \in \mathbb{R}^{n}} \frac{|g(x-z)|}{\left(1+\rho_{A}(z)\right)^{1 / r}} \leq C_{2}\left(M_{\rho_{A}}\left(|g|^{r}\right)(x)\right)^{1 / r} \quad \text { for all } x \in \mathbb{R}^{n} .
$$

To remove the assumption that $g \in \mathcal{S}$, we apply the standard regularization argument; see, e.g. [42, p. 22] or [27, Lemma A.4]. Let $h \in \mathcal{S}$ satisfy supp $\hat{h} \subset$ $B(0,1), \hat{h}(\xi) \geq 0$, and $h(0)=1$. By the Fourier Inversion Formula, $|h(x)| \leq 1$ for all $x \in \mathbb{R}^{n}$. For $0<\delta<1$, let $g_{\delta}(x)=g(x) h(\delta x)$. Then supp $\hat{g}_{\delta} \subset K+B(0,1)$, $g_{\delta} \in \mathcal{S},\left|g_{\delta}(x)\right| \leq|g(x)|$ for all $x$, and $g_{\delta}(x) \rightarrow g(x)$ uniformly on compact sets as 
$\delta \rightarrow 0$. Applying (6.13) to $g_{\delta}$ and letting $\delta \rightarrow 0$, we obtain (6.13) for a general $g \in \mathcal{S}^{\prime}$. This completes the proof of Lemma 3.4.

6.5. Proof of Lemma 5.1. To prove Lemma 5.1 we need to show Lemma 6.3, which is a generalization of the corresponding dyadic result; see [27, Lemma B.1].

Lemma 6.3. Suppose $A$ is an expansive matrix, $L \in \mathbb{Z}, L \geq 0, R>1, S>1+L \zeta_{+}$, $i, j \in \mathbb{Z}, i \geq j$, and $x_{0} \in \mathbb{R}^{n}$. Suppose that $g \in C^{L+1}\left(\mathbb{R}^{n}\right)$ and $h \in L^{1}\left(\mathbb{R}^{n}\right)$ satisfy

$$
\begin{gathered}
\left|\partial^{\gamma}\left[g\left(A^{-j} \cdot\right)\right](x)\right| \leq|\operatorname{det} A|^{j / 2}\left(1+\rho_{A}(x)\right)^{-R} \quad \text { for }|\gamma| \leq L+1, \\
|h(x)| \leq|\operatorname{det} A|^{i / 2}\left(1+\rho_{A}\left(A^{i}\left(x-x_{0}\right)\right)\right)^{-\max (R, S)}, \\
\int x^{\gamma} h(x) d x=0 \quad \text { for }|\gamma| \leq L .
\end{gathered}
$$

Then for any $0<\theta \leq 1$ satisfying

$$
(L+\theta) \zeta_{-}+1<S,
$$

there exists a constant $C>0$, which is independent of $g, h, i, j$, and $x_{0}$, such that $(6.18)$

$|(g * h)(x)| \leq C|\operatorname{det} A|^{-(i-j)\left((L+\theta) \zeta_{-}+1 / 2\right)}\left(1+\rho_{A}\left(A^{j}\left(x-x_{0}\right)\right)\right)^{-R} \quad$ for all $x \in \mathbb{R}^{n}$.

Proof. Using translation and dilation, we may assume that $j=0$ and $x_{0}=0$. Indeed, suppose that $g$ and $h$ satisfy (6.14) for some $i_{0} \geq j_{0} \in \mathbb{Z}$ and $\tilde{x}_{0} \in \mathbb{R}^{n}$. Then $|\operatorname{det} A|^{-j_{0} / 2} D_{A^{-j_{0}}} g(x)$ and $|\operatorname{det} A|^{-j_{0} / 2} D_{A^{-j_{0}}} T_{-\tilde{x}_{0}} h(x)$ satisfy the corresponding conditions for $i=i_{0}-j_{0}, j=0$, and $x_{0}=0$. Thus, assuming Lemma 6.3 holds for $j=0$ and $x_{0}$,

$|\operatorname{det} A|^{-j_{0}}\left|\left(D_{A^{-j_{0}}} g * D_{A^{-j_{0}}} T_{-\tilde{x}_{0}} h\right)(x)\right| \leq C|\operatorname{det} A|^{-\left(i_{0}-j_{0}\right)\left((L+\theta) \zeta_{-}+1 / 2\right)}\left(1+\rho_{A}(x)\right)^{-R}$.

This shows the general case of Lemma 6.3, since

$|\operatorname{det} A|^{-j_{0}}\left(D_{A^{-j_{0}}} g * D_{A^{-j_{0}}} T_{-\tilde{x}_{0}} h\right)(x)=\left(g * T_{-\tilde{x}_{0}} h\right)\left(A^{-j_{0}} x\right)=(g * h)\left(A^{-j_{0}} x+\tilde{x}_{0}\right)$.

Given $x \in \mathbb{R}^{n}$ we decompose $\mathbb{R}^{n}$ into 3 domains,

$$
\begin{aligned}
& D_{1}=\left\{y \in \mathbb{R}^{n}: \rho_{A}(y-x)<1\right\}, \\
& D_{2}=\left\{y \in \mathbb{R}^{n}: \rho_{A}(y-x) \geq 1 \quad \text { and } \quad \rho_{A}(y) \leq \rho_{A}(x) / 2 H\right\}, \\
& D_{3}=\left\{y \in \mathbb{R}^{n}: \rho_{A}(y-x) \geq 1 \quad \text { and } \quad \rho_{A}(y)>\rho_{A}(x) / 2 H\right\},
\end{aligned}
$$

where $H$ is the constant of the quasi-subadditivity inequality.

Then by the vanishing moment condition (6.16) we have

$$
|(g * h)(x)| \leq \int_{\mathbb{R}^{n}}\left|g(y)-\sum_{|\beta| \leq L} \frac{\partial^{\beta} g(x)}{\beta !}(y-x)^{\beta}\right||h(x-y)| d y \equiv \int_{D_{1}}+\int_{D_{2}}+\int_{D_{3}} .
$$

Case 1: Estimation of $D_{1}$. For $y \in D_{1}$, applying the Taylor Remainder Theorem and (6.14) with $|\gamma|=L+1$, we have

$$
\begin{aligned}
& \left|g(y)-\sum_{|\beta| \leq L} \frac{\partial^{\beta} g(x)}{\beta !}(y-x)^{\beta}\right| \leq C|x-y|^{L+1} \sup _{z \in[x, y]|\beta|=L+1} \sup _{|\beta|=L}\left|\partial^{\beta} g(z)\right| \\
& \leq C|x-y|^{L+1} \sup _{z \in[x, y]}\left(1+\rho_{A}(z)\right)^{-R} \leq C \rho_{A}(x-y)^{(L+1) \zeta_{-}}\left(1+\rho_{A}(x)\right)^{-R},
\end{aligned}
$$


where $[x, y]$ is the line segment joining $x$ and $y$. Here, we used that for $y \in D_{1}$ and $z \in[x, y]$,

$$
\rho_{A}(z) \geq(1 / H) \rho_{A}(x)-\rho_{A}(x-z) \geq(1 / H) \rho_{A}(x)-\rho_{A}(x-y) \geq(1 / H) \rho_{A}(x)-1,
$$

where $\rho_{A}(x-y) \geq \rho_{A}(x-z)$ for $z \in[x, y]$ is a consequence of convexity of $\rho_{A^{-}}$ balls. Suppose that $0<\theta \leq 1$ satisfies (6.17). Such $\theta$ can always be chosen, since $L \zeta_{-}+1 \leq L \zeta_{+}+1<S$. Hence, by the change of variables we have

$$
\begin{aligned}
\int_{D_{1}} \leq & C|\operatorname{det} A|^{i / 2}\left(1+\rho_{A}(x)\right)^{-R} \int_{D_{1}} \rho_{A}(x-y)^{(L+\theta) \zeta_{-}}\left(1+\rho_{A}\left(A^{i}(x-y)\right)\right)^{-S} d y \\
\leq & C|\operatorname{det} A|^{i / 2}\left(1+\rho_{A}(x)\right)^{-R} \int_{\mathbb{R}^{n}} \rho_{A}(y)^{(L+\theta) \zeta_{-}}\left(1+\rho_{A}\left(A^{i} y\right)\right)^{-S} d y \\
\leq & C|\operatorname{det} A|^{i / 2}\left(1+\rho_{A}(x)\right)^{-R}|\operatorname{det} A|^{-i\left((L+\theta) \zeta_{-}+1\right)} \\
& \times \int_{\mathbb{R}^{n}} \rho_{A}(y)^{(L+\theta) \zeta_{-}}\left(1+\rho_{A}(y)\right)^{-S} d y \\
\leq & C|\operatorname{det} A|^{-i\left((L+\theta) \zeta_{-}+1 / 2\right)}\left(1+\rho_{A}(x)\right)^{-R}
\end{aligned}
$$

since the last integral is finite by (6.17).

Case 2: Estimation of $D_{2}$. For $y \in D_{2}$, we have

$$
\rho_{A}(x-y) \geq \rho_{A}(x) / H-\rho_{A}(y) \geq \rho_{A}(x) / H-\rho_{A}(x) /(2 H)=\rho_{A}(x) /(2 H) .
$$

On the other hand,

$$
\rho_{A}(x-y) \leq H\left(\rho_{A}(x)+\rho_{A}(y)\right) \leq H \rho_{A}(x)+\rho_{A}(x) / 2=(H+1 / 2) \rho_{A}(x) .
$$

Thus, we have

$$
1+\rho_{A}\left(A^{i}(x-y)\right) \geq|\operatorname{det} A|^{i} \rho_{A}(x-y) \geq|\operatorname{det} A|^{i}\left(1+\rho_{A}(x)\right) /(4 H)
$$

and

$$
|x-y|^{L} \leq C \rho_{A}(x-y)^{L \zeta_{+}} \leq C \rho_{A}(x)^{L \zeta_{+}} .
$$

Consequently,

$$
\begin{aligned}
\int_{D_{2}} \leq & C \int_{D_{2}}\left[\left(1+\rho_{A}(y)\right)^{-R}+\sum_{|\beta| \leq L} \frac{|x-y|^{|\beta|}}{\left(1+\rho_{A}(x)\right)^{R}}\right] \frac{|\operatorname{det} A|^{i / 2}}{\left(1+\rho_{A}\left(A^{i}(x-y)\right)\right)^{\max (R, S)}} d y \\
\leq & C \frac{|\operatorname{det} A|^{-i(S-1 / 2)}}{\left(1+\rho_{A}(x)\right)^{\max (R, S)}} \\
& \times\left[\int_{\mathbb{R}^{n}} \frac{1}{\left(1+\rho_{A}(y)\right)^{R}} d y+\frac{\rho_{A}(x)^{L \zeta_{+}}}{\left(1+\rho_{A}(x)\right)^{R}} \int_{\rho_{A}(y) \leq \rho_{A}(x) / 2 H} d y\right] \\
\leq & C \frac{|\operatorname{det} A|^{-i(S-1 / 2)}}{\left(1+\rho_{A}(x)\right)^{R}}\left[1+\frac{\rho_{A}(x)^{L \zeta_{+}+1}}{\left(1+\rho_{A}(x)\right)^{S}}\right] \\
\leq & C|\operatorname{det} A|^{-i(S-1 / 2)}\left(1+\rho_{A}(x)\right)^{-R},
\end{aligned}
$$

since $S>L \zeta_{+}+1$. This is exactly what is needed, since $S-1 / 2>(L+\theta) \zeta_{-}+1 / 2$ by (6.17). 
Case 3: Estimation of $D_{3}$. For $y \in D_{3}$, we have $\rho_{A}(y) \geq \rho_{A}(x) / 2 H$ and, hence,

$$
\begin{aligned}
\int_{D_{3}} & \leq C \int_{D_{3}}\left[\left(1+\rho_{A}(y)\right)^{-R}+\sum_{|\beta| \leq L} \frac{|x-y|^{|\beta|}}{\left(1+\rho_{A}(x)\right)^{R}}\right] \frac{|\operatorname{det} A|^{i / 2}}{\left(1+\rho_{A}\left(A^{i}(x-y)\right)\right)^{\max (R, S)}} d y \\
& \leq C \frac{|\operatorname{det} A|^{i / 2}}{\left(1+\rho_{A}(x)\right)^{R}} \int_{\rho_{A}(x-y) \geq 1} \frac{\rho_{A}(x-y)^{L \zeta_{+}}}{\rho_{A}\left(A^{i}(x-y)\right)^{S}} d y \\
& \leq C \frac{|\operatorname{det} A|^{-i(S-1 / 2)}}{\left(1+\rho_{A}(x)\right)^{R}} \int_{\rho_{A}(z) \geq 1} \rho_{A}(z)^{L \zeta_{+}-S} d z \leq C \frac{|\operatorname{det} A|^{-i(S-1 / 2)}}{\left(1+\rho_{A}(x)\right)^{R}},
\end{aligned}
$$

since $S>L \zeta_{+}+1$. Again, this is what is needed by (6.17).

Combining the above estimates yields (6.18) and completes the proof of Lemma 6.3 .

As a special case of Lemma 6.3 formally corresponding to $L=-1$, where no vanishing moments on $h$ are assumed, we obtain

Lemma 6.4. Suppose $A$ is an expansive matrix, $R>1, i, j \in \mathbb{Z}, i \geq j$, and $x_{0} \in \mathbb{R}^{n}$. Suppose $g, h \in L^{1}\left(\mathbb{R}^{n}\right)$ satisfy

$$
|g(x)| \leq|\operatorname{det} A|^{j / 2}\left(1+\rho_{A}\left(A^{j} x\right)\right)^{-R}
$$

and

$$
|h(x)| \leq|\operatorname{det} A|^{i / 2}\left(1+\rho_{A}\left(A^{i}\left(x-x_{0}\right)\right)\right)^{-R} .
$$

Then

$$
|(g * h)(x)| \leq C|\operatorname{det} A|^{-(i-j) / 2}\left(1+\rho_{A}\left(A^{j}\left(x-x_{0}\right)\right)\right)^{-R}
$$

for some constant $C>0$.

Finally, we are ready to show Lemma 5.1

Proof of Lemma 5.1. We will split our estimates into 4 cases.

Case 1. Suppose $|Q| \leq|P|$ and $\alpha \geq 0$. Let $i, j \in \mathbb{Z}$ be such that $|Q|=$ $|\operatorname{det} A|^{-i} \leq|\operatorname{det} A|^{-j}=|P|$. Then it is not hard to check that $g(x)=\Psi_{P}\left(x_{P}-x\right)$ and $h(x)=\overline{\Phi_{Q}(x)}$ satisfy the hypotheses of Lemma 6.3 with $R=M, L=\left\lfloor\alpha / \zeta_{-}\right\rfloor$, $S=1+\alpha \zeta_{+} / \zeta_{-}+M-J$ and $x_{0}=x_{Q}$. More precisely, (5.1), (5.5), and (5.6) imply (6.14), (6.15), and (6.16), respectively. Indeed, to show the least obvious implication (5.1) $\Longrightarrow$ (6.14) it suffices to observe that

$$
\begin{aligned}
\partial^{\gamma}\left(D_{A^{-j}} g\right)(x) & =\partial^{\gamma}\left(D_{A^{-j}} T_{x_{P}} D_{-I d} \Psi_{P}\right)(x)=\partial^{\gamma}\left(T_{A^{j} x_{P}} D_{-I d} D_{A^{-j}} \Psi_{P}\right)(x) \\
& =\partial^{\gamma}\left(D_{-I d} D_{A^{-j}} \Psi_{P}\right)\left(x-A^{j} x_{P}\right)=(-1)^{|\gamma|} \partial^{\gamma}\left(D_{A^{-j}} \Psi_{P}\right)\left(A^{j} x_{P}-x\right) .
\end{aligned}
$$

Therefore, by Lemma 6.3 with $\theta=\min \left(1, \alpha / \zeta_{-}\left\lfloor\alpha / \zeta_{-}\right\rfloor+(M-J) /\left(2 \zeta_{-}\right)\right)$we have

$$
\begin{aligned}
\left|\left\langle\Psi_{P}, \Phi_{Q}\right\rangle\right| & =\left|(g * h)\left(x_{P}\right)\right| \\
& \leq C|\operatorname{det} A|^{-(i-j)\left((L+\theta) \zeta_{-}+1 / 2\right)}\left(1+|\operatorname{det} A|^{j} \rho_{A}\left(x_{Q}-x_{P}\right)\right)^{-M} \\
& \leq C|\operatorname{det} A|^{-(i-j)(\alpha+\epsilon / 2+1 / 2)}\left(1+|\operatorname{det} A|^{j} \rho_{A}\left(x_{Q}-x_{P}\right)\right)^{-J-\epsilon},
\end{aligned}
$$

where $\epsilon / 2=(L+\theta) \zeta_{-}-\alpha>0$. 
Case 2. Suppose $|Q| \leq|P|$ and $\alpha<0$. Let $i, j \in \mathbb{Z}$ be such that $|Q|=$ $|\operatorname{det} A|^{-i} \leq|\operatorname{det} A|^{-j}=|P|$. By Lemma 6.4 with $R=M$ for the same choice of $g$ and $h$ as in Case 1 , we have

$$
\begin{aligned}
\left|\left\langle\Psi_{P}, \Phi_{Q}\right\rangle\right| & \leq C|\operatorname{det} A|^{-(i-j) / 2}\left(1+|\operatorname{det} A|^{j} \rho_{A}\left(x_{Q}-x_{P}\right)\right)^{-M} \\
& \leq C|\operatorname{det} A|^{-(i-j)(\alpha+\epsilon / 2+1 / 2)}\left(1+|\operatorname{det} A|^{j} \rho_{A}\left(x_{Q}-x_{P}\right)\right)^{-J-\epsilon},
\end{aligned}
$$

where $\epsilon / 2=\min (-\alpha,(M-J) / 2)>0$.

Case 3. Suppose $|Q|>|P|$ and $N \geq 0$. Let $i, j \in \mathbb{Z}$ be such that $|Q|=$ $|\operatorname{det} A|^{-j}>|\operatorname{det} A|^{-i}=|P|$. Again, it is not hard to check that hypotheses of Lemma 6.3 with $R=M, L=N, S=(M-\alpha) \zeta_{+} / \zeta_{-}, x_{0}=x_{P}, g(x)=\overline{\Phi_{Q}\left(x_{Q}-x\right)}$, and $h(x)=\Psi_{P}(x)$ are satisfied. More precisely, (5.2), (5.3), and (5.4) imply (6.15), (6.16), and (6.14), respectively. Therefore, by Lemma 6.3 with

$$
\theta=\min \left(1,(J-\alpha-1) / \zeta_{-}-\left\lfloor(J-\alpha-1) / \zeta_{-}\right\rfloor+(M-J) /\left(2 \zeta_{-}\right)\right)
$$

we have

$$
\begin{aligned}
\left|\left\langle\Psi_{P}, \Phi_{Q}\right\rangle\right| & =\left|(g * h)\left(x_{Q}\right)\right| \\
& \leq C|\operatorname{det} A|^{-(i-j)\left((N+\theta) \zeta_{-}+1 / 2\right)}\left(1+|\operatorname{det} A|^{j} \rho_{A}\left(x_{Q}-x_{P}\right)\right)^{-M} \\
& \leq C|\operatorname{det} A|^{-(i-j)(J-\alpha+\epsilon / 2-1 / 2)}\left(1+|\operatorname{det} A|^{j} \rho_{A}\left(x_{Q}-x_{P}\right)\right)^{-J-\epsilon},
\end{aligned}
$$

where $\epsilon / 2=(N+\theta) \zeta_{-}-(J-\alpha-1)>0$.

Case 4. Finally, suppose $|Q|>|P|$ and $N=-1$. Let $i, j \in \mathbb{Z}$ be such that $|Q|=|\operatorname{det} A|^{-j}>|\operatorname{det} A|^{-i}=|P|$. By Lemma 6.4 with $R=M$ for the same choice of $g$ and $h$ as in Case 3, we have

$$
\begin{aligned}
\left|\left\langle\Psi_{P}, \Phi_{Q}\right\rangle\right| & \leq C|\operatorname{det} A|^{-(i-j) / 2}\left(1+|\operatorname{det} A|^{j} \rho_{A}\left(x_{Q}-x_{P}\right)\right)^{-M} \\
& \leq C|\operatorname{det} A|^{-(i-j)(J-\alpha+\epsilon / 2-1 / 2)}\left(1+|\operatorname{det} A|^{j} \rho_{A}\left(x_{Q}-x_{P}\right)\right)^{-J-\epsilon},
\end{aligned}
$$

where $\epsilon / 2=\min (-(J-\alpha-1),(M-J) / 2)>0$.

Combining Cases $1-4$, we conclude that

$$
\begin{aligned}
\left|\left\langle\Psi_{P}, \Phi_{Q}\right\rangle\right| & \leq C\left(1+\frac{\rho_{A}\left(x_{Q}-x_{P}\right)}{\max (|P|,|Q|)}\right)^{-J-\epsilon} \cdot \begin{cases}(|Q| /|P|)^{\alpha+(1+\epsilon) / 2}, & |Q| \leq|P|, \\
(|Q| /|P|)^{\alpha-J+(1-\epsilon) / 2}, & |Q|>|P|,\end{cases} \\
& =C \kappa_{Q P}(\epsilon),
\end{aligned}
$$

which completes the proof of Lemma 5.1

\section{REFERENCES}

[1] K.F. Andersen, R.T. John, Weighted inequalities for vector-valued maximal functions and singular integrals, Studia Math. 69 (1980), 19-31. MR0604351 (82b:42015)

[2] M.Z. Berkola1̌ko, I.Ya. Novikov, Unconditional bases in spaces of functions of anisotropic smoothness, Proc. Steklov Inst. Math. 204 (1994), 27-41. MR.1320017 (96c:46037)

[3] M.Z. Berkolaŭko, I.Ya. Novikov, Wavelet bases and linear operators in anisotropic LizorkinTriebel spaces, Dokl. Akad. Nauk, 340 (1995), 583-586. MR.1327833 (96a:42039)

[4] O.V. Besov, V.P. Il'in, S.M. Nikol'skiı̌, Integral representations of functions and imbedding theorems. Vol. I and II, V. H. Winston \& Sons, Washington, D.C., 1979. MR0519341 (80f:46030a) MR0521808 (80f:46030b)

[5] M. Bownik, A characterization of affine dual frames in $L^{2}\left(\mathbb{R}^{n}\right)$, Appl. Comput. Harmon. Anal. 8 (2000), 203-221. MR.1743536 (2001d:42019)

[6] M. Bownik, Anisotropic Hardy spaces and wavelets, Mem. Amer. Math. Soc. 164 (2003), no. 781, 122 pp. MR1982689 (2004e:42023) 
[7] M. Bownik, Atomic and Molecular Decompositions of Anisotropic Besov Spaces, Math. Z. (to appear).

[8] H.-Q. Bui, Weighted Besov and Triebel spaces: interpolation by the real method, Hiroshima Math. J. 12 (1982), 581-605. MR.0676560 (84f:46038)

[9] H.-Q. Bui, Characterizations of weighted Besov and Triebel-Lizorkin spaces via temperatures, J. Funct. Anal. 55 (1984), 39-62. MR0733032 (86a:46034)

[10] H.-Q. Bui, Weighted Young's inequality and convolution theorems on weighted Besov spaces, Math. Nachr. 170 (1994), 25-37. MR1302364 (95i:46038)

[11] H.-Q. Bui, M. Paluszyński, M.H. Taibleson, A maximal function characterization of weighted Besov-Lipschitz and Triebel-Lizorkin spaces, Studia Math. 119 (1996), 219-246. MR 1397492 (97c:46040)

[12] H.-Q. Bui, M. Paluszyński, M.H. Taibleson, Characterization of the Besov-Lipschitz and Triebel-Lizorkin spaces. The case $q<1$, J. Fourier Anal. Appl. 3 (1997), 837-846. MR $\overline{1600199}$ (99d:46045)

[13] A.P. Calderón, An atomic decomposition of distribution in parabolic $H^{p}$ spaces, Adv. in Math. 25 (1977), 216-225. MR0448066 (56:6376)

[14] A.P. Calderón, A. Torchinsky, Parabolic maximal function associated with a distribution, Adv. in Math. 16 (1975), 1-64. MR0417687 (54:5736)

[15] A.P. Calderón, A. Torchinsky, Parabolic maximal function associated with a distribution II, Adv. in Math. 24 (1977), 101-171. MR0450888 (56:9180)

[16] C. Chui, W. Czaja, M. Maggioni, G. Weiss, Characterization of general tight wavelet frames with matrix dilations and tightness preserving oversampling, J. Fourier Anal. Appl. 8 (2002), no. 2, 173-200. MR 1891728 (2003a:42038)

[17] R.R. Coifman, A real variable characterization of $H^{p}$, Studia Math. 51 (1974), 269-274. MR0358318 (50:10784)

[18] R.R. Coifman, G. Weiss, Analyse harmonique non-commutative sur certains espaces homogenes, Lecture Notes in Math., \#242, Springer-Verlag (1971). MR0499948 (58:17690)

[19] R.R. Coifman, G. Weiss, Extensions of Hardy spaces and their use in analysis, Bull. Amer. Math. Soc. 83 (1977), 569-645. MR0447954 (56:6264)

[20] H. Dappa, W. Trebels, On anisotropic Besov and Bessel potential spaces, Approximation and function spaces (Warsaw, 1986), 69-87, PWN, Warsaw (1989). MR.1097182 (92b:46038)

[21] W. Farkas, Atomic and subatomic decompositions in anisotropic function spaces, Math. Nachr. 209 (2000), 83-113. MR1734360 (2001h:46049)

[22] C. Fefferman, E.M. Stein, Some maximal inequalities, Amer. J. Math. 93 (1971), 107-115. MR0284802 (44:2026)

[23] C. Fefferman, E.M. Stein, $H^{p}$ spaces of several variables, Acta Math. 129 (1972), 137-193. MR0447953 (56:6263)

[24] G.B. Folland, E.M. Stein, Hardy spaces on homogeneous groups, Princeton University Press, Princeton, N.J., 1982. MR0657581 (84h:43027)

[25] M. Frazier, B. Jawerth, Decomposition of Besov spaces, Indiana U. Math. J. 34 (1985), 777-799. MR0808825 (87h:46083)

[26] M. Frazier, B. Jawerth, The $\varphi$-transform and applications to distribution spaces, Lecture Notes in Math., \#1302, Springer-Verlag (1988), 223-246. MR0942271 (89g:46064)

[27] M. Frazier, B. Jawerth, A Discrete Transform and Decomposition of Distribution Spaces, J. Funct. Anal. 93 (1989), 34-170. MR.1070037 (92a:46042)

[28] M. Frazier, B. Jawerth, G. Weiss, Littlewood-Paley Theory and the Study of Function Spaces, CBMS Regional Conference Ser., \#79, American Math. Society (1991). MR 1107300 (92m:42021)

[29] J. García-Cuerva, J.L. Rubio de Francia, Weighted Norm Inequalities and Related Topics, North-Holland (1985). MR.0807149 (87d:42023)

[30] I. Genebashvili, A. Gogatishvili, V. Kokilashvili, M. Krbec, Weight theory for integral transforms on spaces of homogeneous type, Longman, Harlow (1998). MR 1791462 (2003b:42002)

[31] J. Gilbert, Y. Han, J. Hogan, J. Lakey, D. Weiland, G. Weiss, Smooth molecular decompositions of functions and singular integral operators, Mem. Amer. Math. Soc. 156 (2002). MR1880991 (2003f:42026)

[32] K.-P. Ho, Anisotropic Function spaces, Ph.D. Dissertation, Washington University (2002).

[33] P.-G. Lemarié-Rieusset, Projecteurs invariants, matrices de dilatation, ondelettes et analyses multi-résolutions Rev. Mat. Iberoamericana 10 (1994), 283-347. MR1286477(95e:42039) 
[34] Y. Meyer, Wavelets and operators, Cambridge University Press, Cambridge (1992). MR:1228209 (94f:42001)

[35] Y. Meyer, R. Coifman, Wavelets. Calderón-Zygmund and multilinear operators, Cambridge University Press, Cambridge (1997). MR 1456993 (98e:42001)

[36] J. Peetre, New thoughts on Besov spaces, Duke University Mathematics Series, No. 1, Mathematics Department, Duke University, Durham, N.C., 1976. MR0461123 (57:1108)

[37] A. Seeger, A note on Triebel-Lizorkin spaces, Approximation and function spaces (Warsaw, 1986), 391-400, PWN, Warsaw (1989). MR1097208 (92c:46043)

[38] E.M. Stein, Harmonic Analysis: Real-Variable Methods, Orthogonality, and Oscillatory Integrals, Princeton Univ. Press (1993). MR.1232192(95c:42002)

[39] H.-J. Schmeisser, H. Triebel, Topics in Fourier Analysis and Function Spaces, John Wiley \& Sons (1987). MR0891189 (88k:42015b)

[40] J.-O. Strömberg, A. Torchinsky, Weighted Hardy Spaces, Lecture Notes in Math., \#1381, Springer-Verlag (1989). MR1011673 (90j:42053)

[41] W. Szlenk, An introduction to the theory of smooth dynamical systems, Translated from the Polish by Marcin E. Kuczma, PWN-Polish Scientific Publishers, Warsaw (1984). MR:0791919 (86f:58042)

[42] H. Triebel, Theory of Function Spaces, Monographs in Math., \#78, Birkhäuser (1983). MR0781540 (86j:46026)

[43] H. Triebel, Theory of function spaces II, Monographs in Math., \#84, Birkhäuser Verlag, Basel (1992). MR $1163193(93 \mathrm{f}: 46029)$

Department of Mathematics, University of Michigan, 525 East University Ave., Ann Arbor, Michigan 48109

Current address: Department of Mathematics, University of Oregon, Eugene, Oregon 974031222

E-mail address: mbownik@uoregon.edu

Department of Mathematics, Hong Kong University of Science and Technology, Clear Water Bay, Hong Kong (China)

E-mail address: makho@ust.hk 